\title{
Robust multiplicity with a grain of naiveté
}

\author{
Aviad Heifetz \\ Department of Management and Economics, Open University of Israel \\ Willemien Kets \\ Department of Economics, University of Oxford
}

\begin{abstract}
Rationalizability is a central concept in game theory. Since there may be many rationalizable strategies, applications commonly use refinements to obtain sharp predictions. In an important paper, Weinstein and Yildiz (2007) show that no refinement is robust to perturbations of high-order beliefs. We show that robust refinements do exist if we relax the assumption that all players are unlimited in their reasoning ability. In particular, for a class of models, every strict Bayesian-Nash equilibrium is robust. In these environments, a researcher interested in making sharp predictions can use refinements to select among the strict equilibria of the game, and these predictions will be robust.

KEYwords. Robustness, games with incomplete information, rationalizability, finite depth of reasoning, higher-order beliefs, level- $k$ models, global games, refinements.
\end{abstract}

JEL CLASSIFICATION. C72, D8.

\section{INTRODUCTION}

Rationalizability is a fundamental concept in game theory. As it often yields a large set of predictions, it is common for applications to use refinements. Since modeling a strategic situation inherently involves making strong simplifying assumptions that are satisfied only approximately in reality, it is important that any refinement be robust to slight perturbations of the modeling assumptions. In an important paper, Weinstein and Yildiz (2007) show a surprising negative result: if a researcher cannot observe players' actual higher-order beliefs about payoffs (without any error) and there are no restrictions on payoffs, then refinements cannot eliminate any rationalizable strategy. This suggests

Aviad Heifetz: aviadhe@openu. ac.il

Willemien Kets: willemien.kets@economics.ox.ac.uk

This paper supersedes Heifetz and Kets (2012). We are grateful to the editor and two anonymous referees for excellent comments and suggestions that have significantly improved the paper. We thank Sandeep Baliga, Eddie Dekel, Eduardo Faingold, Amanda Friedenberg, Alessandro Pavan, David Pearce, Antonio Penta, Andrés Perea, Phil Reny, Marciano Siniscalchi, and Satoru Takahashi for valuable input, and we are grateful to seminar audiences at Hebrew University, Northwestern University, Tel Aviv University, NYU, Penn State, Queen's University, University of Warwick, and University of Bonn, and conference participants at the SING8 conference (Budapest) and the EEA-ESEM conference (Toulouse) for helpful comments. We thank Luciano Pomatto for excellent research assistance.

Copyright (C) 2018 The Authors. Theoretical Economics. The Econometric Society. Licensed under the Creative Commons Attribution-NonCommercial License 4.0. Available at http: //econtheory . org. 
that if we have only partial knowledge of players' payoff uncertainty, "accounting for incomplete information... casts doubt on all refinements" (Weinstein and Yildiz 2007, p. 367).

This paper challenges this negative conclusion. We show that refinements can be robust if uncertainty about players' reasoning ability is taken into account. Allowing for uncertainty about players' reasoning ability is natural. Experiments suggest that the standard assumption in game theory that players have an infinite depth of reasoningi.e., that they form beliefs about payoffs, about others' beliefs about payoffs, about the others' beliefs about their opponents' beliefs, and so on, ad infinitum-is an idealization at best (Crawford et al. 2013). In many cases, players have a finite depth of reasoning, think that others have a finite depth, or think that others think that their opponent has a finite depth, and so on. Assuming that all types have an infinite depth of reasoning, as standard models do, thus constitutes a strong restriction on beliefs. Accordingly, to test the robustness of predictions, a researcher should consider not only perturbations of beliefs about payoffs, but also about reasoning ability.

Standard models in fact assume not only that players have an infinite depth of reasoning, but also that this is common belief: all players have an infinite depth, believe that others have an infinite depth, etc. ${ }^{1}$ Under this assumption (and a richness assumption on the set of possible payoffs), Weinstein and Yildiz (2007) show that if a type has multiple rationalizable actions, then each of these actions can be made uniquely rationalizable by perturbing the type's belief appropriately. This unique prediction is then robust to further belief perturbations. An important implication is that there are no robust refinements of rationalizability in their setting: if we cannot measure the player's type with infinite precision, then for any of the rationalizable actions, we cannot rule out that this action is uniquely rationalizable for the player. Therefore, if a refinement of rationalizability is robust to alternative specifications of beliefs, then it must select each of the rationalizable actions for the type, and the resulting predictions of the refinement are no stronger than those of rationalizability.

This means that, under the assumption that there is common belief in an infinite depth, there is no scope for refinements when a researcher is concerned with the robustness of his predictions. First, if a type has multiple rationalizable actions, then he cannot robustly select a subset of rationalizable actions. Second, if a type has a unique rationalizable action, then his prediction is robust, but his prediction is determined entirely by mutual beliefs about payoffs. This leaves no room for the researcher to use axiomatic principles (such as payoff dominance) or other criteria (such as those derived from learning or evolutionary models) to further refine his predictions.

This paper challenges both these conclusions. We show that if we depart slightly from standard assumptions and consider environments where players have an infinite depth of reasoning and almost-common belief in an infinite depth, then multiplicity can be robust: there are types with multiple rationalizable actions such that all nearby types have the same rationalizable actions (i.e., the rationalizability correspondence is locally constant at these types). This implies that, unlike in the standard case, mutual

\footnotetext{
${ }^{1}$ See Proposition 2 for a formal statement.
} 
beliefs about payoffs do not necessarily tie down the predictions of a researcher who is concerned with the robustness of his predictions: on the set of types with robust multiplicity, the researcher can select one of the rationalizable actions, and the resulting prediction is robust. This motivates us to study the robustness of one of the most common refinements of rationalizability, (Bayesian-Nash) equilibrium. We show that for a class of environments, every strict equilibrium is robust.

Our results have implications for the conditions under which a researcher can make sharp predictions. As in the standard case with common belief in an infinite depth, a researcher who is concerned with the robustness of his predictions is limited in his ability to make predictions. However, the challenges in both cases are very different. In the standard case, the only predictions of a refinement that retain their validity when the researcher has only partial information about the players' beliefs are those predictions that are true for all rationalizable strategies. This implies that the researcher cannot obtain sharper predictions than those provided by rationalizability unless he is willing to give up robustness. By contrast, if we allow for uncertainty about players' reasoning ability, there need not be a trade-off: robust refinements of rationalizability exist. However, the robustness requirement alone does not select a particular equilibrium beyond the requirement that incentives are strict: in the models that we identify, every strict Bayesian-Nash equilibrium is robust. To select a particular (strict) equilibrium, the researcher will have to appeal to refinements. In this case, refinements are not just consistent with robustness; they are in fact necessary to make sharp predictions.

This paper is the first to study the robustness of predictions under a larger class of belief perturbations than commonly considered. ${ }^{2}$ Unlike the existing robustness literature, which considers only perturbations of beliefs about payoffs, we allow for perturbations of beliefs about both payoffs and reasoning ability. We show that allowing for uncertainty about players' reasoning ability has a significant impact on the continuity properties of the rationalizability correspondence and the robustness of predictions, even if the deviation from standard assumptions is small. This is particularly striking given that the characterization results of Weinstein and Yildiz (2007) have otherwise proven to be extremely robust: they extend to dynamic games (Chen 2012, Weinstein and Yildiz 2013), to games that do not satisfy the richness assumption on payoffs (Penta 2013, Chen et al. 2014a), and to general information structures (Penta 2012). ${ }^{3}$ Our results thus suggest that accounting for uncertainty about reasoning ability can lead to novel insights.

The idea that a "grain" of bounded rationality may affect the behavior of rational players has a long history in game theory. Within this literature, our work is most closely related to Kreps et al. (1982), Kreps and Wilson (1982), and Milgrom and Roberts (1982),

\footnotetext{
${ }^{2}$ In a recent paper, Strzalecki (2014) considers the effect of perturbations of beliefs about reasoning ability in the context of the electronic-mail game of Rubinstein (1989). However, Strzalecki does not study the robustness of predictions; see Section 6.

${ }^{3} \mathrm{~A}$ number of authors have shown that the results of Weinstein and Yildiz (2007) do not necessarily extend to other settings. For example, the results do not extend when the topology is changed (Dekel et al. 2006, Chen et al. 2010, 2017) or if an alternative robustness concept is applied (Chen et al. 2014b). Unlike us, these papers do not consider arbitrarily small deviations from standard assumptions.
} 
who consider the effect of a small amount of doubt about the opponent's rationality. Unlike the irrational types in the existing literature, our nonstrategic types are not committed to taking a certain action. As we discuss in Section 6, this requires novel techniques and leads to new insights.

The next section provides an informal overview of our results and puts them in a broader context. The formal treatment starts in Section 3.

\section{Preview of main results}

\subsection{Framework}

Standard type spaces model players with an infinite depth of reasoning. As suggested by Harsanyi (1967) and shown formally by Mertens and Zamir (1985), each standard type unfolds into a belief hierarchy with an infinite depth that specifies a player's firstorder belief $\mu^{1}$ (i.e., a probability distribution on the payoff parameters), his secondorder belief $\mu^{2}$ (i.e., his belief about the other player's first-order belief), and so on, ad infinitum.

Relaxing this strong assumption requires making the assumptions on players' depth of reasoning explicit within a space of belief hierarchies with an arbitrary (finite or infinite) depth of reasoning. A belief hierarchy has finite depth $k$ if it specifies a player's first-order belief $\mu^{1}$, his second-order belief $\mu^{2}$, and so on, up to his $k$ th-order belief $\mu^{k}$ but no further. The space of all belief hierarchies (with finite or infinite depth) defines the universal type space for players with an arbitrary depth of reasoning, denoted $\mathcal{T}^{*}$. As in the universal type space $\mathcal{T}^{\mathrm{MZ}}$ of Mertens and Zamir (1985) for standard type spaces, every belief hierarchy in $\mathcal{T}^{*}$ defines a type. ${ }^{4}$ With this model in hand, we have the following intuitive characterization.

Proposition 2 (Characterization of standard types). The types from standard type spaces are precisely those types in the universal type space $\mathcal{T}^{*}$ that have an infinite depth of reasoning and that have common belief in the event that players have an infinite depth of reasoning.

This result says that standard types satisfy strong common-knowledge restrictions on their beliefs about players' reasoning ability: not only are players assumed to have an infinite depth of reasoning, they also believe that other players have an infinite depth of reasoning, believe that others believe that, and so on.

Now that these assumptions are made explicit, we can weaken them by considering types in the universal type space that satisfy slighter weaker assumptions. This requires a notion of closeness of beliefs, formally captured by the topology on the type space. The topology reflects what the researcher can learn about the players' types if his observation of their beliefs is imperfect: if a player's actual type is in an open set $O$ and his observation is sufficiently precise, the researcher would conclude that the player's type is in fact in $O$, even if he may never learn the player's true type. We have in mind a researcher who, if he observes a player's beliefs $\mu^{1}, \ldots, \mu^{m}$ up to some finite-order $m$,

\footnotetext{
${ }^{4}$ The converse also holds: every type (with a finite or infinite depth) corresponds to a type in $\mathcal{T}^{*}$; see Appendix B.
} 
then he finds possible any type whose beliefs $\nu^{1}, \ldots, \nu^{m}$ are close to the observed beliefs. On the other hand, he rules out types whose beliefs are very different from the observed beliefs. In particular, he rules out types with a depth of reasoning strictly less than $m .^{5}$

Since applied researchers sometimes restrict attention to a subset of types, we also want our notion of closeness to be independent of the choice of model. For example, if a researcher considers two types to be close when his model contains only types with depth at most $k$, then he should also consider them close if his model also includes types of higher depth. In particular, if the researcher considers two types to be close when his model is given by the universal type space $\mathcal{T}^{\mathrm{MZ}}$ for standard type spaces, then he will also deem them close if his model is the more general model $\mathcal{T}^{*}$ and vice versa.

Together, these two considerations pin down the topology: we use the product topology on the set $H^{k}$ of types with a given depth $k$ of reasoning, and then "glue" the spaces $H^{k}, k \leq \infty$, together using the sum topology. ${ }^{6}$

In this topology, types are close to standard types if they have an infinite depth of reasoning and have $m$ th-order mutual belief in the event that players have an infinite depth of reasoning for some large but finite $m$. That is, types are close to a standard type if they believe (i.e., assign probability 1 to the event) that players have an infinite depth, they believe that players believe that players have an infinite depth, and so on up to the statement that includes the word "believe" $m$ times, but no further. In that case, we say that there is almost-common belief in an infinite depth.

We start by considering interim correlated rationalizability (Dekel et al. 2007). An action is (interim correlated) rationalizable for a type if it survives the iterated elimination of strictly dominated strategies. We assume that a depth-1 type acts as if his opponent is nonstrategic and can play any action. This is in the spirit of the level- $k$ literature, which assumes that a level-1 type plays a best response against a nonstrategic level-0 type that chooses his action uniformly at random (Crawford et al. 2013).

A researcher who cannot measure players' belief hierarchies with infinite precision may want his prediction to be robust against small perturbations. To capture this, say that a subset $A_{i}^{\prime}$ of actions for player $i$ is robustly rationalizable for a type $h_{i}$ if, when the player's actual type is $h_{i}$, then the researcher would conclude that $i$ 's rationalizable actions are precisely the actions in $A_{i}^{\prime}$ whenever he can measure $i$ 's belief with sufficient (but finite) precision. As the topology reflects what a researcher can learn about the players' types, this is the case precisely if there is a neighborhood of $h_{i}$ (i.e., an open subset $O\left(h_{i}\right)$ that contains $\left.h_{i}\right)$ such that the set of rationalizable actions is $A_{i}^{\prime}$ across all types in the neighborhood.

\subsection{Robust multiplicity}

Since every game-theoretic model is an idealization of the true strategic environment, an important question is whether predictions are robust to relaxing strong assumptions embodied in the model. The case of complete-information games has received

\footnotetext{
${ }^{5}$ In particular, a type with finite depth $m$ is not close to a type with depth $m-1$, even if the types have the same beliefs up to order $m-1$.

${ }^{6}$ The sum topology preserves the open sets in the component spaces without adding extraneous open sets: it is the weakest (i.e., smallest) topology that contains the open sets in $H^{k}, k \leq \infty$.
} 
particular attention in the literature. Complete-information models are an idealization of situations where payoffs are observed only with some noise. The predictions of complete-information models may not be robust to the introduction of a small amount of incomplete information, as the following familiar example illustrates.

EXAMPLE 1. Consider the following payoff matrix, taken from Carlsson and van Damme (1993):

\begin{tabular}{c|c|c|}
\multicolumn{1}{c}{} & \multicolumn{1}{c}{$I$} & \multicolumn{1}{c}{$N I$} \\
\cline { 2 - 3 }$I$ & $\theta, \theta$ & $\theta-1,0$ \\
\cline { 2 - 3 }$N I$ & $0, \theta-1$ & 0,0 \\
\cline { 2 - 3 } & &
\end{tabular}

The state $\theta$ is drawn uniformly at random from [-1,2]. Players can choose to invest (i.e., play $I$ ) or to not invest (i.e., play $N I$ ). Each player $i$ receives a (potentially noisy) signal $x_{i}$ about the state: if the state is $\theta$, then each player receives a signal $x_{i}$ drawn uniformly at random from $[\theta-\varepsilon, \theta+\varepsilon]$, independently across players, where $\varepsilon \geq 0$ is small (say, $\varepsilon<\frac{1}{2}$ ). Hence, players' observations of $\theta$ become increasingly precise as $\varepsilon$ approaches 0 . If there is complete information about payoffs (i.e., $\varepsilon=0$ ), then both actions are rationalizable for any signal $x_{i} \in(0,1)$.

This multiplicity may not be robust, however. Assuming that players have an infinite depth of reasoning and this is common belief, Carlsson and van Damme (1993) show that the risk-dominant equilibrium is the unique prediction if the noise is small (i.e., $\varepsilon$ positive but close to 0 ). So, in the standard model, the prediction that both actions are rationalizable is not robust to relaxing the assumption that $\varepsilon=0$.

There is thus a striking discontinuity between the case where the payoffs are common belief (i.e., $\varepsilon=0$ ) and where they are almost-common belief (i.e., $\varepsilon>0$ ), at least if players have an infinite depth of reasoning and this is common belief. This type of sensitivity is general.

Proposition 3 (No robust multiplicity with common belief in an infinite depth (Weinstein and Yildiz 2007, Proposition 2)). If the set of possible payoff functions is sufficiently rich, then robust multiplicity is not consistent with common belief in an infinite depth of reasoning. That is, there there are no types with multiple rationalizable actions such that nearby types have the same rationalizable actions.

The result says the following: Suppose a player's actual type has an infinite depth and has common belief in an infinite depth, and the researcher observes the beliefs of the type up to some finite (but potentially very high) order. If he finds that multiple rationalizable actions are consistent with his observation, then he cannot rule out that one of these actions will turn out to be the unique rationalizable action if he were to observe more orders of beliefs. In other words, under the assumption that types have an infinite depth and common belief in an infinite depth, a researcher cannot conclude that a type has multiple rationalizable actions unless he can observe the full hierarchy of beliefs. For instance, in Example 1, if the researcher does not want to impose strong common-knowledge restrictions (i.e., $\varepsilon=0$ ), then under the assumption that types have an infinite depth and common belief in an infinite depth, he cannot conclude that a type 
with signal $x_{i}=\frac{1}{4}$ (say) has multiple rationalizable actions if he can observe only finitely many orders of beliefs, even if he is confident that $\varepsilon$ is close to 0 .

Weinstein and Yildiz's result holds very generally. Thus, there seems to be no hope to have robust multiplicity in a standard type space unless one is willing to make commonknowledge assumptions on the payoff functions. However, by working with standard type spaces, Weinstein and Yildiz do make the strong assumption that players have an infinite depth of reasoning and have common belief in an infinite depth. Our first main result shows that multiplicity can be robust when this strong assumption is relaxed.

Proposition 4 (Robust multiplicity with almost-common belief in an infinite depth). If the set of possible payoff functions is sufficiently rich, then robust multiplicity is consistent with an infinite depth of reasoning and almost-common belief in an infinite depth. That $i$ s, given a set $A^{\prime}$ of actions with $\left|A^{\prime}\right|>1$, there exist types $h^{m}, m=1,2, \ldots$, with an infinite depth of reasoning and mth-order mutual belief in an infinite depth for whom $A^{\prime}$ is robustly rationalizable.

Thus, while the existing literature shows that perturbing players' beliefs about payoffs can give unique predictions (e.g., Rubinstein 1989, Carlsson and van Damme 1993, Weinstein and Yildiz 2007), Proposition 4 shows that by perturbing beliefs about reasoning ability, we can obtain robust multiplicity. We explain the intuition behind Proposition 4 using a variant of Example 1.

Example 2. Players believe that the state $\theta$ is either $\theta=2$ or $\theta=-1$. That is, the possible payoff matrices are

\begin{tabular}{c|c|c|}
\multicolumn{1}{c}{} & \multicolumn{1}{c}{$I$} & $N I$ \\
\cline { 2 - 3 }$I$ & 2,2 & 1,0 \\
\cline { 2 - 3 }$N I$ & 0,1 & 0,0 \\
\cline { 2 - 3 } & \multicolumn{2}{c}{$\theta=2$}
\end{tabular}

\begin{tabular}{c|c|c|}
\multicolumn{1}{c}{$I$} & \multicolumn{1}{c}{$N I$} \\
\cline { 2 - 3 }$I$ & $-1,-1$ & $-2,0$ \\
\cline { 2 - 3 }$N I$ & $0,-2$ & 0,0 \\
\cline { 2 - 3 } & \multicolumn{2}{c}{$\theta=-1$}
\end{tabular}

In this case, investing is a strict best response for any player who assigns probability $p>\frac{2}{3}$ to $\theta=2$, and not investing is a strict best response for a player if he assigns probability $p<\frac{1}{3}$ to $\theta=2$. If $p \in\left(\frac{1}{3}, \frac{2}{3}\right)$, then either action is a strict best response for a player depending on his conjecture about the opponent's behavior: under the conjecture that the opponent invests, investing is the unique best response; and under the conjecture that the opponent does not invest, not investing is the unique best response. The probability distributions that assign probability $p \in\left(\frac{1}{3}, \frac{2}{3}\right)$ to $\theta=2$ define what we call a multiplicity set.

To show that multiplicity can be robust for a type with almost-common belief in an infinite depth, we start with a "grain" of robust multiplicity and use a contagion argument to show that multiplicity is robust for types with almost-common belief in an infinite depth.

The grain consists of finite-depth types. We start with the depth-1 types. Depth-1 types form beliefs only about the payoff parameter $\theta$ and act as if their opponent can play any action. Both actions are rationalizable for a depth-1 type $h^{1}$ with a belief in the multiplicity set (i.e., that assign probability $p \in\left(\frac{1}{3}, \frac{2}{3}\right)$ to $\left.\theta=2\right)$, and the same is true for 
depth-1 types with beliefs sufficiently close to $h^{1}$. So both actions are robustly rationalizable for type $h^{1}$. Then, by a similar argument, both actions are robustly rationalizable for a depth-2 type $h^{2}$ with a belief in the multiplicity set that believes that the opponent's type is $h^{1}$ or some nearby type for whom both actions are rationalizable. We can iterate this argument to show that for any $k=1,2, \ldots$, there is a depth- $k$ type $h^{k}$ for whom both actions are robustly rationalizable.

This allows us to show that multiplicity can be robust under almost-common belief in an infinite depth. Consider a type with an infinite depth with a belief in the multiplicity set that believes that the opponent has a finite-depth type for whom both actions are robustly rationalizable. By a similar argument as before, both actions are robustly rationalizable for the type. Again, by iterating the argument, we can show that for any $m=1,2, \ldots$, there are infinite-depth types with $m$ th-order mutual belief in the event that players have an infinite depth for whom both actions are robustly rationalizable. Hence, multiplicity can be robust under almost-common belief in an infinite depth.

This example illustrates the key difference between the standard framework and the more general framework considered here: when players can have an arbitrary depth of reasoning, there is a grain of robust multiplicity formed by types with a finite depth of reasoning. The intuition is straightforward. Suppose that a player's actual type has finite depth $k$. If the researcher finds that multiple actions can be (strictly) rationalizable given his observation, then he can rule out that the type has a unique rationalizable action by making sufficiently precise observations of the type's beliefs up to order $k+1$ : by doing so, he can rule out that the type has beliefs at orders greater than $k$, and if his observations of the type's beliefs up to $k$ are sufficiently precise, then he will learn the type's rationalizable actions. Thus, he can be confident that his predictions are not sensitive to the precise specification of beliefs at arbitrarily high orders.

Somewhat surprisingly, the robustness of multiplicity extends well beyond types with a finite depth of reasoning: once a grain of robust multiplicity has been identified, a contagion argument can be used to establish the robustness of multiplicity for types that are arbitrarily close to standard types, that is, to types with an infinite depth and high-order mutual belief in an infinite depth. The intuition is subtle, so a full discussion is deferred to Section 4. However, a key insight is that if there is a grain of robust multiplicity, then even if a player's actual type is close to a standard type, a researcher who observes beliefs up to some finite order $m$ can rule out that the type's rationalizable actions depend sensitively on its belief at orders greater than $m$ if he finds that the type assigns only low probability to types with depth at least $m$, or to types that assign high probability to types with a high depth, or to types that assign high probability to the other player assigning high probability to such types, and so on.

The proof method thus bears some similarities with the proofs in the existing robustness literature, which "[depend] critically on the existence... of a subclass of dominance solvable games that serve as take-offs for the iterated dominance argument, and, thus, exert a kind of remote influence on the games with multiple equilibria" (Carlsson and van Damme 1993, p. 992). Proposition 4 depends on the existence of a grain of types 
with robust multiplicity that form the starting point of a contagion argument that establishes the robustness of multiplicity for other types. The critical difference is that our grain and our contagion argument involve multiplicity, not uniqueness and dominancesolvability as in the existing literature; also see Section 6.

Proposition 4 has implications for the type of observations that allow a researcher to conclude that his prediction is valid for all models consistent with his observations if he cannot observe the full hierarchy of beliefs. If there is a unique action that is rationalizable given a researcher's observation, then he can be confident that his prediction is robust. Intuitively, observing more orders of beliefs can only eliminate actions from the set of rationalizable actions consistent with the observations, not add new ones. ${ }^{7}$ Alternatively, if the player's actual type has multiple rationalizable actions, then, under the assumption that there is common belief in an infinite depth, a researcher cannot rule out that the type has a unique rationalizable action unless he observes the player's entire hierarchy of beliefs (Proposition 3). Proposition 4 shows that if we relax the assumption that there is common belief in an infinite depth, then this need not be the case. If, by observing sufficiently many orders of beliefs, the researcher learns that the type's depth is finite and multiple actions can be strictly rationalizable given his observation, then, by making sufficiently precise observations of the type's finite belief hierarchy, he can learn the set of rationalizable actions for the type, as in Example 2. But the researcher can make robust predictions even if he does not learn that the type's depth is bounded. For example, if by observing the type's $k$ th-order beliefs for increasing (but finite) $k$, the researcher learns that the type assigns a vanishingly small probability to the event that the other player continues to reason beyond order $k-1$, then he can be confident that his prediction will not be sensitive to the type's beliefs beyond order $k$. Likewise, if the researcher learns that the type assigns low probability to the other player assigning high probability to her opponent continuing to reason, and so on, then his prediction will not be sensitive to the type's (unobserved) beliefs at higher orders.

While Proposition 4 shows that robust multiplicity is consistent with almostcommon belief in an infinite depth in a wide range of situations, it does not speak directly to the discontinuity of behavior in Example 1 as it leaves open the possibility that the types with robust multiplicity have very different beliefs about payoffs than the types in the example. However, multiplicity can be robust also for types that have beliefs about payoffs that are consistent with the information structure in the example. Section 4.2 considers a model $M^{\varepsilon}$ with types whose beliefs are consistent with the information structure in Example 1 and shows the following result.

Proposition 5 (Robust multiplicity around complete-information types). For every $m=$ $1,2, \ldots$, there is an interval $\left(\underline{x}_{m}^{\varepsilon}, \bar{x}_{m}^{\varepsilon}\right) \supsetneq\left\{\frac{1}{2}\right\}$ such that both actions are robustly rationalizable for every infinite-depth type in the model $M^{\varepsilon}$ that has signal $x_{i} \in\left(\underline{x}_{m}^{\varepsilon}, \bar{x}_{m}^{\varepsilon}\right)$ and mth-order mutual belief in an infinite depth whenever $\varepsilon$ is sufficiently small. Moreover, $\underline{x}_{m}^{\varepsilon} \rightarrow 0$ and $\bar{x}_{m}^{\varepsilon} \rightarrow 1$ as $\varepsilon \rightarrow 0$.

${ }^{7}$ For a proof for the standard case, see Dekel et al. (2007); for the case where players can have an arbitrary depth of reasoning, see Corollary 1. 
Proposition 5 says that as the noise level $\varepsilon$ goes to 0 , both actions are rationalizable for any type in $M^{\varepsilon}$ that has a signal $x_{i} \in(0,1)$ and high-order mutual belief in an infinite depth. Thus, the discontinuity in behavior in Example 1 is not robust to relaxing the assumption that there is common belief in an infinite depth. In particular, the riskdominant equilibrium selection of Carlsson and van Damme (1993) does not extend if we relax the assumption that there is common belief in an infinite depth. Proposition 5 thus complements the existing literature: while the literature has shown that the risk-dominant selection is not robust to perturbations of the information structure, the present result shows that the risk-dominant selection is not robust even if we keep the information structure fixed if we allow for perturbations of beliefs about players' reasoning abilities. ${ }^{8}$

Remark 1. Thus far, we have not specified the richness requirement on the set of possible payoff functions. As we discuss, there are different richness assumptions that may be of interest (Assumptions R-Dom and R-Mult $\left(A^{\prime}\right)$ below). The interesting case is when the set of possible payoff functions is rich in both senses. This is the case in all examples as well as in the main applications in the literature (Morris and Shin 2003).

\subsection{Robust refinements}

An important implication of the lack of robust multiplicity in the standard case is that there is no scope for robust refinements if there is common belief in an infinite depth. For example, suppose the payoff matrix is as in Example 1 and $\theta \in\left(0, \frac{1}{2}\right)$ is commonly known (i.e., $\varepsilon=0$ ). Then both actions are rationalizable, and a researcher who subscribes to payoff dominance may want to select the equilibrium in which both players invest. But, by the results of Carlsson and van Damme (1993), this prediction is not robust: if we introduce a small amount of uncertainty about payoffs, then the not-invest equilibrium is uniquely selected. By the results of Weinstein and Yildiz (2007), the prediction that both players will not invest is also not robust: if we perturb beliefs a little, then the unique prediction is that both players invest. Weinstein and Yildiz (2007) show that this holds very generally: if there is common belief in an infinite depth, then a prediction of a refinement is robust if and only if it is true for all rationalizable strategies. Therefore, a researcher cannot make a prediction that is stronger than what is implied by rationalizability in this case.

If we relax the strong assumption that there is common belief in an infinite depth, then multiplicity can be robust, suggesting that there is some scope for robust refinements. To see this, suppose that $\varepsilon$ is close to 0 and that the researcher thinks that the players' beliefs are described by the model $M^{\varepsilon}$ in Proposition 5. Suppose a researcher wants to select the payoff-dominant action whenever it is consistent with rationalizability. Then he could use a refinement of rationalizability that selects the action "invest" for a type whenever it is rationalizable, and coincides with rationalizability otherwise. This

\footnotetext{
${ }^{8}$ See Strzalecki (2014) for a similar result in the context of the electronic-mail game of Rubinstein (1989). However, Strzalecki does not show that the resulting predictions are robust to further belief perturbations.
} 
refinement predicts that every type in $M^{\varepsilon}$ with signal $x_{i} \in(0,1)$ whose observation is sufficiently precise (i.e., $\varepsilon$ close to 0 ) will invest. By Proposition 5, this selection is robust: even if the researcher has misspecified players' beliefs about payoffs or about the opponent's level of sophistication, he can still be confident that players are willing to invest if his assumptions are satisfied approximately. Alternatively, the researcher may want to select the action "not invest" for a type whenever it is rationalizable (and coincides with rationalizability otherwise). Again, this is a proper refinement of rationalizability, and it is robust to perturbations of beliefs both about payoffs and about players' depth of reasoning.

This motivates us to ask whether standard refinements of rationalizability can be robust. We focus on the robustness of Bayesian-Nash equilibrium, one of the most common refinements of rationalizability. Our equilibrium definition is standard: a strategy profile is a (Bayesian-Nash) equilibrium for a model if each type in the model plays a best response to the opponent's strategy. However, we apply the concepts to richer type spaces by allowing players to have a finite depth of reasoning. As before, we assume that a depth-1 type plays as if his opponent is nonstrategic and can choose any action, in line with the level- $k$ literature (Crawford et al. 2013).

Again, we take the perspective of a researcher who can observe finitely many orders of beliefs with some noise: there is some finite order $\kappa$ and some $\eta>0$ such that if a player's actual type is $h_{i}$, then the researcher cannot rule out any type whose $m$ th-order beliefs are $\eta$-close to those of $h_{i}$ for $m \leq \kappa$, where our notion of $\eta$-closeness is determined by the usual weak topology on the set of $m$ th-order beliefs. ${ }^{9}$ This leads to the following robustness notion: a Bayesian-Nash equilibrium $\sigma$ for a model is robust if for some $\eta>0$ and $\kappa<\infty$, every model that the researcher cannot rule out on the basis of his observations (given $\eta$ and $\kappa$ ) has an equilibrium $\sigma^{\prime}$ such that types that are close to the original model play the same actions as under $\sigma$.

The next result shows that for some models, every strict Bayesian-Nash equilibrium is robust.

Proposition 7 (Strict equilibrium robust under almost-common belief in infinite depth). If the set of possible payoff functions is sufficiently rich, then there exist models consistent with an infinite depth of reasoning and almost-common belief in an infinite depth for which every strict Bayesian-Nash equilibrium is robust. That is, for every $m=1,2, \ldots$, there is a model with mth-order mutual belief in an infinite depth for which every strict BayesianNash equilibrium is robust.

As we discuss, the models in Proposition 7 can be chosen in such a way that they include types with multiple rationalizable actions. Hence, an immediate implication of Proposition 7 is that robust (and proper) refinements of rationalizability exist if we relax the assumption that there is common belief in an infinite depth:

Corollary (Robust refinements under almost-common belief in infinite depth). If the set of possible payoff functions is sufficiently rich, then for every $m=1,2, \ldots$, there is a model with mth-order belief in an infinite depth for which there is a robust refinement of rationalizability.

\footnotetext{
${ }^{9}$ Recall that $m$ th-order beliefs are probability distributions, so a sequence $\left\{\mu^{m, n}\right\}_{n}$ of $m$ th-order beliefs converges to an $m$ th-order belief $\mu^{m}$ in the weak topology if it converges in distribution.
} 
Proposition 7 is consistent with a folk result for complete-information games: in environments where payoffs are commonly known among the players, but the researcher is unsure about the payoffs, every strict Nash equilibrium is robust to small misspecifications of the payoffs. Proposition 7 shows that this result extends to games with incomplete information with a suitable form of uncertainty about reasoning ability.

We illustrate the intuition behind Proposition 7 using Example 1. Suppose that a researcher thinks that there is complete information about payoffs (i.e., $\varepsilon=0$ ) and that $\theta \in\left(0, \frac{1}{2}\right)$. However, he recognizes his model may be misspecified, so he would like his prediction to be robust. If his model of players' reasoning ability is as in Proposition 7, then, for the depth-1 types, who act as if they play against a nonstrategic type, he can select either action. If all depth-1 types invest, then it is a strict best response for all depth2 types to invest. A simple inductive argument then shows that there is a Bayesian-Nash equilibrium $\sigma^{I}$ for the model under which all types invest. Likewise, we can construct a strict Nash equilibrium $\sigma^{N I}$ under which no type invests. The former Nash equilibrium is payoff dominant, and the latter is risk dominant. Since all incentives are strict, both predictions are robust: even if we perturb beliefs a little, each type has a unique best response under either strategy. In particular, these predictions are robust to the introduction of a small amount of incomplete information about payoffs.

Both in the standard case with common belief in an infinite depth and in the case where there is almost-common belief in an infinite depth, the researcher is thus limited in his ability to make predictions. However, the difficulties he faces are fundamentally different in the two cases. If there is common belief in an infinite depth, the only robust predictions that a researcher can make are the predictions that are true for all rationalizable actions. In particular, equilibrium cannot refine rationalizability if predictions are required to be robust (Weinstein and Yildiz 2007; also see Proposition 6 below). By contrast, if there is almost-common belief in an infinite depth, then robust refinements of rationalizability do exist for a class of models. However, the requirement that predictions be robust does not select a particular equilibrium; rather, every strict equilibrium is robust in this case (Proposition 7). In particular, in complete-information games with multiple strict Nash equilibria, all strict Nash equilibria are robust to the introduction of a small amount of incomplete information about payoffs in models with a grain of robust multiplicity.

This yields radically different conclusions regarding the scope for the refinement program. Weinstein and Yildiz (2007) argue that since there are no robust refinements of rationalizability when there is common belief in an infinite depth, there is limited or no scope for a refinement program unless a researcher is willing to impose strong commonknowledge restrictions on beliefs (e.g., pp. 374-375). In contrast, the present results suggest that there need not be a tension between robustness and refinements if the strong assumption that there is common belief in an infinite depth is relaxed: by using richer type spaces that specify beliefs not only about payoffs but also about players' reasoning ability, we can extend existing solution concepts and use refinements of these concepts to obtain sharp and robust predictions within this richer context. Our approach thus bears some similarities with that of Carlsson and van Damme (1993), who show that sharp and robust predictions can be obtained by introducing uncertainty about payoffs. But robustness alone does not select an equilibrium beyond the criterion that incentives 


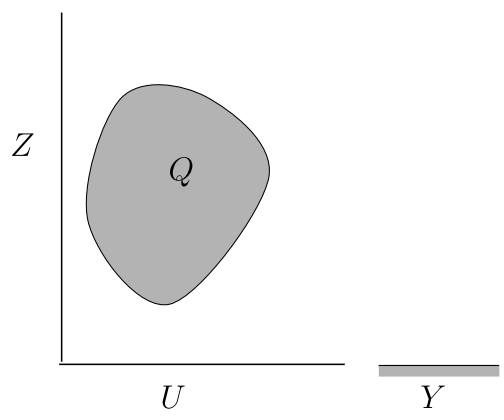

Figure 1. The space $W$ (shaded gray) is the union of $Q \subseteq U \times Z$ and of $Y$. The space $V$ is the union of $U$ and $Y$.

be strict: in models with a grain of robust multiplicity, a researcher who wants to make sharp predictions needs to appeal to refinements to provide sharp predictions.

The remainder of this paper is organized as follows. Section 3 introduces the framework. Section 4 presents our results on robust multiplicity in the context of rationalizability, and Section 5 presents our results on robust refinements of rationalizability. Section 6 discusses the related literature. Proofs and additional results can be found in the Appendices.

\section{Framework}

\subsection{Preliminaries}

We follow the standard conventions for subspaces, products, and (disjoint) unions of topological spaces. That is, a subspace of a topological space is endowed with the relative topology, the product of a collection of topological spaces is endowed with the product topology, and if $\left(V_{\lambda}\right)_{\lambda \in \Lambda}$ is a family of disjoint topological spaces, then $\bigcup_{\lambda} V_{\lambda}$ is endowed with the sum topology, that is, a subset $U \subseteq \bigcup_{\lambda \in \Lambda} V_{\lambda}$ is open in $\bigcup_{\lambda \in \Lambda} V_{\lambda}$ if and only if $U \cap V_{\lambda}$ is open in $V_{\lambda}$ for each $\lambda \in \Lambda .{ }^{10}$

Given a topological space $V$, the set of probability measures on the Borel $\sigma$-algebra $\mathcal{B}(V)$ is denoted by $\Delta(V)$. We endow $\Delta(V)$ with the topology of weak convergence. We extend the definition of a marginal to a union of measurable spaces. Let $V$ be the union of the disjoint sets $U$ and $Y$, and let $Q \subseteq U \times Z$ and $W=Q \cup Y$, where all spaces are assumed to be topological spaces; see Figure 1. Then for $\mu \in \Delta(W)$, denote by $\operatorname{marg}_{V} \mu \in$ $\Delta(V)$ the probability measure defined by

$$
\operatorname{marg}_{V} \mu(E)=\mu(\{(u, z) \in Q: u \in E\})+\mu(E \cap Y)
$$

for every measurable set $E \subseteq V$. This definition reduces to the standard one if $Y$ is empty. If $\mu$ is a probability measure on a product space $U \times Y$, and $E$ is a measurable subset of $U$, then we sometimes write $\mu(E)$ for $\operatorname{marg}_{U} \mu(E)$.

\footnotetext{
${ }^{10}$ As is standard, the Cartesian product of a collection of topological spaces $\left(V_{\lambda}\right)_{\lambda \in \Lambda}$ is denoted by $V$, with typical element $v$. Given $\lambda \in \Lambda$, we write $V_{-\lambda}$ for $\prod_{\ell \in \Lambda \backslash\{\lambda\}} V_{\ell}$, with typical element $v_{-\lambda}$. Likewise, given a family $g_{\lambda}: Y_{\lambda} \rightarrow Z_{\lambda}$ of functions, we write $g(y)$ and $g_{-\lambda}\left(y_{-\lambda}\right)$ for $\left(g_{\lambda}\left(y_{\lambda}\right)\right)_{\lambda \in \Lambda}$ and $\left(g_{\lambda^{\prime}}\left(y_{\lambda^{\prime}}\right)\right)_{\lambda^{\prime} \neq \lambda}$, respectively.
} 


\subsection{Strategic environment}

There are two players, labeled $i=1,2$. The set of states of nature is $\Theta$. Each player $i$ has a set $A_{i}$ of actions and a utility function $u_{i}: A \times \Theta \rightarrow \mathbb{R}$. Players may have private information: each player $i$ has a (payoff-irrelevant) signal $x_{i} \in X_{i}$. We assume that $\Theta$ and $X_{i}$ are compact metric. Action sets are assumed to be finite, and payoff functions are taken to be continuous. The extension to an arbitrary (finite) number of players is straightforward.

We focus on the case where the set of possible payoff functions is sufficiently rich. One such richness requirement, due to Weinstein and Yildiz (2007), is that each action is strictly dominant for some state of nature.

Assumption R-Dom (Richness-dominance (Weinstein and Yildiz 2007, Assumption 1)). For each player $i=1,2$ and each action $a_{i} \in A_{i}$, there is a state $\theta^{a_{i}} \in \Theta$ of nature such that

$$
u_{i}\left(a_{i}, a_{-i}, \theta^{a_{i}}\right)>u_{i}\left(a_{i}^{\prime}, a_{-i}, \theta^{a_{i}}\right)
$$

for all $a_{i}^{\prime} \neq a_{i}$ and $a_{-i} \in A_{-i}$.

An alternative richness condition is that beliefs about payoffs do not fully determine play. That is, for some beliefs about nature, a player can have multiple (strict) best responses, depending on his conjecture about the play of his opponent.

Assumption R-Mult $\left(A^{\prime}\right)$ (Richness multiplicity). Given a product set $A^{\prime} \subset A$ with $\left|A_{i}^{\prime}\right|>1$ for all $i$, for each player $i=1,2$, there is a belief $\mu_{i} \in \Delta(\Theta)$ such that the following statements hold:

(i) For each $a_{i} \in A_{i}^{\prime}$, there is a measurable function $\widetilde{s}_{-i}^{a_{i}}: \Theta \rightarrow \Delta\left(A_{-i}^{\prime}\right)$ such that

$$
\int_{\Theta} u_{i}\left(a_{i}, \widetilde{s}_{-i}^{a_{i}}(\theta), \theta\right) \mathrm{d} \mu_{i}(\theta)>\int_{\Theta} u_{i}\left(a_{i}^{\prime}, \widetilde{s}_{-i}^{a_{i}}(\theta), \theta\right) \mathrm{d} \mu_{i}(\theta) \quad \text { for } a_{i}^{\prime} \neq a_{i} .
$$

(ii) If $a_{i}^{\prime} \notin A_{i}^{\prime}$, then there is no measurable function $\widetilde{s}_{-i}^{a_{i}^{\prime}}: \Theta \rightarrow \Delta\left(A_{-i}\right)$ such that

$$
\int_{\Theta} u_{i}\left(a_{i}^{\prime}, \widetilde{S}_{-i}^{\prime}(\theta), \theta\right) \mathrm{d} \mu_{i}(\theta) \geq \int_{\Theta} u_{i}\left(a_{i}^{\prime \prime}, \widetilde{s}_{-i}^{\prime}(\theta), \theta\right) \mathrm{d} \mu_{i}(\theta) \quad \text { for } a_{i}^{\prime \prime} \neq a_{i}^{\prime} .
$$

The set of such beliefs $\mu$ is denoted by $\Delta_{i}^{A^{\prime}}$.

In words, if Assumption R-Mult $\left(A^{\prime}\right)$ is satisfied for a product set $A^{\prime}$, then each player $i$ has a first-order belief $\mu_{i}$ about payoffs such that any action in $A_{i}^{\prime}$ is a strict best response against some conjecture that the opponent plays an action in $A_{-i}^{\prime}$ and these are the only best responses. ${ }^{11}$

\footnotetext{
${ }^{11}$ While Assumption R-Mult $\left(A^{\prime}\right)$ is sufficient for our results, we conjecture it is not necessary, just like Assumption R-Dom is not necessary for Weinstein and Yildiz's (2007) results (Penta 2013).
} 
Both richness conditions are satisfied if the set of possible payoff functions is sufficiently rich. For example, if the set of possible payoff functions includes all functions (i.e., $\Theta:=[0,1]^{A} \times[0,1]^{A}$ and $u_{i}(a, \theta):=\theta_{i}(a)$ ), then both conditions are satisfied. Global games (e.g., Example 1 and Section 5 ) also satisfy both conditions (with $\left.A^{\prime}=A\right){ }^{12}$

\subsection{Beliefs}

We are interested in how higher-order beliefs impact strategic behavior. Higher-order beliefs can be modeled using belief hierarchies, where a belief hierarchy for player $i$ specifies his belief about the state of nature and the other players' signals (i.e., about $\Theta \times X_{-i}$ ), his beliefs about his opponent's beliefs, and so on, up to some order. ${ }^{13} \mathrm{We}$ allow for an arbitrary depth of reasoning: a belief hierarchy can have any finite or infinite depth. To consider all possible specifications of players' higher-order beliefs, we construct the space of all belief hierarchies. To that aim, we construct two sequences of spaces for each player $i, H_{i}^{m}$ and $\widetilde{H}_{i}^{m}, m \geq 0$, with $H_{i}^{m}$ the set of $m$ th-order belief hierarchies that "stop" reasoning at order $m$, and $\widetilde{H}_{i}^{m}$ the set of belief hierarchies that "continue" to reason at that order. The belief hierarchies in $H_{i}^{m}$ are precisely the belief hierarchies that have depth $m$, while the belief hierarchies in $\widetilde{H}_{i}^{m}$ are used to construct the belief hierarchies that have depth at least $m+1$ (possibly infinite).

It will be convenient to fix two (arbitrary) labels $h_{i}^{* 0}$ and $\tilde{\mu}_{i}^{0}$. The label $h_{i}^{*, 0}$ is similar to the level-0 type in the level- $k$ literature. The label $\tilde{\mu}_{i}^{0}$ is a notational placeholder that will be used to construct other types. Let $\widetilde{H}_{i}^{0}:=X_{i} \times\left\{\tilde{\mu}_{i}^{0}\right\}$ and $H_{i}^{0}:=X_{i} \times\left\{h_{i}^{*, 0}\right\}$ be the set of zeroth-order belief hierarchies that continue and that stop at order 0 , respectively. ${ }^{14}$

We next consider players' beliefs about the state of nature and about whether the other players have stopped reasoning at order 0 . Let

$$
\begin{aligned}
& \widetilde{\Omega}_{i}^{0}:=\Theta \times\left(\widetilde{H}_{-i}^{0} \cup H_{-i}^{0}\right), \\
& \Omega_{i}^{0}:=\Theta \times H_{-i}^{0} .
\end{aligned}
$$

Define the set of first-order belief hierarchies that continue and stop at order 1 by

$$
\begin{aligned}
\widetilde{H}_{i}^{1} & :=\widetilde{H}_{i}^{0} \times \Delta\left(\widetilde{\Omega}_{i}^{0}\right), \\
H_{i}^{1} & :=\widetilde{H}_{i}^{0} \times \Delta\left(\Omega_{i}^{0}\right),
\end{aligned}
$$

${ }^{12}$ The two richness conditions are independent: Assumption R-Mult $\left(A^{\prime}\right)$ does not imply Assumption RDom or vice versa. For example, a complete-information game (i.e., $\Theta=\{\theta\}$ ) with multiple strict equilibria obviously satisfies R-Mult $\left(A^{\prime}\right)$ but does not satisfy R-Dom. A simple example that satisfies R-Dom but not $\operatorname{R-Mult}\left(A^{\prime}\right)$ is one where there are two states, $\theta_{1}, \theta_{2}$, and two actions, $a_{i}^{1}, a_{i}^{2}$, for each player, where each player $i$ receives 1 if he plays $a_{i}^{\ell}$ in $\theta_{\ell}$ and 0 otherwise.

${ }^{13}$ We thus distinguish between a player's private information (i.e., signal) and his belief hierarchy/type, as is common in the literature on the robustness of game-theoretic predictions (e.g., Bergemann and Morris 2005).

${ }^{14}$ The types in $\widetilde{H}_{i}^{0}$ and $H_{i}^{0}$ are introduced merely for notational convenience. Alternatively, we could have started with two copies of $\Delta(\Theta)$ : one to describe the first-order beliefs of depth-1 types, and one to describe the first-order beliefs of types that have depth greater than 1 . 
respectively. These equations describe the first-order beliefs for belief hierarchies that reason beyond the first order and that stop reasoning at the first order, respectively (where a first-order belief describes a player's belief about the state of nature and other player's signal). The first-order belief hierarchies in $\widetilde{H}_{i}^{1}$ will be used to define types of depth greater than 1 , while the first-order belief hierarchies in $H_{i}^{1}$ define the depth-1 types. Types in $\widetilde{H}_{i}^{1}$ thus think it is possible that the other player has not yet stopped reasoning; this will allow us to model that a type of depth $k>1$ thinks that the opponent has depth $m$ at least 1 .

We now define inductively the sets of higher-order belief hierarchies. For $k=$ $1,2, \ldots$, suppose that for each player $j$ and all $\ell \leq k, \widetilde{H}_{j}^{\ell}$ and $H_{j}^{\ell}$ are the sets of belief hierarchies that continue to reason beyond order $\ell$ and that stop reasoning at that order, respectively. Define

$$
\begin{aligned}
& \widetilde{H}_{i}^{\leq k}:=\widetilde{H}_{i}^{k} \cup \bigcup_{\ell=0}^{k} H_{i}^{\ell}, \quad \widetilde{\Omega}_{i}^{k}:=\Theta \times \widetilde{H}_{-i}^{\leq k}, \\
& H_{i}^{\leq k}:=\bigcup_{\ell=0}^{k} H_{i}^{\ell}, \quad \Omega_{i}^{k}:=\Theta \times H_{-i}^{\leq k},
\end{aligned}
$$

and let

$$
\begin{aligned}
\widetilde{H}_{i}^{k+1} & :=\left\{\left(x_{i}, \mu_{i}^{0}, \ldots, \mu_{i}^{k}, \mu_{i}^{k+1}\right) \in \widetilde{H}_{i}^{k} \times \Delta\left(\widetilde{\Omega}_{i}^{k}\right): \operatorname{marg}_{\widetilde{\Omega}_{i}^{k-1}} \mu_{i}^{k+1}=\mu_{i}^{k}\right\}, \\
H_{i}^{k+1} & :=\left\{\left(x_{i}, \mu_{i}^{0}, \ldots, \mu_{i}^{k}, \mu_{i}^{k+1}\right) \in \widetilde{H}_{i}^{k} \times \Delta\left(\Omega_{i}^{k}\right): \operatorname{marg}_{\widetilde{\Omega}_{i}^{k-1}} \mu_{i}^{k+1}=\mu_{i}^{k}\right\} .
\end{aligned}
$$

Again, the interpretation is that $\widetilde{H}_{i}^{k+1}$ is the set of belief hierarchies that continue to reason at order $k+1$, while the set $H_{i}^{k+1}$ contains the hierarchies that stop reasoning at $k+1$. As before, the former can conceive of the possibility that the other players have not stopped reasoning at order $k$, while the latter cannot. A belief hierarchy $h_{i}^{k} \in H_{i}^{k}$ that stops reasoning at order $k$ is said to have depth (of reasoning) $k$. The condition on the marginal in (1) and (2) is a standard coherency condition: it ensures that the beliefs at different orders do not contradict each other (see, e.g., Dekel and Siniscalchi 2015, for a discussion). Define

$$
H_{i}^{\infty}:=\left\{\left(x_{i}, \mu_{i}^{0}, \mu_{i}^{1}, \ldots\right):\left(x_{i}, \mu_{i}^{0}, \ldots, \mu_{i}^{k}\right) \in \widetilde{H}_{i}^{k} \text { for all } k \geq 0\right\} .
$$

The belief hierarchies in $H_{i}^{\infty}$ are those that "reason up to infinity." We therefore say that a belief hierarchy $h_{i}^{\infty}$ in $H_{i}^{\infty}$ has an infinite depth (of reasoning). The set of all belief hierarchies is thus ${ }^{15}$

$$
H_{i}:=H_{i}^{\infty} \cup \bigcup_{k=0}^{\infty} H_{i}^{k} .
$$

\footnotetext{
${ }^{15}$ As noted in Section 3.1, the union $H_{i}$ is endowed with the sum topology. In particular, the set $H_{i}^{\infty}$ of types with an infinite depth is open. This ensures that a sequence of types with finite but increasing depth does not converge to a standard type. Our results do not depend on this in any way.
} 
With some abuse of notation, we sometimes write $\left(x_{i}, \mu_{i}^{0}, \ldots\right)$ for an element $h_{i}$ of $H_{i}$, regardless of whether $h_{i}$ has finite or infinite depth.

\subsection{Universal type space}

Following Mertens and Zamir (1985), we can use the sets $H_{i}$ of all belief hierarchies to define the universal type space. A key observation is that every belief hierarchy $h_{i}$ corresponds to a belief $\psi_{i}\left(h_{i}\right) \in \Delta\left(\Theta \times H_{-i}\right)$ about nature and other players' hierarchies.

Proposition 1. There is unique mapping $\psi_{i}: H_{i} \rightarrow\left\{h_{i}^{*, 0}\right\} \cup \Delta\left(\Theta \times H_{-i}\right)$ with the property that for each $k=1,2, \ldots$, for each $h_{i}=\left(x_{i}, \mu_{i}^{1}, \ldots\right) \in H_{i}$ of depth at least $k$, its $k$ thorder belief $\mu_{i}^{k}$ is given by

$$
\mu_{i}^{k}=\operatorname{marg}_{\widetilde{\Omega}_{i}^{k-1}} \psi_{i}\left(h_{i}\right)
$$

and is such that

- $f o r h_{i}^{0} \in H_{i}^{0}, \psi_{i}\left(h_{i}^{0}\right)=h_{i}^{*, 0}$,

- for $h_{i}^{k} \in H_{i}^{k}, k<\infty$, the support of $\psi_{i}\left(h_{i}^{k}\right)$ lies in $\Theta \times H_{-i}^{\leq k-1}$,

- for $h_{i}^{\infty} \in H_{i}^{\infty}$, the support of $\psi_{i}\left(h_{i}^{\infty}\right)$ lies in $\Theta \times H_{-i}$.

Moreover, the function $\psi_{i}$ is continuous.

The result follows from Proposition 9 in Appendix B. There we show that the tuple $\left(H_{i}, \psi_{i}\right)_{i=1,2}$ defines a type space, denoted $\mathcal{T}^{*}$, with $H_{i}$ a set of types and for each type $h_{i} \in H_{i}$, a belief $\psi_{i}\left(h_{i}\right) \in \Delta\left(\Theta \times H_{-i}\right)$ over the payoff parameters and the other player's types. As we show, $\mathcal{T}^{*}$ is universal in the sense that it generates all belief hierarchies with a finite or infinite depth. For simplicity, we sometimes write $\psi_{h_{i}}$ for the belief $\psi\left(h_{i}\right)$ associated with the type $h_{i}$.

In some instances, a researcher may want to rule out certain beliefs. For example, in an auction setting, he may want to assume that a player's expected valuation increases in his signal. This can be captured using models.

Definition 1. A model is a pair $M=(\widetilde{\Theta}, T)$, where $\widetilde{\Theta} \subset \Theta$ and $T:=\left(T_{i}\right)_{i=1,2}$ is a pair of subsets $T_{i} \subset H_{i}$ of types such that for each type $h_{i} \in T_{i} \backslash H_{i}^{0}, \psi_{h_{i}}$ has support in $\widetilde{\Theta} \times T_{-i}$. A model is finite if $T$ is finite.

Note that whether a model is finite is unrelated to the depth of reasoning of the types. For example, a finite model may include only infinite-depth types, and a model that is not finite may consist of types of any (finite or infinite) depth.

\section{5 (Almost-) common belief in an infinite depth}

We are interested in relaxing the standard assumptions on beliefs about players' depth of reasoning. We first make these assumptions explicit in the context of the universal 
type space $\mathcal{T}^{*}$. Define

$$
C_{i}^{1}:=\left\{h_{i} \in H_{i}^{\infty}: \psi_{h_{i}}\left(\Theta \times H_{-i}^{\infty}\right)=1\right\}
$$

to be the set of types that have an infinite depth of reasoning and that believe that the opponent has an infinite depth. Since types from standard (Harsanyi) type spaces all generate belief hierarchies with an infinite depth, standard types all satisfy this assumption. For $n>1$, let

$$
C_{i}^{n}:=\left\{h_{i} \in C_{i}^{n-1}: \psi_{h_{i}}\left(\Theta \times C_{-i}^{n-1}\right)=1\right\}
$$

be the set of infinite-depth types that have $n$ th-order mutual belief in an infinite depth. Again, all types from standard type spaces satisfy this condition. Let $C_{i}^{\infty}:=\bigcap_{n} C_{i}^{n}$. Then $C^{\infty}:=\left(C_{i}^{\infty}\right)_{i=1,2}$ is the event in $\mathcal{T}^{*}$ that types have an infinite depth of reasoning and there is common (correct) belief in the event that players have an infinite depth of reasoning. It is easy to see that $C^{\infty}$ is a model. The next result states that the types from standard type spaces are precisely the types in $C^{\infty}$.

Proposition 2 (Common belief in infinite depth). The universal type space $\mathcal{T}^{\mathrm{MZ}}$ of Mertens and Zamir (1985) corresponds to the event in $\mathcal{T}^{*}$ that players have an infinite depth of reasoning and this is common belief: there is a belief-preserving homeomorphism from $\mathcal{T}^{\mathrm{MZ}}$ to $\mathrm{C}^{\infty}$.

See the Supplemental Appendices (available in a supplementary file on the journal website, http://econtheory.org/supp/2098/supplement.pdf) for a formal statement and the proof. With this characterization in hand, we can weaken the strong assumption that there is common belief in an infinite depth by allowing for small deviations from this assumption. We thus consider the event that players have an infinite depth of reasoning and this is almost-common belief. To construct this event, we define a sequence $B_{i}^{1}, B_{i}^{2}, \ldots$ of subsets of types. For each player $i$, let

$$
B_{i}^{0}:=\left\{h_{i} \in H_{i}^{\infty}: \psi_{h_{i}}\left(\bigcup_{\gamma<\infty} H_{-i}^{\gamma}\right)=1\right\}
$$

be the set of types that have an infinite depth and that believe (with probability 1) that the other player has a finite depth. For $n=1,2, \ldots$, define

$$
B_{i}^{n}:=\left\{h_{i} \in H_{i}^{\infty}: \psi_{h_{i}}\left(B_{-i}^{n-1}\right)=1\right\} .
$$

Thus, if player $i$ has a type in $B_{i}^{1}$, then he has an infinite depth of reasoning, and he believes that his opponent has an infinite depth but that she believes that he has a finite depth. Generally, the types in $B_{i}^{n}$ have an infinite depth of reasoning and have $n$ thorder mutual belief in the event that players have an infinite depth of reasoning, but not $(n+1)$ th-order mutual belief in that event.

We also consider the analogous conditions under the assumption that players have level- $k$ beliefs. Level- $k$ beliefs have been explored in the experimental literature. The assumption is that players with finite depth $\ell$ believe that their opponents have depth $\ell-1$. 
For $\ell=1,2, \ldots$, let $G_{i}^{\ell} \subset H_{i}^{\ell}$ be the set of types for player $i$ that believe that his opponent has depth $\ell-1$, believe that his opponent believes that he has depth $\ell-1-1$, and so on (i.e., $\psi_{h_{i}}\left(G_{-i}^{\ell-1}\right)=1$ for $\left.h_{i} \in G_{i}^{\ell}\right)$. Define

$$
\widetilde{B}_{i}^{0}:=\left\{h_{i} \in H_{i}^{\infty}: \psi_{h_{i}}\left(\bigcup_{\gamma<\infty} G_{-i}^{\gamma}\right)=1\right\},
$$

and for $n=1,2, \ldots$, define $\widetilde{B}_{i}^{n}$ analogously to $B_{i}^{n}$. Clearly, $\widetilde{B}^{n} \subset B^{n}$. Like $B^{0}, B^{1}, \ldots$, the sequence $\widetilde{B}^{0}, \widetilde{B}^{1}, \ldots$ captures that players have an infinite depth of reasoning and have almost-common belief in the event that players have an infinite depth of reasoning; in addition, the only finite-depth types that types in $\widetilde{B}^{n}$ think possible have level- $k$ beliefs.

\subsection{Rationalizability}

We next extend the standard definition of rationalizability to our environment. As we show in Appendix A, it suffices to define rationalizability for the universal type space $\mathcal{T}^{*}$ since the set of rationalizable actions of a type depends only on its induced belief hierarchy, as in the standard case (Dekel et al. 2007). For each player $i=1,2$ and $h_{i} \in$ $H_{i} \backslash H_{i}^{0}$, define

$$
R_{i}^{0}\left(h_{i}\right):=A_{i}
$$

For $m>1$, define inductively ${ }^{16}$

$$
R_{i}^{m}\left(h_{i}\right):=\left\{\begin{array}{c}
\text { there is a measurable } s_{-i}: \Theta \times H_{-i} \rightarrow \Delta\left(A_{-i}\right) \text { s.t. } \\
a_{i} \in A_{i}: \operatorname{supp} s_{-i}\left(\theta, h_{-i}\right) \subseteq R_{-i}^{m-1}\left(h_{-i}\right) \text { for all } h_{-i} \in H_{-i}, \theta \in \Theta ; \text { and } \\
a_{i} \in \arg \max _{a_{i}^{\prime} \in A_{i}} \int_{\Theta \times H_{-i} \times A_{-i}} u_{i}\left(a_{i}^{\prime}, a_{-i}, \theta\right) s_{-i}\left(\theta, h_{-i}\right)\left(a_{-i}\right) \mathrm{d} \psi_{h_{i}}
\end{array}\right\},
$$

where supp $\mu$ is the support of a probability measure $\mu$. Then $R_{i}^{m}\left(h_{i}\right)$ is the set of $m$ rationalizable actions for $h_{i}$. The $m$-rationalizable actions for a type are the actions that survive $m$ rounds of iterated deletion of dominated actions: for each action $a_{i} \in R_{i}^{m}\left(h_{i}\right)$, there is a conjecture $s_{-i}$ that rationalizes it, in the sense that the conjecture has support in the actions of the opponents that have survived $m-1$ rounds of deletion, and $a_{i}$ is a best response to this conjecture (given the type's belief $\psi_{h_{i}}$ ). The (interim correlated) rationalizable actions of type $h_{i}$ are

$$
R_{i}^{\infty}\left(h_{i}\right):=\bigcap_{m=0}^{\infty} R_{i}^{m}\left(h_{i}\right)
$$

If $h_{i} \in H_{i}^{0}$, then we set $R_{i}^{\infty}\left(h_{i}\right):=A_{i}$.

For types that have an infinite depth of reasoning and common belief in this event, interim correlated rationalizability captures the behavioral implications of rationality and common belief in rationality (Dekel et al. 2007). If a type has finite depth $k$, then its set of rationalizable actions is completely determined by the actions that survive $k$ rounds of elimination.

\footnotetext{
${ }^{16}$ For notational convenience, the conjecture $s_{-i}$ in the definition of $R_{i}^{m}\left(h_{i}\right)$ is defined also for types $h_{-i}$ outside the support of $\psi_{h_{i}}$. This does not affect our results.
} 
Lemma 1. Let $h_{i}$ be a type with finite depth $k$. Then an action $a_{i}$ is $k$-rationalizable for $h_{i}$ if and only if it is rationalizable for the type.

In Appendix C, we show that rationalizability satisfies the standard best-reply property: any rationalizable action for a type is a best response to the belief that the opponent chooses a rationalizable strategy (cf. Dekel et al. 2007, Proposition 4).

The rationalizability correspondence satisfies a standard upper hemicontinuity property (cf. Dekel et al. 2007, Lemma 1).

Lemma 2 (Upper hemicontinuity). The rationalizability correspondence is nonempty and upper hemicontinuous: every type $h_{i} \in H_{i}$ has a neighborhood $O\left(h_{i}\right)$ such that $R_{i}^{\infty}\left(h_{i}\right) \supset R_{i}^{\infty}\left(h_{i}^{\prime}\right) \neq \varnothing$ for $h_{i}^{\prime} \in O_{i}\left(h_{i}\right)$. Likewise, the $m$-rationalizability correspondence is nonempty and upper hemicontinuous.

The result says that if a player's actual type is $h_{i}$ but a researcher has only an imperfect observation of the player's type (i.e., he observes that the type is in $O_{i}\left(h_{i}\right)$ ), then any action that is rationalizable for a type that is consistent with his observation (i.e., $a_{i} \in R_{i}^{\infty}\left(h_{i}^{\prime}\right)$ for $\left.h_{i}^{\prime} \in O_{i}\left(h_{i}\right)\right)$ is rationalizable for the player's actual type (i.e., $a_{i} \in R_{i}^{\infty}\left(h_{i}\right)$ ), and likewise for finite-order rationalizability.

A researcher who cannot perfectly observe the players' types may want his predictions to be robust to small perturbations in beliefs. The concept of robust rationalizability captures such a robustness requirement.

Definition 2. A subset $A_{i}^{\prime} \subset A_{i}$ of actions is robustly rationalizable for a type $h_{i}$ if the rationalizable actions for $h_{i}$ are precisely the actions in $A_{i}^{\prime}$ and $R_{i}^{\infty}$ is locally constant at $h_{i}$ : $h_{i}$ has a neighborhood $O_{i}\left(h_{i}\right)$ such that $R_{i}^{\infty}\left(h_{i}^{\prime}\right)=A_{i}^{\prime}$ for every $h_{i}^{\prime} \in O_{i}\left(h_{i}\right)$. Likewise, $A_{i}^{\prime}$ is robustly $m$-rationalizable for $h_{i}$ if the $m$-rationalizable actions for $h_{i}$ are precisely the actions in $A_{i}^{\prime}$ and $R_{i}^{m}$ is locally constant at $h_{i}$.

A direct corollary of Lemma 2 is that uniqueness is robust.

COROLLARY 1 (Uniqueness robust). If action $a_{i}$ is the unique rationalizable action for a type $h_{i}$, then $\left\{a_{i}\right\}$ is robustly rationalizable for $h_{i}$.

Weinstein and Yildiz (2007) show the surprising result that if players have an infinite depth and this is common belief, then only uniqueness is robust.

Proposition 3 (No robust multiplicity under common belief in an infinite depth; Weinstein and Yildiz 2007, Proposition 2). Under Assumption R-Dom, $A_{i}^{\prime}$ is robustly rationalizable for a type $h_{i}$ with common belief in an infinite depth (i.e., $h_{i} \in C_{i}^{\infty}$ ) only if $A_{i}^{\prime}$ is a singleton.

Thus, if a researcher thinks that players have an infinite depth of reasoning and this is common belief, then the only robust predictions he can make is that players have a unique rationalizable action. In the next section, we show that this extreme conclusion is not robust to relaxing the assumption that there is common belief in an infinite depth of reasoning. 


\section{Robust multiplicity}

In this section, we show that the rationalizable actions of a type $h_{i}$ with multiple rationalizable actions can be robust to perturbations of beliefs at $h_{i}$ when there is uncertainty about players' depth of reasoning. Section 4.1 considers the general case to derive basic insights on the rationalizability correspondence. Section 4.2 focuses on the case of complete-information types to show that, unlike in the standard case with common belief in an infinite depth, the rationalizability correspondence is continuous around complete-information types when we relax standard assumptions. Section 5 builds on the insights developed in this section to develop robust refinements.

\subsection{General}

We first consider general types. The main result of this section shows that if we relax the assumption that it is common belief that players have an infinite depth of reasoning, then multiplicity can be robust.

Proposition 4 (Robust multiplicity under almost-common belief in infinite depth). Under Assumption R-Mult $\left(A^{\prime}\right)$, for every $m=1,2, \ldots$, there is an infinite-depth type $h_{i}^{m}$ with mth-order mutual belief in an infinite depth for whom $A_{i}^{\prime}$ is robustly rationalizable.

Proof. For simplicity, we say that a type $h_{i}$ has a belief in the multiplicity set if $\operatorname{marg}_{\Theta} \psi_{h_{i}} \in \Delta_{i}^{A^{\prime}}$ (where $\Delta_{i}^{A^{\prime}}$ is defined under Assumption R-Mult $\left(A^{\prime}\right)$ ), and we write $h_{i} \in \Delta_{i}^{A^{\prime}}$. The proof has two key ingredients. The first is used to construct a "grain" of robust multiplicity.

Lemma 3. Under Assumption R-Mult( $\left(A^{\prime}\right)$, the set $\left\{h_{i} \in H_{i}: h_{i} \in \Delta_{i}^{A^{\prime}}\right\}$ is nonempty and open.

The set in Lemma 3 can be used to construct a grain of robust multiplicity in the sense that it almost immediately defines a set of depth-1 types with robust multiplicity, as we show below. The second key ingredient provides us with a contagion-type argument: given a grain $V$ of robust multiplicity, we can identify other types with robust multiplicity.

Lemma 4 (Contagion). Under Assumption $R$-Mult $\left(A^{\prime}\right)$, for $m=0,1,2, \ldots, \infty$, if $V \subset H_{-i}$ is an open subset of types such that $R_{-i}^{m}\left(h_{-i}\right)=A_{-i}^{\prime}$ for $h_{-i} \in V$, then every type $h_{i}$ with a belief in the multiplicity set that assigns probability 1 to $V$ has a neighborhood $O\left(h_{i}\right)$ such that $R_{i}^{m+1}\left(h_{i}^{\prime}\right)=A_{i}^{\prime}$ for all $h_{i}^{\prime} \in O\left(h_{i}\right)($ where $\infty+1=\infty)$.

Lemma 4 says that if there is an open set $V$ of types for whom the set of rationalizable actions is $A^{\prime}$, then $A^{\prime}$ is robustly rationalizable for any type that has a belief in the multiplicity set and that believes that the opponent has a type in $V$, and similarly for finite-order rationalizability.

With these tools in hand, we can prove Proposition 4 . The first step is to show that $A^{\prime}$ is robustly $m$-rationalizable for any finite $m$. 
Lemma 5. Under Assumption R-Mult $\left(A^{\prime}\right)$, for every $m=1,2, \ldots$, there are types for whom the actions in $A_{i}^{\prime}$ are robustly $m$-rationalizable.

The proof of Lemma 5 can be found in Appendix D. The proof uses Lemma 3 to show that the set of types for whom $A^{\prime}$ is 1-rationalizable is open. This gives a set of types for whom $A^{\prime}$ is robustly 1-rationalizable. The proof then applies Lemma 4 repeatedly to identify sets of types for whom $A^{\prime}$ is robustly $m$-rationalizable.

Together with Lemma 1, Lemma 5 immediately implies that multiplicity is robust for finite-depth types.

Corollary 2 (Grain of robust multiplicity). Under Assumption R-Mult $\left(A^{\prime}\right)$, for every $m=1,2, \ldots$, there are depth- $m$ types for whom $A_{i}^{\prime}$ is robustly rationalizable. In fact, for every $m=1,2, \ldots$, there is an open set $V_{i}^{m} \subset H_{i}^{m}$ of depth-m types such that $R_{i}^{\infty}\left(h_{i}\right)=A_{i}^{\prime}$ for $h_{i} \in V_{i}^{m}$.

The second claim in Corollary 2 follows immediately from the first: by the first claim, there is an open set $O_{i}^{m}$ of types for whom $A_{i}^{\prime}$ is robustly rationalizable that satisfies $O_{i}^{m} \cap$ $H_{i}^{m} \neq \varnothing$. The second claim then follows by taking $V_{i}^{m}:=O_{i}^{m} \cap H_{i}^{m}$. It is straightforward to use the contagion argument in Lemma 4 to show that multiplicity can be robust for types with an infinite depth that have almost-common belief in an infinite depth; see the Appendix.

The contrast between the negative result for the case with common belief in an infinite depth (Proposition 3) and the positive result for the case with almost-common belief in an infinite depth (Proposition 4) is stark: if the set of possible payoff functions is sufficiently rich (i.e., satisfies Assumptions R-Dom and R-Mult $\left(A^{\prime}\right)$ ), then multiplicity is not robust when it is common belief that players have an infinite depth of reasoning, yet it can be robust if there is almost-common belief in an infinite depth of reasoning. The deviation from standard assumptions is arguably minimal: the types in Proposition 4 are arbitrarily close to standard types. We could even make more stringent assumptions on players' beliefs about reasoning ability. For example, the result holds also if we require that players have level- $k$ beliefs (i.e., if we replace $B^{n}$ by $\widetilde{B}^{n}$ throughout) or require that infinite-depth types assign high probability only to types with a high depth of reasoning. In fact, the same result obtains in a universal type space in which every type has depth at least $k$ for arbitrary finite $k$.

As noted in Section 2, the key difference between the current framework and the standard case is that the universal type space now contains a grain of robust multiplicity consisting of finite-depth types (Corollary 2). ${ }^{17}$ Robust multiplicity for these finitedepth types follows almost immediately from robust multiplicity under finite-order rationalizability (Lemma 5) and the fact that the rationalizable actions for a type of depth $m$ do not depend on its beliefs beyond order $m$ (Lemma 1).

\footnotetext{
${ }^{17}$ The contagion argument, Lemma 4, goes through also if there is common belief in an infinite depth. Hence, the analogue of Lemma 5 for $\mathcal{T}^{\mathrm{MZ}}$ also holds: under Assumption R-Mult $\left(A^{\prime}\right)$, for every $m=1,2, \ldots$, there are types in the universal space $\mathcal{T}^{\mathrm{MZ}}$ for standard types for whom the actions in $A^{\prime}$ are robustly $m$-rationalizable.
} 
This robust multiplicity extends beyond types with a finite depth to types with an infinite depth and almost-common belief in an infinite depth. Intuitively, even for a type with an infinite depth and $n$ th-order mutual belief in infinite depth (for arbitrarily high but finite $n$ ), belief perturbations at high orders have only a small effect. To see this, consider a nonsingleton set $A^{\prime}$ of action profiles and type $h_{i}^{\mathrm{MZ}} \in C_{i}^{\infty}$ with common belief in an infinite depth whose belief lies in the multiplicity set $\Delta_{i}^{A^{\prime}}$ and that satisfies common belief in the event that players' beliefs are in the multiplicity set for $A^{\prime}$. Since the action set is finite, there is $m<\infty$ such that $R_{i}^{\infty}\left(h_{i}^{\mathrm{MZ}}\right)=R_{i}^{m}\left(h_{i}^{\mathrm{MZ}}\right)=A_{i}^{\prime}$. By the results of Weinstein and Yildiz (2007), multiplicity is not robust for $h_{i}^{\mathrm{MZ}}$ (Proposition 3). Now consider a type $h_{i} \notin C_{i}^{\infty}$ that has the same $m$ th-order belief hierarchy as $h_{i}$ but believes that the other player has a finite depth. Then, since $h_{i}$ has the same $m$ th-order belief hierarchy as $h_{i}^{\mathrm{MZ}}, R_{i}^{\infty}\left(h_{i}\right)=R_{i}^{\infty}\left(h_{i}^{\mathrm{MZ}}\right)=A_{i}^{\prime}$ (Lemma 7). If $h_{i}$ assigns probability 1 to types with depth less than $k<\infty$, then it is immediate that the researcher's prediction is robust whenever he can observe the type's beliefs up to order $k+1$. So suppose that type $h_{i}$ has an infinite depth and assigns positive probability to types with an arbitrarily high (but finite) depth of reasoning. Then, for increasing $k, h_{i}$ must put vanishingly small weight on types with depth greater than $k$. Hence, by observing sufficiently many orders of beliefs, the researcher can thus rule out that his prediction depends sensitively on the type's (unobserved) high order beliefs and he can be confident in his prediction that the rationalizable actions for the type are precisely the actions in $A_{i}^{\prime}$ even if he does not observe the type's entire hierarchy of beliefs. This argument extends to types that believe that the opponent believes that the other player has a finite depth, to types that believe that the opponent believes that the other player believes that their opponent has a finite depth, etc.

This reveals a subtle way in which a small "grain of naiveté" can give rise to robust predictions: multiplicity can be robust for a type close to standard types if its belief hierarchy is finitely determined in the sense that either the type has a finite depth, or thinks it is likely that the other player does not continue to reason beyond a certain order, or thinks that it is likely that the other player thinks this is likely, and so on. Thus, by making more precise observations, a researcher can make robust predictions if he learns that the type has a finite depth, or that it assigns low probability to the other player continuing to reason at high orders, or that it assigns low probability to the other player assigning high probability to her opponent continuing to reason at high orders, and so on.

A direct implication of Proposition 4 is that, unlike in the standard case where types generically have a unique rationalizable action (Weinstein and Yildiz 2007), uniqueness is not generic if types can have an arbitrary depth of reasoning. ${ }^{18}$

In Section 5, we build on these insights to show that robust refinements of rationalizability exist. Before exploring this, we apply the insights developed in this section to show that there need not be a discontinuity of behavior around complete-information types when we relax the assumption that there is common belief in an infinite depth.

\footnotetext{
${ }^{18}$ Formally, Proposition 4 shows that the set of types with multiple rationalizable actions has a nonempty interior in the universal type space $\mathcal{T}^{*}$. This implies that the set of types with a unique rationalizable actions is not generic (i.e., is not open and dense). However, Proposition 4 is strictly stronger. For example, the set $\mathbb{Q}$ of rationals is not open and dense in the set $\mathbb{R}$ of real numbers, yet the interior of its complement $\mathbb{R} \backslash \mathbb{Q}$ is empty.
} 


\subsection{Almost-complete information}

The same basic tools can be used to study robustness in specific applications. We focus on the case where payoffs are observed with some small noise. Again, consider the well known example from Carlsson and van Damme (1993),

\begin{tabular}{c|c|c|}
\multicolumn{1}{c}{} & \multicolumn{1}{c}{$I$} & \multicolumn{1}{c}{$N I$} \\
\cline { 2 - 3 }$I$ & $\theta, \theta$ & $\theta-1,0$ \\
\cline { 2 - 3 }$N I$ & $0, \theta-1$ & 0,0 \\
\cline { 2 - 3 } & &
\end{tabular}

where $\theta \in \Theta:=[-1,2]$. Both Assumptions R-Dom and R-Mult $\left(A^{\prime}\right)$ (with $A_{i}^{\prime}=\{I, N I\}$ ) are satisfied: If $\theta>1$, then investing $(I)$ is strictly dominant for each player; if $\theta<0$, then not investing $(N I)$ is strictly dominant. For $\theta \in(0,1)$, either action is a strict best response for a player, depending on his conjecture about the opponent's play.

The information structure is as described in Example 1. That is, each player $i$ observes signal $x_{i}$ of the state $\theta$ that is potentially noisy. The noise is measured by a parameter $\varepsilon \in\left[0, \frac{1}{2}\right)$. Players' observations of $\theta$ become increasingly precise as the noise parameter $\varepsilon$ goes to 0 . It will be convenient to take the set of signals to be $X_{i}:=[-2,3]$. When the noise parameter is $\varepsilon$, the set of signals that types receive with positive probability is thus $X_{i}^{\varepsilon}:=[-1-\varepsilon, 2+\varepsilon]$.

We consider a simple model with level- $k$ beliefs that is consistent with this information structure. For each signal $x_{i} \in X_{i}^{\varepsilon}$, there is a unique type $h_{i}^{1, x_{i}}$ in $H_{i}^{1}$ with signal $x_{i}$ whose beliefs are consistent with the information structure. Let $T_{i}^{\varepsilon, 1}:=\left\{h_{i}^{0, x_{i}}: x_{i} \in X_{i}^{\varepsilon}\right\}$ be the set of such types. For $m>1$, suppose $T_{i}^{\varepsilon, m-1}$ is a set of depth-(m-1) types with beliefs consistent with the information structure such that for every $x_{i} \in X_{i}^{\varepsilon}$, there is a type in $T_{i}^{\varepsilon, m-1}$ with signal $x_{i}$. Then, for each signal $x_{i} \in X_{i}^{\varepsilon}$, there is a unique depth$m$ type $h_{i}^{m, x_{i}}$ with signal $x_{i}$ whose beliefs are consistent with the information structure and that assigns probability 1 to $T_{-i}^{\varepsilon, m-1}$. Let $T_{i}^{\varepsilon, m}:=\left\{h_{i}^{m, x_{i}}: x_{i} \in X_{i}^{\varepsilon}\right\}$ be the set of such types. We next define the types with an infinite depth of reasoning. Fix some vector $\left(p_{1}, p_{2}, \ldots\right)$ of probabilities (i.e., $p_{n} \geq 0$ for all $n$, and $\sum_{n} p_{n}=1$ ). Then, for each $x_{i} \in X_{i}^{\varepsilon}$, there is a unique infinite-depth type $h_{i}^{\infty, x_{i}}$ in $\widetilde{B}_{i}^{0}$ with signal $x_{i}$ whose beliefs are consistent with the information structure that assigns probability $p_{n}$ to $T_{-i}^{\varepsilon, n}, n=1,2, \ldots$ Let $T_{i}^{\varepsilon, \infty}:=\left\{h_{i}^{\infty, x_{i}}: x_{i} \in X_{i}^{\varepsilon}\right\}$. For $n>0$, given a set $T_{i}^{\varepsilon, \infty+n-1} \subset \widetilde{B}_{i}^{n-1}$ of types with beliefs consistent with the information structure, we can likewise select, for each $x_{i} \in X_{i}^{\varepsilon}$, the unique type $h_{i}^{\infty+n, x_{i}}$ in $\widetilde{B}_{i}^{n}$ with signal $x_{i}$ whose beliefs are consistent with the information structure; this defines a set $T_{i}^{\varepsilon, \infty+n}$ of types with an infinite depth and $n$ thorder mutual belief in an infinite depth whose beliefs are consistent with the information structure. Let $T_{i}^{\varepsilon}$ be the union of the sets $T_{i}^{\varepsilon, 0}, T_{i}^{\varepsilon, 1}, \ldots, T_{i}^{\varepsilon, \infty}, T_{i}^{\varepsilon, \infty+1}, \ldots$ (where $\left.T_{i}^{\varepsilon, 0}:=X_{i}^{\varepsilon} \times\left\{h_{i}^{*, 0}\right\}\right)$. Then $M^{\varepsilon}=\left(\Theta, T^{\varepsilon}\right)$ is a model with level- $k$ beliefs that is consistent with the information structure. Moreover, it is consistent with almost-common belief in an infinite depth (i.e., for all $n, \widetilde{B}^{n} \cap T^{\varepsilon} \neq \varnothing$ ).

A first observation is that for any signal $x_{i} \in(0,1)$, both actions are rationalizable for finite-depth types in $M^{\varepsilon}$ with signal $x_{i}$, provided that the noise is sufficiently small. 
Leмma 6. For every $k$, there exist $\underline{x}_{k}^{\varepsilon} \in\left(0, \frac{1}{2}\right)$ and $\bar{x}_{k}^{\varepsilon} \in\left(\frac{1}{2}, 1\right)$ such that for every depth- $k$ type $h_{i}$ in $T^{\varepsilon}$, both actions are robustly rationalizable whenever its signal $x_{i}$ is in $\left(\underline{x}_{k}^{\varepsilon}, \bar{x}_{k}^{\varepsilon}\right)$. Moreover, for every $k, \underline{x}_{k}^{\varepsilon} \rightarrow 0$ and $\bar{x}_{k}^{\varepsilon} \rightarrow 1$ as $\varepsilon \rightarrow 0$.

The proof gives an explicit expression for the interval bounds $\underline{x}_{k}^{\varepsilon}$ and $\bar{x}_{k}^{\varepsilon}$. The next result extends this to the case where types have an infinite depth and have almostcommon belief in infinite depth.

Proposition 5 (Robust multiplicity around complete-information types). For every $m$, there exist $\varepsilon_{m}>0, \underline{x}_{m}^{\varepsilon} \in\left(0, \frac{1}{2}\right)$, and $\bar{x}_{m}^{\varepsilon} \in\left(\frac{1}{2}, 1\right)$ such that both actions are robustly rationalizable for any infinite-depth type $h_{i} \in T^{\varepsilon}$ with signal $x_{i} \in\left(\underline{x}_{m}^{\varepsilon}, \bar{x}_{m}^{\varepsilon}\right)$ and mth-order belief in an infinite depth whenever $\varepsilon<\varepsilon_{m}$. Moreover, we can choose the bounds $\underline{x}_{m}^{\varepsilon}$ and $\bar{x}^{\varepsilon}$ such that $\underline{x}_{m}^{\varepsilon} \rightarrow 0$ and $\bar{x}_{m}^{\varepsilon} \rightarrow 1$ as $\varepsilon \rightarrow 0$.

Proposition 5 implies that the strategic discontinuity around complete-information games is not robust to relaxing the assumption that there is common belief in an infinite depth of reasoning. Proposition 5 shows that there are models in which there is a small amount of uncertainty about players' depth of reasoning such that players can rationally choose both actions when neither action is dominant when noise $\varepsilon$ goes to 0 , just as in the limit case with complete information (i.e., $\varepsilon=0$ ). In other words, there is no discontinuity when $\varepsilon$ goes to 0 in these models. In particular, the risk-dominant strategy is not uniquely selected when $\varepsilon$ is small but positive, unlike in the standard case (Carlsson and van Damme 1993).

Importantly, the conclusions in Proposition 5 are robust to further belief perturbations. Since multiplicity is robust, the predictions remain valid even if the researcher has misspecified the model in the sense that the model $T^{\varepsilon}$ does not accurately describe players' beliefs, as long as the assumptions embodied in the model are satisfied approximately.

The proof of Proposition 5 is similar in nature to that of Proposition 4: the key ingredients are a "grain" of robust multiplicity, provided by the finite-depth types, and a contagion argument to establish robust multiplicity for types consistent with almostcommon belief in infinite depth. The proofs differ slightly in that the contagion argument for Proposition 5 has to ensure that beliefs are consistent with the information structure. The proof of Proposition 5 thus illustrates how the basic contagion argument can be adapted to prove results for specific applications.

Again, Proposition 5 does not rely on strong assumptions on players' beliefs. First, it does not require that players believe that other players have a shallow depth of reasoning, or that others believe that, believe that others believe that, and so on. Indeed, the same result obtains if we assume that all players have depth at least $k$ for arbitrary finite $k$. Second, while $T^{\varepsilon}$ is a minimal model consistent with the information structure and almost-common belief in an infinite depth (assuming level- $k$ beliefs), the main insight extends to a much broader range of situations. It can be shown that multiplicity can be robust for all types whose beliefs are consistent with the information structure and almost-common belief in an infinite depth, though the bound on the noise level may depend on the detailed features of a type in that case. 


\section{Robust REFinements}

In this section, we study the implications of robust multiplicity for the scope of robust refinements. We show that, unlike in the standard case with common belief in an infinite depth, robust refinements do exist when there is almost-common belief in an infinite depth.

\subsection{Definitions}

We start by defining some basic concepts. A strategy for a model $M=(\widetilde{\Theta}, T)$ is a measurable function $\sigma_{i}$ that maps each type $h_{i} \in T_{i}$ into a mixed action $\sigma_{i}\left(h_{i}\right) \in \Delta\left(A_{i}\right)$. We write $\sigma_{i}\left(a_{i} \mid h_{i}\right)$ for the probability that $i$ plays $a_{i}$ when his type is $h_{i}$. A strategy profile $\sigma=\left(\sigma_{i}\right)_{i=1,2}$, with $\sigma_{i}$ a strategy for $M$, is a (Bayesian-Nash) equilibrium for $M$ if, for each player $i=1,2, h_{i} \in T_{i} \backslash H_{i}^{0}, a_{i} \in A_{i}$ such that $\sigma_{i}\left(a_{i} \mid h_{i}\right)>0$,

$$
\int u_{i}\left(a_{i}, \sigma_{-i}\left(h_{-i}\right), \theta\right) \mathrm{d} \psi_{h_{i}} \geq \int u_{i}\left(a_{i}^{\prime}, \sigma_{-i}\left(h_{-i}\right), \theta\right) \mathrm{d} \psi_{h_{i}}
$$

for all $a_{i}^{\prime} \in A_{i}$. As before, a nonstrategic type $h_{i} \in H_{i}^{0}$ can play any action. An equilibrium is strict if the above inequality is strict whenever $a_{i}^{\prime} \neq a_{i}$. Bayesian-Nash equilibrium refines rationalizability: if $\sigma$ is a Bayesian-Nash equilibrium for $M$, then every action $a_{i}$ that is played with positive probability by a type $h_{i}$ in $M$ under $\sigma$ (i.e., $\sigma_{i}\left(a_{i} \mid h_{i}\right)>0$ ) is rationalizable for the type (i.e., $a_{i} \in R_{i}^{\infty}\left(h_{i}\right)$ ).

To assess the robustness of equilibrium predictions, we again take the perspective of a researcher who can observe finitely many orders of beliefs with some noise. That is, there is some finite order $\kappa$ and some $\eta>0$ such that for each $\ell<\kappa$, the researcher cannot rule out $\ell$ th-order beliefs that are within $\eta$ of the observed $\ell$ th-order belief (in the usual weak topology). ${ }^{19}$ In particular, if the researcher observes that a player has an $m$ th-order belief, then he rules out that the player has a depth of reasoning strictly less than $m .^{20}$ We write $O_{\eta, \kappa}\left(t_{i}\right)$ for the set of types that the researcher finds possible if he observes the type $t_{i}$. We focus on the case where the noise is small, that is, $\eta \rightarrow 0$ and $\kappa \rightarrow \infty$.

Definition 3. A pair $\left(M^{\prime}, \tau\right)$ is an $(\eta, \kappa)$-perturbation of a model $M=(\widetilde{\Theta}, T)$ if $M^{\prime}=$ $\left(\widetilde{\Theta}^{\prime}, T^{\prime}\right)$ is a finite model and $\tau: T \rightarrow T^{\prime}$ is such that $\tau_{i}\left(t_{i}\right) \in O_{\eta, \kappa}\left(t_{i}\right)$ for every $t_{i} \in T_{i}$.

This naturally leads to the following robustness requirement: since a researcher cannot rule out any $(\eta, \kappa)$ perturbation of a model, a prediction for a model is robust if it is

\footnotetext{
${ }^{19}$ In addition, a researcher may want to measure a type's signal $x_{i}$. We abstract away from this for the purposes of this section; so we take $X_{i}=\left\{x_{i}\right\}$ in this section.

${ }^{20}$ More precisely, the topology on the set of $m$ th-order beliefs is the usual weak topology. This topology can be metrized with the Prokhorov metric $d_{P}^{m}$, and we say that a type with $m$ th-order belief $\mu_{i}^{m}$ is within $\eta$ of a type with $m$ th-order belief $\nu_{i}^{m}$ if $d_{P}^{m}\left(\mu_{i}^{m}, \nu_{i}^{m}\right)<\eta$. If $\eta$ is not too large (e.g., $\left.\eta<1\right)$, then the researcher can distinguish between types with different depths of reasoning $k, k^{\prime} \leq \kappa$, as well as between a type that has depth $k \leq \kappa$ and a type with depth $k^{\prime}>\kappa$.
} 
valid for any $(\eta, \kappa)$ perturbation of the model. In the context of Bayesian-Nash equilibrium, this gives the following condition. ${ }^{21}$

Definition 4. A Bayesian-Nash equilibrium $\sigma$ for $M=(\widetilde{\Theta}, T)$ is $(\eta, \kappa)$-robust if, for every $(\eta, \kappa)$ perturbation $\left(M^{\prime}, \tau\right)$ of $M$, there is a Bayesian-Nash equilibrium $\sigma^{\prime}$ for $M^{\prime}$ that coincides with $\sigma$ on $\tau(T)$ (i.e., for each player $i=1$, 2, type $h_{i} \in T_{i}, \sigma_{i}^{\prime}\left(\tau_{i}\left(h_{i}\right)\right)=$ $\left.\sigma_{i}\left(h_{i}\right)\right)$. A Bayesian-Nash equilibrium is robust if it is $(\eta, \kappa)$-robust for some $\eta>0$ and $\kappa<\infty$.

Equilibrium may provide stronger predictions than rationalizability in models with multiplicity, that is, models that have a type with multiple rationalizable actions. However, there may be tension between the desire to get sharp prediction and the requirement that predictions be robust. The next result shows that if there is common belief in an infinite depth, then any equilibrium that makes stronger predictions than rationalizability is not robust.

Proposition 6 (No robust refinement under common belief in infinite depth; Weinstein and Yildiz 2007). Under Assumption R-Dom, if $M=(\widetilde{\Theta}, T)$ is a finite model with multiplicity that is consistent with common belief in an infinite depth (i.e., $T \subset C^{\infty}$ ) and $\widetilde{\Theta}$ is finite, then $M$ does not have a robust equilibrium.

Proposition 6 implies that if it is assumed that there is common belief in an infinite depth, then a researcher who is concerned with the robustness of his predictions cannot make sharper predictions by using a refinement of rationalizability. In the next section, we show that the situation is strikingly different when there is almost-common belief in an infinite depth.

\subsection{Results}

If we relax the assumption that there is common belief in an infinite depth, then robust refinements exist. To state the result, say that a model has multiplicity if it has a type with multiple rationalizable actions.

Proposition 7 (Robustness of strict equilibrium under almost-common belief in infinite depth). Under Assumption R-Mult $\left(A^{\prime}\right)$, for every $m=1,2, \ldots$, there is a model $M$ with multiplicity and $m$ th-order belief in an infinite depth such that every strict BayesianNash equilibrium for $M$ is robust.

Since equilibrium is a refinement of rationalizability and there are types with multiple rationalizable actions, Proposition 7 immediately implies that robust refinements of rationalizability exist.

\footnotetext{
${ }^{21}$ The robustness requirement in Definition 4 is stronger than the robustness requirement in Weinstein and Yildiz (2007). First, they do not require that the strategy profile for the perturbed model coincides exactly with the strategy profile on the original model, only that it produces the same behavioral patterns. Also, their definition of an $(\eta, \kappa)$ perturbation $\left(M^{\prime}, \tau\right)$ requires $M^{\prime}$ to admit a full support common prior. Working with a stronger robustness requirement strengthens our positive result.
} 
Again, the contrast between the case with common belief in an infinite depth and almost-common belief in an infinite depth is stark. If there is common belief in an infinite depth, then the requirement that predictions be robust eliminates all equilibria if there is a type with multiple rationalizable actions (Proposition 6). In contrast, if we relax the assumption that there is common belief in an infinite depth, then there are models for which every strict equilibrium is robust (Proposition 7). In that case, robustness does not impose any additional restrictions on equilibria beyond strictness.

Proposition 7 is not a simple corollary of the results for rationalizability. In the case of rationalizability, the key is to ensure that players can hold multiple conjectures about the opponent's behavior. In the case of equilibrium, players have a single conjecture that coincides with the opponent's actual strategy. The role of finite-depth types differs correspondingly. In the case of equilibrium, finite-depth types do not act as a grain of multiplicity, as in the case of rationalizability. Rather, their role is to "anchor" the behavior of high-depth types. Anchoring the behavior of types is critical: in environments with common belief in an infinite depth of reasoning, the high-order beliefs of types can vary arbitrarily. But, if there is only almost-common belief in an infinite depth, then behavior can be determined at a finite order. To see this, consider an infinite-depth type that assigns high probability (say, $1-\eta$ for $\eta>0$ small) to types of depth at most $k<\infty$. Then, since most of the probability mass is concentrated on types whose higher-order beliefs cannot be varied arbitrarily, perturbations of high-order beliefs have only a limited impact: the robustness of equilibrium behavior for the finite-depth types carries over to the infinite-depth types.

The proof of Proposition 7 illustrates the difference between the arguments for the case where types have a strictly dominant action and where they have multiple rationalizable actions. In the former case, strategic beliefs (i.e., beliefs about others' actions, beliefs about others' beliefs about actions, and so on) are irrelevant, and it follows directly that Bayesian-Nash equilibrium is robust to small perturbation of beliefs, even if there is common belief in an infinite depth. By contrast, if types have multiple best responses, a player's best response depends on his conjecture about the opponent's action. In this case, strategic beliefs matter and establishing robustness requires considerable care. In particular, it requires relaxing the assumption that there is common belief in an infinite depth so that finite-depth types, whose equilibrium actions do not depend on their high-order beliefs about strategies, can be used to anchor the behavior of types with a higher depth of reasoning.

\subsection{Example: Global games}

As noted above, robustness does not put much restrictions on equilibrium behavior. The global games introduced by Carlsson and van Damme (1993) provide a particularly clear illustration of this point. In global games, players play a supermodular game at each state $\theta$. Each player $i$ has two actions, labeled $\tilde{a}$ and $\tilde{b}$. Each action $a_{i}=\tilde{a}, \tilde{b}$ is strictly dominant for player $i$ at some state $\theta^{\tilde{a}_{i}}$, and for some $\theta^{*}$, the complete-information game (with payoff functions $u_{i}\left(\cdot, \cdot, \theta^{*}\right): A \rightarrow \mathbb{R}$ ) has two strict Nash equilibria, $(\tilde{a}, \tilde{a})$ and $(\tilde{b}, \tilde{b})$ (say). Clearly, every global game satisfies Assumptions R-Dom and R-Mult $\left(A^{\prime}\right)$ (with 
$\left.A_{i}^{\prime}=\{\tilde{a}, \tilde{b}\}\right)$. As in much of the applied literature, we assume that payoff functions are symmetric. Example 1 is an example of a symmetric global game.

The literature focuses on the question of which equilibria of the completeinformation game are robust to the introduction of a small amount of incomplete information about payoffs. The next result shows that if there is almost-common belief in an infinite depth, the requirement that predictions be robust need not rule out any of the equilibria of the complete-information game.

Proposition 8 (Robust equilibria in global games under almost-common belief in an infinite depth). Fix a symmetric global game. For every $m=1,2, \ldots$, there is a finite model $M$ with types with mth-order belief in an infinite depth, and robust equilibria $\sigma^{\tilde{a}}$ and $\sigma^{\tilde{b}}$ for $M$ such that if all types believe that the game has multiple Nash equilibria (i.e., $\left.\theta=\theta^{*}\right)$, then players play according to the Nash equilibrium $(\tilde{a}, \tilde{a})$ under $\sigma^{\tilde{a}}$, while they play according to the Nash equilibrium on $(\tilde{b}, \tilde{b})$ under $\sigma^{\tilde{b}}$.

Proposition 8 shows that if we relax the strong assumption that there is common belief in an infinite depth, then there are models with complete information about payoffs in which every Nash equilibrium is robust to the introduction of uncertainty about payoffs. This contrasts with the limit case with common belief in an infinite depth. In that case, if the complete-information game has multiple rationalizable actions, then the researcher is unable to make any robust predictions (Propositions 3 and 6).

\section{Related literature}

\section{Almost-common belief in rationality}

In the context of repeated games, Kreps et al. (1982), Kreps and Wilson (1982), and Milgrom and Roberts (1982) show that a grain of doubt about players' rationality can have a large impact on behavior. We likewise introduce a grain of doubt about the opponent's reasoning ability. However, there is a fundamental difference between their "irrational" types and our "naive," nonstrategic, types. While irrational types are (commonly known to be) committed to playing a certain action, we follow the level- $k$ literature in assuming that nonstrategic types can play any action. Thus, in a sense, our approach can be viewed as relaxing common-knowledge assumptions about the behavior of players who are not fully sophisticated. This requires a novel approach. While in the existing literature, the commitment of irrational types to a particular course of action renders high-order reasoning about behavior irrelevant, in our setting, players must entertain different conjectures about their opponent's behavior. This requires them to reason about their opponent's high-order beliefs. Accordingly, unlike in the existing literature, our results require an approach that is inherently strategic in nature. In particular, in our model, the assumption that there is a small amount of uncertainty about players' reasoning ability cannot be replaced by the assumption that there is a small amount of uncertainty about payoffs, unlike in the existing literature. 
Finite depth of reasoning in Bayesian games

A growing literature studies the behavior of players with a finite depth of reasoning in games with incomplete information. The experimental literature studies behavior in a wide range of games ranging from auctions to betting and market entry games (see Crawford et al. 2013, for a survey). This paper is the first to study the robustness of predictions to perturbations of beliefs about payoffs and reasoning ability. Strzalecki (2014) shows that the risk-dominant solution may not be uniquely selected in the electronicmail game of Rubinstein (1989), but he does not consider the robustness of predictions. ${ }^{22}$ In fact, it is not possible to study the robustness of predictions in his framework since it does not allow for perturbations of beliefs about payoffs. ${ }^{23}$ Kets $(2012,2013)$ introduces a class of type spaces for players with an arbitrary depth of reasoning and defines rationalizability and Bayesian-Nash equilibrium for her setting. An important difference is that Kets's solution concepts are refinements of the corresponding solution concepts for the standard case with common belief in an infinite depth.

\section{Appendix A: Type SPACES}

Belief hierarchies provide an explicit description of players' higher-order beliefs. Higherorder beliefs can also be described implicitly, using a type space (cf. Harsanyi 1967). Here we define type spaces that generate belief hierarchies with an arbitrary (finite or infinite) depth. Type spaces are defined for a given set $\Theta$ of states of nature (taken to be compact metric, as before).

Definition 5. A type space is a tuple

$$
\mathcal{T}:=\left\langle\left(T_{i}\right)_{i=1,2},\left(\beta_{i}\right)_{i=1,2},\left(\chi_{i}\right)_{i=1,2}\right\rangle,
$$

where for each player $i, T_{i}=T_{i}^{\infty} \cup \bigcup_{\ell=0}^{\infty} T_{i}^{\ell}$ is the set of types for player $i$, assumed to be nonempty and Polish, and $\chi_{i}$ is a continuous function that maps each type $t_{i} \in T_{i}$ into a signal $\chi_{i}\left(t_{i}\right) \in X_{i}$. The function $\beta_{i}$ maps types into beliefs: for $t_{i} \in T_{i}^{k}, k \leq \infty$, $\beta_{i}\left(t_{i}\right):=\beta_{i}^{k}\left(t_{i}\right)$, where

- $\beta_{i}^{0}$ maps each $t_{i} \in T_{i}^{0}$ into $h_{i}^{*, 0}$,

- $\beta_{i}^{k}$ is continuous and maps each $t_{i} \in T_{i}^{k}$ into a belief in $\Delta\left(\Theta \times T_{-i}^{\leq k-1}\right)$, where $T_{-i}^{\leq k}:=\bigcup_{\ell=0}^{k} T_{-i}^{\ell}$,

- $\beta_{i}^{\infty}$ is continuous and maps each $t_{i} \in T_{i}^{\infty}$ into a belief in $\Delta\left(\Theta \times T_{-i}\right)$.

If there is $t_{i} \in T_{i}^{k}$ for $k=1,2, \ldots$, then there is a type $t_{-i} \in T_{-i}^{m}$ for some $m<k .^{24}$

\footnotetext{
${ }^{22}$ Murayama (2015) studies the robustness of Nash equilibria in level- $k$ and cognitive hierarchy models when there is uncertainty about the behavior of nonstrategic players. He does not consider uncertainty about payoffs, and his robustness notion does not involve almost-common belief.

${ }^{23}$ In addition, his universal type space does not contain the universal type space for standard types of Mertens and Zamir (1985), so that it is not possible to consider arbitrarily small deviations from standard assumptions in his framework.

${ }^{24}$ This ensures that types' beliefs are well defined (i.e., have nonempty support).
} 
Thus, each type in $T_{i}^{0}$ is associated with the "naive" type $h_{i}^{*, 0}$. Types in $T_{i}^{k}, k<\infty$, are mapped into a belief over the types with a strictly lower index, and types in $T_{i}^{\infty}$ have a belief over types with any index. As before, a type's signal $\chi_{i}\left(t_{i}\right) \in X_{i}$ represents its (payoff-irrelevant) private information. A standard (Harsanyi) type space is simply a type space in which every type has index $k=\infty$. The Supplemental Appendix shows that each type can be mapped into a belief hierarchy in the usual way, and that a type with index $k$ corresponds to a belief hierarchy of depth $k$.

We can define the set of rationalizable actions for a type in the same way as before. The only difference is that the conjectures in the definition of the set $R_{i}^{T, k}$ of $k$ rationalizable actions are now (measurable) functions from $\Theta \times T_{-i}$ into $\Delta\left(A_{-i}\right)$. As we show now, the set of $(k-)$ rationalizable actions for a type depends only on its belief hierarchy, as in the standard case (Dekel et al. 2007, Lemma 1). To state the result, we need some more definitions. In the Supplemental Appendix, we define a function $h_{i}^{T}$ that maps each type into its (full) belief hierarchy. We then have the following lemma.

Lemma 7. For any type $t_{i} \in T_{i}$ and every $k=1,2, \ldots$,

$$
R_{i}^{T, k}\left(t_{i}\right)=R_{i}^{k}\left(h_{i}^{T}\left(t_{i}\right)\right) .
$$

Hence,

$$
R_{i}^{T, \infty}\left(t_{i}\right)=R_{i}^{\infty}\left(h_{i}^{T}\left(t_{i}\right)\right)
$$

Lemma 7 implies that studying the rationalizable actions of players within the context of the universal type space is without loss of generality.

\section{ApPendix B: The Universal type SPACE}

Following Mertens and Zamir (1985), we use the set of all belief hierarchies to construct the universal type space. The set of types for player $i$ is the set $H_{i}$ of belief hierarchies. The next result implies that $H_{i}$ is well defined.

Lemma 8. For every $k=1,2, \ldots, \infty$, the set $H_{i}^{k}$ is nonempty and compact metric. Hence, $H_{i}$ is well defined and Polish.

The next result shows that each belief hierarchy specifies a belief about the full hierarchy of other players, not just about the individual levels of the hierarchy.

Lemma 9. (a) For each belief hierarchy $h_{i}=\left(x_{i}, \mu_{i}^{0}, \mu_{i}^{1}, \ldots\right) \in H_{i}^{\infty}$, there exists a unique Borel probability measure $\mu_{i}\left(h_{i}\right)$ on $\Theta \times H_{-i}$ such that

$$
\operatorname{marg}_{\widetilde{\Omega}_{i}^{\ell-1}} \mu_{i}\left(h_{i}\right)=\mu_{i}^{\ell}
$$

for all $\ell=1,2, \ldots$ 
(b) For each $k>0$ and every belief hierarchy $h_{i}=\left(x_{i}, \mu_{i}^{0}, \mu_{i}^{1}, \ldots, \mu_{i}^{k}\right) \in H_{i}^{k}$, there exists a unique Borel probability measure $\mu_{i}\left(h_{i}\right)$ on $\Theta \times H_{-i}^{\leq k-1}$ such that

$$
\operatorname{marg}_{\widetilde{\Omega}_{i}^{\ell-1}} \mu_{i}\left(h_{i}\right)=\mu_{i}^{\ell}
$$

for all $\ell=1, \ldots, k$.

Thus, each belief hierarchy of player $i$ can be associated with a belief over the set $\Theta$ of states of nature, the signal spaces $X_{-i}$, and over the other players' belief hierarchies, in such a way that $i$ 's belief over her $\ell$ th-order space of uncertainty coincides with his $\ell$ th-order belief as specified by her hierarchy of beliefs. That is, the construction is canonical.

Using Lemma 9, we can construct a function that assigns to each belief hierarchy $h_{i}$ its signal (by projecting $h_{i}$ onto $X_{i}$ ) and a belief about nature and other players' hierarchies (as given by Lemma 9). The inverse of this function assigns to each signal-belief pair $\left(x_{i}, \mu_{i}\right) \in X_{i} \times \Delta\left(\Theta \times H_{-i}\right)$ the associated belief hierarchy (possibly finite). Proposition 9 shows that these functions are continuous. Thus, for every depth $k$, we have a homeomorphism.

Proposition 9. There is a homeomorphism $\tilde{\psi}_{i}^{\infty}: H_{i}^{\infty} \rightarrow X_{i} \times \Delta\left(\Theta \times H_{-i}\right)$. Moreover, for each $k=1,2, \ldots$, there is a homeomorphism $\tilde{\psi}_{i}^{k}: H_{i}^{k} \rightarrow X_{i} \times \Delta\left(\Theta \times H_{-i}^{\leq k-1}\right)$.

We use this result to define the universal type space. Write $\psi_{i}^{k}\left(h_{i}\right), k=1,2, \ldots$, $h_{i} \in H_{i}^{k}$, for the projection of $\tilde{\psi}_{i}^{k}$ into $\Delta\left(\Theta \times H_{-i}^{\leq k-1}\right)$; likewise, $\psi_{i}^{\infty}\left(h_{i}\right), h_{i} \in H_{i}^{\infty}$, is the projection of $\tilde{\psi}_{i}^{\infty}\left(h_{i}\right)$ into $\Delta\left(\Theta \times H_{-i}\right)$. Define $\psi_{i}^{0}: H_{i}^{0} \rightarrow\left\{h_{i}^{*, 0}\right\}$ in the obvious way, and view $\psi_{i}^{k}\left(h_{i}\right), h_{i} \in H_{i}^{k}, k<\infty$, as a probability measure on $\Delta\left(\Theta \times H_{-i}\right)$. Let $\psi_{i}: H_{i} \rightarrow \Delta\left(\Theta \times H_{-i}\right)$ be the function that coincides with $\psi_{i}^{k}$ on $H_{i}^{k}$. Thus, $\psi_{i}$ is continuous. Finally, let $\chi_{i}^{*}\left(x_{i}, \mu_{i}^{0}, \mu_{i}^{1}, \ldots\right)=x_{i}$. Then $\mathcal{T}^{*}:=\left\langle\left(H_{i}\right)_{i=1,2},\left(\psi_{i}\right)_{i=1,2},\left(\chi_{i}^{*}\right)_{i=1,2}\right\rangle$ is a type space. This type space is universal in the sense that it generates all belief hierarchies; see the Supplemental Appendix for a proof. A useful property is that the universal type space is complete in the sense of Brandenburger (2003): for every belief $\nu_{i} \in \Delta\left(\Theta \times H_{-i}\right)$, there is a type $h_{i} \in H_{i}$ with $\psi_{h_{i}}=\nu_{i}$.

Remark 2. Types with different depths of reasoning can look very similar, yet correspond to different beliefs. For example, consider a depth- $k$ type $h_{i}^{k}$ and a depth- $m$ type $h_{i}^{m}$ for $m<k$ that have the same beliefs about $\Theta$ and that both believe (i.e., assign probability 1 to the event) that the opponent has a given depth- $(m-1)$ type $h_{-i}^{m-1}$. These beliefs look very similar, yet they are different: the belief $\psi_{h_{i}^{k}}$ is a probability measure defined on the set $\bigcup_{\ell \leq k-1} H_{-i}^{\ell}$ of types of depth at most $k-1$, while the belief $\psi_{h_{i}^{m}}$ is a probability measure defined on the set $\bigcup_{\ell \leq m-1} H_{-i}^{\ell}$ of types of depth at most $m-1$. While the distinction between such types can be conceptually important (cf. Heifetz et al. 2013), our results do not depend on it in any way. 


\section{APPENDix C: Transfinite ELIMINATION PROCESS}

We show that rationalizability satisfies a best-reply property: Any rationalizable action for a type is a best response against the belief that the opponent plays a rationalizable strategy, as in the standard case (Dekel et al. 2007, Proposition 4). We use this to show that the set of rationalizable actions cannot be refined further if we perform more rounds of elimination (Proposition 10 below).

LeMma 10. For every $h_{i} \in H_{i}$ and $a_{i} \in R_{i}^{\infty}\left(h_{i}\right)$, there is a measurable conjecture $s_{-i}: \Theta \times$ $H_{-i} \rightarrow \Delta\left(A_{-i}\right)$ such that $\operatorname{supp} s_{-i}\left(\theta, h_{-i}\right) \subset R_{-i}^{\infty}\left(h_{-i}\right)$ for all $\theta, h_{-i}$, and

$$
a_{i} \in \arg \max \max _{a_{i}^{\prime} \in A_{i}} \int_{\Theta \times H_{-i} \times A_{-i}} u_{i}\left(a_{i}^{\prime}, a_{-i}, \theta\right) s_{-i}\left(\theta, h_{-i}\right)\left(a_{-i}\right) \mathrm{d} \psi_{h_{i}} .
$$

Proof. Fix $h_{i} \in H_{i}$ and $a_{i} \in R_{i}^{\infty}\left(h_{i}\right)$. The result follows directly if $h_{i}$ has finite depth. So suppose that $h_{i}$ has an infinite depth of reasoning. For every $m$, there exists $s_{-i}^{m}: \Theta \times H_{-i} \rightarrow \Delta\left(A_{-i}\right)$ such that $\operatorname{supp} s_{-i}^{m}\left(\theta, h_{-i}\right) \subset R_{-i}^{m-1}\left(h_{-i}\right)$ for all $\theta, h_{-i}$, and $a_{i} \in \arg \max _{\tilde{a}_{i}} \int u_{i}\left(\tilde{a}_{i}, s_{-i}^{m}\left(\theta, h_{-i}\right), \theta\right) \mathrm{d} \psi_{h_{i}}$. We need to show that there is $s_{-i}: \Theta \times$ $H_{-i} \rightarrow \Delta\left(A_{-i}\right)$ such that $\operatorname{supp} s_{-i}\left(\theta, h_{-i}\right) \subset R_{-i}^{\infty}\left(h_{-i}\right)$ for all $\theta, h_{-i}$, and $a_{i} \in$ $\arg \max _{\tilde{a}_{i}} \int u_{i}\left(\tilde{a}_{i}, s_{-i}\left(\theta, h_{-i}\right), \theta\right) \mathrm{d} \psi_{h_{i}}$. Since the set of functions from $\Theta \times H_{-i}$ to $\Delta\left(A_{-i}\right)$ is compact Hausdorff, the sequence $\left\{s_{-i}^{m}\right\}_{m}$ has a convergent subsequence $\left\{s_{-i}^{m_{k}}\right\}_{k}$, and the (pointwise) limit $s_{-i}:=\lim _{k \rightarrow \infty} s_{-i}^{m_{k}}$ is unique. Moreover, $s_{-i}$ is measurable (Aliprantis and Border 2006, Lemma 4.29). By construction, supp $s_{-i}\left(\theta, h_{-i}\right) \subset R_{-i}^{\infty}\left(h_{-i}\right)$ for all $\theta, h_{-i}$. By the dominated convergence theorem, $\int u_{i}\left(\tilde{a}_{i}, s_{-i}^{m_{k}}\left(\theta, h_{-i}\right), \theta\right) \mathrm{d} \psi_{h_{i}}$ converges to $\int u_{i}\left(\tilde{a}_{i}, s_{-i}\left(\theta, h_{-i}\right), \theta\right) \mathrm{d} \psi_{h_{i}}$ for $\tilde{a}_{i} \in A_{i}$. Hence, $a_{i} \in \arg \max _{\tilde{a}_{i}} u_{i}\left(\tilde{a}_{i}, s_{-i}^{m_{k}}\left(\theta, h_{-i}\right), \theta\right) \mathrm{d} \psi_{h_{i}}$.

We can use this to show that continuing the elimination process does not eliminate any additional strategies. Following Lipman (1994), we define a rationalizability concept based on the transfinite elimination of strictly dominated strategies. This requires working with ordinals. Recall that an ordinal $\alpha$ can be identified with the set $\{\beta: \beta<\alpha\}$ of its predecessors; we identify the finite ordinals with the natural numbers $0,1,2, \ldots$, so that the first infinite ordinal $\omega$ is equal to $\{0,1, \ldots\}=\mathbb{N}$. The successor of an ordinal $\alpha$ is the least ordinal greater than $\alpha$. An ordinal is a successor ordinal if it is the successor of some ordinal. An ordinal is a limit ordinal if it is not 0 or a successor ordinal.

Let $\mathcal{R}_{i}^{0}=R_{i}^{0}$. For $\alpha>0$, suppose $\mathcal{R}_{i}^{\gamma}$ has been defined for all $\gamma<\alpha$. If $\alpha$ is the successor of some ordinal $\beta$, then the definition of $\mathcal{R}_{i}^{\alpha}$ is similar to before:

$$
\mathcal{R}_{i}^{\alpha}\left(h_{i}\right):=\left\{\begin{array}{c}
\text { there is a measurable } s_{-i}: \Theta \times H_{-i} \rightarrow \Delta\left(A_{-i}\right) \text { s.t. } \\
a_{i} \in A_{i}: \operatorname{supp} s_{-i}\left(\theta, h_{-i}\right) \subseteq \mathcal{R}_{-i}^{\beta}\left(h_{-i}\right) \text { for all } h_{-i} \in H_{-i}, \theta \in \Theta ; \text { and } \\
a_{i} \in \arg \max _{a_{i}^{\prime} \in A_{i}} \int_{\Theta \times H_{-i} \times A_{-i}} u_{i}\left(a_{i}^{\prime}, a_{-i}, \theta\right) s_{-i}\left(\theta, h_{-i}\right)\left(a_{-i}\right) \mathrm{d} \psi_{h_{i}}
\end{array}\right\} .
$$

If $\alpha$ is a limit ordinal, then define $\mathcal{R}_{i}^{\alpha}$ by

$$
\mathcal{R}_{i}^{\alpha}\left(h_{i}\right):=\bigcap_{\gamma<\alpha} \mathcal{R}_{i}^{\gamma}\left(h_{i}\right) .
$$


Then, for finite $\alpha, \mathcal{R}_{i}^{\alpha}\left(h_{i}\right)=R_{i}^{\alpha}\left(h_{i}\right)$. Moreover, $\mathcal{R}_{i}^{\omega}\left(h_{i}\right)=R_{i}^{\infty}\left(h_{i}\right)$. As we iterate beyond $\omega$, the set of rationalizable actions may continue to shrink. It turns out, though, that performing transfinitely many rounds of elimination of strictly dominated strategies does not eliminate any additional actions.

Proposition 10. For any ordinal $\alpha \geq \omega$ and any $h_{i} \in H_{i}, \mathcal{R}_{i}^{\alpha}\left(h_{i}\right)=R_{i}^{\infty}\left(h_{i}\right)$.

Proof. By Lemma 10, $\mathcal{R}_{i}^{\omega+1}\left(h_{i}\right)=R_{i}^{\infty}\left(h_{i}\right)$.

\section{Appendix D: Proofs}

\section{D.1 Proof of Lemma 1}

The result follows from a simple induction. For $h_{i} \in H_{i}^{0}, R_{i}^{0}\left(h_{i}\right)=R_{i}^{1}\left(h_{i}\right)=\cdots=R_{i}^{\infty}\left(h_{i}\right)$. For $m>0$, suppose that for $n \leq m-1, h_{i} \in H_{i}^{n}, R_{i}^{n}\left(h_{i}\right)=R_{i}^{n+1}\left(h_{i}\right)=\cdots=R_{i}^{\infty}\left(h_{i}\right)$. Then, for any $h_{i} \in H_{i}^{m}, R_{i}^{m}\left(h_{i}\right)=R_{i}^{m+1}\left(h_{i}\right)=\cdots=R_{i}^{\infty}\left(h_{i}\right)$, as $\psi_{h_{i}}$ has support in $H_{-i}^{0} \cup \cdots \cup$ $H_{-i}^{m-1}$.

\section{D.2 Proof of Lemma 2}

The proof of Lemma 2 below is a straightforward adaptation of the proof of Lemma 3 in Yildiz (2004). Lemma 3 of Yildiz (2004) extends Lemma 1 of Dekel et al. (2007) to the case where $\Theta$ is compact metric (rather than finite, as in Dekel et al.). The only significant difference between Yildiz's setting and ours is that the set $H_{i}$ of belief hierarchies of arbitrary depth satisfies weaker topological conditions than the set of standard belief hierarchies that Yildiz considers: the set $H_{i}$ is Polish, while the set of standard belief hierarchies is compact metric. We therefore adapt Yildiz's proof so that it makes reference only to the set of finite-order belief hierarchies, which satisfy the same topological conditions as in the standard case.

We define a version of $m$-rationalizability that is a function only of players' $m$ th-order belief hierarchies, and then use it to prove the results for $R_{i}^{m}$ (whose domain is the set $H_{i}$ of belief hierarchies). We need some more notation. For $m=0,1, \ldots$, define $G_{i}^{m}:=H_{i}^{m} \cup$ $\widetilde{H}_{i}^{m}$ to be the set of $m$ th-order belief hierarchies. Also, define $\tilde{G}_{i}^{m}:=H_{i}^{0} \cup \cdots \cup H_{i}^{m-1} \cup G_{i}^{m}$. For an $m$ th-order belief hierarchy $g_{i}^{m}=\left(x_{i}, \mu_{i}^{0}, \ldots, \mu_{i}^{m}\right)$, write $\nu_{g_{i}^{m}}^{m}:=\mu_{i}^{m}$ for its induced $m$ th-order belief. Note that $\nu_{g_{i}^{m}}^{m}$ is a belief on $\Theta \times \tilde{G}_{i}^{m-1}$. For $g_{i} \in \tilde{G}_{i}^{m}$, define $n_{g_{i}}=k$ if $g_{i} \in H_{i}^{k}$ and $n_{g_{i}}=m$ if $g_{i} \in \widetilde{H}_{i}^{m}$.

For $g_{i}^{0} \in G_{i}^{0}$, let $\widetilde{R}_{i}^{0}\left(g_{i}^{0}\right):=A_{i}$. For $m>0$, suppose that $\widetilde{R}_{i}^{m-1}: G_{i}^{m-1} \rightarrow A_{i}$ has been defined and define the correspondence $\widetilde{R}_{i}^{m}: G_{i}^{m} \rightarrow A_{i}$ by

$\widetilde{R}_{i}^{m}\left(g_{i}^{m}\right):=\left\{\begin{array}{c}\text { there is a measurable } s_{-i}: \Theta \times \tilde{G}_{-i}^{m-1} \rightarrow \Delta\left(A_{-i}\right) \text { s.t. } \\ a_{i} \in A_{i}: \operatorname{supp} s_{-i}\left(\theta, g_{-i}\right) \subseteq \widetilde{R}_{-i}^{n_{i}}\left(g_{-i}\right) \text { for all } g_{-i} \in \tilde{G}_{-i}^{m-1}, \theta \in \Theta ; \text { and } \\ a_{i} \in \arg \max _{a_{i}^{\prime} \in A_{i}} \int_{\Theta \times \tilde{G}_{-i}^{m-1} \times A_{-i}} u_{i}\left(a_{i}^{\prime}, a_{-i}, \theta\right) s_{-i}\left(\theta, g_{-i}\right)\left(a_{-i}\right) \mathrm{d} \nu_{g_{i}^{m}}^{m} .\end{array}\right\}$. 
We show that $\widetilde{R}_{i}^{m}$ is upper hemicontinuous. ${ }^{25}$ We then use this to prove that $R_{i}^{m}$ is upper hemicontinuous for $m \leq \infty$.

LEMMA 11. The correspondence $\widetilde{R}_{i}^{m}$ is upper hemicontinuous and has nonempty values.

Proof. By Theorem 17.11 of Aliprantis and Border (2006), $\widetilde{R}_{i}^{m}$ is upper hemicontinuous if and only if it has a closed graph, where the graph of a correspondence $F$ : $X \rightarrow Y$ is $\operatorname{Gr}(F)=\{(x, y) \in X \times Y: y \in F(x)\}$. By definition, $\operatorname{Gr}\left(\widetilde{R}_{i}^{0}\right)=G_{i}^{0} \times A_{i}$. It follows immediately that the correspondence $\widetilde{R}_{i}^{0}$ is nonempty-valued and upper hemicontinuous. For $m>0$, suppose that $\widetilde{R}_{i}^{n}$ is upper hemicontinuous and nonemptyvalued for $n \leq m-1$. We claim that $\operatorname{Gr}\left(\widetilde{R}_{i}^{m}\right)$ is closed. By the induction hypothesis, $\Theta \times \bigcup_{n \leq m-1} \operatorname{Gr}\left(\widetilde{R}_{-i}^{n}\right) \subset \Theta \times \tilde{G}_{-i}^{m-1} \times A_{-i}$ is closed and nonempty. Since $\Theta \times \tilde{G}_{-i}^{m-1} \times A_{-i}$ is compact, so is $\Theta \times \bigcup_{n \leq m-1} \operatorname{Gr}\left(\widetilde{R}_{-i}^{n}\right)$. Hence, $\Delta\left(\Theta \times \bigcup_{n \leq m-1} \operatorname{Gr}\left(\widetilde{R}_{-i}^{n}\right)\right)$ is compact. Moreover, since $u_{i}$ is continuous and bounded (being defined on a compact space), $\int u_{i}\left(\cdot, a_{-i}, \theta\right) \mathrm{d} \nu_{i}^{m}$ is a continuous function of $\nu_{i}^{m} \in \Delta\left(\Theta \times \bigcup_{n \leq m-1} \operatorname{Gr}\left(\widetilde{R}_{-i}^{n}\right)\right)$ to $\mathbb{R}$. Define $\mathcal{M}_{i}^{m}: \Delta\left(\Theta \times \bigcup_{n \leq m-1} \operatorname{Gr}\left(\widetilde{R}_{-i}^{n}\right)\right) \rightarrow A_{i}$ by

$$
\mathcal{M}_{i}^{m}\left(\nu_{i}^{m}\right):=\underset{\tilde{a}_{i} \in A_{i}}{\arg \max } \int u_{i}\left(\tilde{a}_{i}, a_{-i}, \theta\right) \mathrm{d} \nu_{i}^{m} .
$$

By the Berge maximum theorem, $\mathcal{M}_{i}^{m}\left(\nu_{i}^{m}\right)$ is nonempty, and $\operatorname{Gr}\left(\mathcal{M}_{i}^{m}\right)$ is closed and thus compact in $\Delta\left(\Theta \times \bigcup_{n \leq m-1} \operatorname{Gr}\left(\widetilde{R}_{-i}^{n}\right)\right) \times A_{i}$. Fix $\nu_{i}^{m} \in \Delta\left(\Theta \times \bigcup_{n \leq m-1} \operatorname{Gr}\left(\widetilde{R}_{-i}^{n}\right)\right)$. If $\nu_{i}^{m}$ has support in $\Theta \times\left(H_{-i}^{0} \cup \cdots \cup H_{-i}^{m-1}\right) \subsetneq \Theta \times \tilde{G}_{-i}^{m-1}$, then there exist unique $g_{i}^{m} \in H_{i}^{m}$ and $\tilde{g}_{i}^{m} \in \widetilde{H}_{i}^{m}$ such that the marginal of $\nu_{i}^{m}$ on $\Theta$ and the other player's $(m-1)$ th-order belief hierarchy coincides with the $m$ th-order belief induced by $g_{i}^{m}$ and $\tilde{g}_{i}^{m}$. Otherwise (if $\nu_{i}^{m}$ has support in $\left.\Theta \times\left(H_{-i}^{0} \cup \cdots \cup H_{-i}^{m-1} \cup \widetilde{H}_{-i}^{m-1}\right)=\Theta \times \tilde{G}_{-i}^{m-1}\right), \tilde{g}_{i}^{m}$ is the unique $m$ th-order belief hierarchy in $G_{i}^{m}$ such that the induced $m$ th-order belief coincides with the marginal of $\nu_{i}^{m}$. Let $\varphi_{i}^{m}$ and $\tilde{\varphi}_{i}^{m}$ be the functions that map $\left(a_{i}, \nu_{i}^{m}\right)$ into $\left(a_{i}, g_{i}^{m}\right)$ and $\left(a_{i}, \tilde{g}_{i}^{m}\right)$, respectively (if the former exist). Then $\varphi_{i}^{m}$ and $\tilde{\varphi}_{i}^{m}$ are continuous (on the appropriate domain and range spaces), and $\operatorname{Gr}\left(\widetilde{R}_{i}^{m}\right)=\varphi_{i}\left(\mathcal{M}_{i}^{m}\right) \cup \tilde{\varphi}_{i}^{m}\left(\mathcal{M}_{i}^{m}\right)$. Then $\operatorname{Gr}\left(\widetilde{R}_{i}^{m}\right)$ is closed and $\widetilde{R}_{i}^{m}$ is upper hemicontinuous (Aliprantis and Border 2006, Theorems 17.23, 17.24). It follows from standard extension theorems (e.g., Lubin 1974) that $\widetilde{R}_{i}^{m}\left(g_{i}^{m}\right)$ is nonempty for any $g_{i}^{m} \in G_{i}^{m}$.

We next relate $R_{i}^{m}$ to $\widetilde{R}_{i}^{m}$ and show that $R_{i}^{m}$ is upper hemicontinuous. Write $\operatorname{proj}_{G_{i}^{m}}$ for the projection function from the set $H_{i}^{\infty} \cup \bigcup_{k \geq m} H_{i}^{k}$ of belief hierarchies of depth at least $m$ into $G_{i}^{m}$. For any $h_{i} \in H_{i}^{\infty} \cup \bigcup_{k \geq m} H_{i}^{k}$, define $\tilde{h}_{i}^{m}:=\operatorname{proj}_{G_{i}^{m}}\left(h_{i}\right)$. Then we can state the following lemma.

Lemma 12. For any $h_{i} \in H_{i}$,

$$
R_{i}^{m}\left(h_{i}\right)= \begin{cases}\widetilde{R}_{i}^{m}\left(\tilde{h}_{i}^{m}\right) & \text { if } h_{i} \in H_{i}^{k} \text { for } k \geq m, \\ \widetilde{R}_{i}^{k}\left(\tilde{h}_{i}^{k}\right) & \text { if } h_{i} \in H_{i}^{k} \text { for } k<m .\end{cases}
$$

${ }^{25}$ Recall that a correspondence $F: X \rightarrow Y$ is upper hemicontinuous if and only if $\{x \in X: F(x) \subset U\}$ for every open subset of $Y$. 
The proof is similar to that of Lemma 7 and thus is omitted. It is now immediate that $R_{i}^{m}$ is nonempty and upper hemicontinuous. Fix $A_{i}^{\prime} \subset A_{i}$. Then

$$
\begin{aligned}
\left\{h_{i} \in H_{i}: R_{i}^{m}\left(h_{i}\right) \subset A_{i}^{\prime}\right\}= & \bigcup_{k=m, m+1, \ldots, \infty}\left\{h_{i} \in H_{i}^{k}: \widetilde{R}_{i}^{m}\left(\tilde{h}_{i}^{m}\right) \subset A_{i}^{\prime}\right\} \\
& \cup \bigcup_{k \leq m-1}\left\{h_{i} \in H_{i}^{k}: \widetilde{R}_{i}^{m}\left(\tilde{h}_{i}^{k}\right) \subset A_{i}^{\prime}\right\} .
\end{aligned}
$$

Each of the sets $\left\{h_{i} \in H_{i}^{k}: \widetilde{R}_{i}^{m}\left(\tilde{h}_{i}^{k}\right) \subset A_{i}^{\prime}\right\}$ is open (Aliprantis and Border 2006, Theorem 17.23). Hence, $\left\{h_{i} \in H_{i}: R_{i}^{m}\left(h_{i}\right) \subset A_{i}^{\prime}\right\}$ is open and $R_{i}^{m}$ is upper hemicontinuous. It then follows that $R_{i}^{\infty}$ is upper hemicontinuous (Aliprantis and Border 2006, Theorem 17.25).

\section{D.3 Proof of Lemma 3}

Since $\psi_{i}$ and the function that maps $\psi_{h_{i}}$ into its marginal on $\Theta$ are both continuous (Proposition 1 and Aliprantis and Border 2006, Theorem 15.14), it suffices to show that the set $\Delta_{i}^{A^{\prime}}$ is nonempty and open in $\Delta(\Theta)$. By assumption, $\Delta_{i}^{A^{\prime}}$ is nonempty. So it remains to show that every element of $\Delta_{i}^{A^{\prime}}$ has a neighborhood in $\Delta_{i}^{A^{\prime}}$.

The first step is to dispose of the quantification in (i) and (ii) in Assumption R$\operatorname{Mult}\left(A^{\prime}\right)$. By Berge's maximum theorem (Aliprantis and Border 2006, Theorem 17.31), for every action $a_{i} \in A_{i}$ for $i$ and for every nonempty subset $B_{-i} \subset A_{-i}$ of actions for the opponent, the correspondence that maps $\theta \in \Theta$ to $\arg \max \left\{u_{i}\left(a_{i}, a_{-i}, \theta\right): a_{-i} \in\right.$ $\left.B_{-i}\right\}$ is upper hemicontinuous. By the Kuratowski-Ryll-Nardzewski selection theorem (Aliprantis and Border 2006, Theorem 18.13), this correspondence admits a measurable selection. Thus, for every $a_{i} \in A_{i}$ and $B_{-i} \subset A_{-i}$, we can fix a measurable function $q_{-i}\left(\cdot \mid a_{i}, B_{-i}\right): \Theta \rightarrow B_{-i}$ such that for all $\theta \in \Theta, u_{i}\left(a_{i}, q_{-i}\left(\theta \mid a_{i}, B_{-i}\right), \theta\right) \geq u_{i}\left(a_{i}, a_{-i}, \theta\right)$ for all $a_{-i} \in B_{-i}$. Hence, $u_{i}\left(a_{i}, q_{-i}\left(\cdot \mid a_{i}, B_{-i}\right), \cdot\right)$ is Borel measurable. We can think of $q_{-i}\left(\cdot \mid a_{i}, B_{-i}\right)$ as the "conjecture" about the opponent's beliefs that gives the highest payoff for $a_{i}$ for every state $\theta \in \Theta$ given that the opponent chooses an action in $B_{-i}$. If $a_{i} \in A_{i}^{\prime}$, the relevant case is the one where the opponent chooses an action in $A_{-i}^{\prime}$; if $a_{i} \notin A_{i}^{\prime}$, we want to allow the opponent to play any action $a_{-i} \in A_{-i}$ (cf. Assumption R$\left.\operatorname{Mult}\left(A^{\prime}\right)\right)$.

We can now rewrite the conditions in Assumption R-Mult $\left(A^{\prime}\right)$ without quantifying over measurable functions $\widetilde{s}_{-i}$. Fix $\mu_{i} \in \Delta_{i}^{A^{\prime}}$. Then the following relationships hold:

(i) For all $a_{i}^{\prime} \in A_{i}^{\prime}$,

$$
\left\{a_{i}^{\prime}\right\}=\underset{\tilde{a}_{i} \in A_{i}}{\arg \max } \int_{\Theta} u_{i}\left(\tilde{a}_{i}, q_{-i}\left(\theta \mid a_{i}^{\prime}, A_{-i}^{\prime}\right), \theta\right) \mathrm{d} \mu_{i}
$$

(ii) For all $a_{i}^{\prime \prime} \notin A_{i}^{\prime}$,

$$
a_{i}^{\prime \prime} \notin \underset{\tilde{a}_{i} \in A_{i}}{\arg \max } \int_{\Theta} u_{i}\left(\tilde{a}_{i}, q_{-i}\left(\theta \mid a_{i}^{\prime \prime}, A_{-i}\right), \theta\right) \mathrm{d} \mu_{i} .
$$


We can now bound the payoff differences. For $a_{i}^{\prime} \in A_{i}^{\prime}$, define

$$
\xi_{a_{i}^{\prime}}:=\min _{a_{i} \neq a_{i}^{\prime}}\left\{\int_{\Theta} u_{i}\left(a_{i}^{\prime}, q_{-i}\left(\theta \mid a_{i}^{\prime}, A_{-i}^{\prime}\right), \theta\right) \mathrm{d} \mu_{i}-\int_{\Theta} u_{i}\left(a_{i}, q_{-i}\left(\theta \mid a_{i}^{\prime}, A_{-i}^{\prime}\right), \theta\right) \mathrm{d} \mu_{i}\right\},
$$

so $\xi_{a_{i}^{\prime}}>0$. For $a_{i}^{\prime \prime} \notin A_{i}^{\prime}$, define

$\zeta_{a_{i}^{\prime \prime}}:=\min _{a_{i} \neq a_{i}^{\prime \prime}}\left\{\max \left\{0, \int_{\Theta} u_{i}\left(a_{i}, q_{-i}\left(\theta \mid a_{i}^{\prime \prime}, A_{-i}\right), \theta\right) \mathrm{d} \mu_{i}-\int_{\Theta} u_{i}\left(a_{i}^{\prime \prime}, q_{-i}\left(\theta \mid a_{i}^{\prime \prime}, A_{-i}\right), \theta\right) \mathrm{d} \mu_{i}\right\}\right\}$.

Again, $\zeta_{a_{i}^{\prime \prime}}>0$. Note that since $u_{i}$ is a continuous function on a compact space, there is $c>0$ such that $u_{i}\left(a_{i}, a_{-i}, \theta\right) \in\left[-\frac{1}{2} c, \frac{1}{2} c\right]$ for all $a_{i}, a_{-i}, \theta$.

The next step is to construct a neighborhood of $\mu_{i}$. We cannot do so directly using the integral of $u_{i}\left(\cdot, q_{-i}\left(\cdot \mid a_{i}, B_{-i}\right), \cdot\right)$ (for $a_{i} \in A_{i}$ and $B_{-i} \subset A_{-i}$ ) because this function may not be continuous if $\Theta$ is uncountably infinite. We can, however, approximate it by a continuous function. By Lusin's theorem (Aliprantis and Border 2006, Theorem 12.8), for each $\eta>0$, there is a compact subset $K_{\eta} \subset \Theta$ such that $\mu_{i}\left(K_{\eta}\right)>1-\eta$ and for all $a_{i}, a_{i}^{\prime} \in A_{i}, B_{-i} \subset A_{-i}$, the restriction of $u_{i}\left(a_{i}, q_{-i}\left(\cdot \mid a_{i}^{\prime}, B_{-i}\right), \cdot\right)$ to $K_{\eta}$, denoted $u_{i}^{\eta}\left(a_{i}, q_{-i}\left(\cdot \mid a_{i}^{\prime}, B_{-i}\right), \cdot\right)$, is continuous. By Tietze's extension theorem (Aliprantis and Border 2006, Theorem 2.47), each function $u_{i}^{\eta}\left(a_{i}, q_{-i}\left(\cdot \mid a_{i}^{\prime}, B_{-i}\right), \cdot\right)$ has a continuous extension $\tilde{u}_{i}^{\eta}\left(a_{i}, q_{-i}\left(\cdot \mid a_{i}^{\prime}, B_{-i}\right), \cdot\right)$ to $\Theta$.

We are now ready to define a neighborhood of $\mu_{i}$ in $\Delta(\Theta)$. For $\tilde{a}_{i} \in A_{i}$, let $\delta^{\tilde{a}_{i}}:=$ $\left(\delta_{a_{i}}^{\tilde{a}_{i}}\right)_{a_{i} \in A_{i}}$ be such that $\delta_{a_{i}}^{\tilde{a}_{i}}>0$ for all $a_{i} \in A_{i}$, and let $\delta:=\left(\delta^{\tilde{a}_{i}}\right)_{\tilde{a}_{i} \in A_{i}}$. For $a_{i}^{\prime} \in A_{i}^{\prime}$, define

$$
\begin{aligned}
O_{i}\left(\mu_{i} ; a_{i}^{\prime}, \eta, \delta\right):= & \bigcap_{a_{i} \in A_{i}}\left\{\nu_{i} \in \Delta(\theta): \mid \int_{\Theta} \tilde{u}_{i}^{\eta}\left(a_{i}, q_{-i}\left(\theta \mid a_{i}^{\prime}, A_{-i}^{\prime}\right), \theta\right) \mathrm{d} \nu_{i}\right. \\
& \left.-\int_{\Theta} \tilde{u}_{i}^{\eta}\left(a_{i}, q_{-i}\left(\theta \mid a_{i}^{\prime}, A_{-i}^{\prime}\right), \theta\right) \mathrm{d} \mu_{i} \mid<\delta_{a_{i}}^{a_{i}^{\prime}}\right\} .
\end{aligned}
$$

Then $O_{i}\left(\mu_{i} ; a_{i}^{\prime}, \eta, \delta\right)$ is open (Billingsley 1968, Appendix III). Moreover, it contains $\mu_{i}$. For $a_{i}^{\prime \prime} \notin A_{i}^{\prime}$, define

$$
\begin{aligned}
O_{i}\left(\mu_{i} ; a_{i}^{\prime \prime}, \eta, \delta\right):= & \bigcap_{a_{i} \in A_{i}}\left\{\nu_{i} \in \Delta(\Theta): \mid \int_{\Theta} \tilde{u}_{i}^{\eta}\left(a_{i}, q_{-i}\left(\theta \mid a_{i}^{\prime \prime}, A_{-i}\right), \theta\right) \mathrm{d} \nu_{i}\right. \\
& \left.-\int_{\Theta} \tilde{u}_{i}^{\eta}\left(a_{i}, q_{-i}\left(\theta \mid a_{i}^{\prime \prime}, A_{-i}\right), \theta\right) \mathrm{d} \mu_{i} \mid<\delta_{a_{i}}^{a_{i}^{\prime \prime}}\right\} .
\end{aligned}
$$

Again, $O_{i}\left(\mu_{i} ; a_{i}^{\prime \prime}, \eta, \delta\right)$ is open and it contains $\mu_{i}$. Define

$$
O_{i}\left(\mu_{i} ; \eta, \delta\right):=\bigcap_{a_{i} \in A_{i}} O_{i}\left(\mu_{i} ; a_{i}, \eta, \delta\right) .
$$


Then $O_{i}\left(\mu_{i} ; \eta, \delta\right)$ is open and it contains $\mu_{i}$. We can then choose $\hat{\eta}>0$ and $\hat{\delta}>0$ such that if $\nu_{i} \in O_{i}\left(\mu_{i} ; \hat{\eta}, \hat{\delta}\right)$, then (i) for all $a_{i}^{\prime} \in A_{i}^{\prime}, a_{i} \neq a_{i}^{\prime}$,

$$
\begin{aligned}
& \int_{\Theta} u_{i}\left(a_{i}^{\prime}, q_{-i}\left(\theta \mid a_{i}^{\prime}, A_{-i}^{\prime}\right), \theta\right) \mathrm{d} \nu_{i}-\int_{\Theta} u_{i}\left(a_{i}, q_{-i}\left(\theta \mid a_{i}^{\prime}, A_{-i}^{\prime}\right), \theta\right) \mathrm{d} \nu_{i} \\
& \geq \xi_{a_{i}^{\prime}}-c \cdot \hat{\eta}-\hat{\delta}_{a_{i}}^{a_{i}^{\prime}}-\hat{\delta}_{a_{i}^{\prime}}^{a_{i}^{\prime}}>0
\end{aligned}
$$

and (ii) for all $a_{i}^{\prime \prime} \notin A_{i}^{\prime}, a_{i} \neq a_{i}^{\prime \prime}$,

$$
\begin{aligned}
& \int_{\Theta} u_{i}\left(a_{i}, q_{-i}\left(\theta \mid a_{i}^{\prime \prime}, A_{-i}\right), \theta\right) \mathrm{d} \nu_{i}-\int_{\Theta} u_{i}\left(a_{i}^{\prime \prime}, q_{-i}\left(\theta \mid a_{i}^{\prime \prime}, A_{-i}\right), \theta\right) \mathrm{d} \nu_{i} \\
& \geq \zeta_{a_{i}^{\prime \prime}}-c \cdot \hat{\eta}-\hat{\delta}_{a_{i}}^{a_{i}^{\prime \prime}}-\hat{\delta}_{a_{i}^{a_{i}^{\prime \prime}}}^{a^{\prime \prime}}>0 .
\end{aligned}
$$

Then $O_{i}\left(\mu_{i} ; \hat{\eta}, \hat{\delta}\right) \subset \Delta_{i}^{A^{\prime}}$. It follows that $\Delta_{i}^{A^{\prime}}$ is open.

\section{D.4 Proof of Lemma 4}

Let $m=0,1,2, \ldots, \infty$. Suppose that $V_{-i} \subset H_{-i}$ is open and that for all $h_{-i} \in V_{-i}$, $R_{-i}^{m}\left(h_{-i}\right)=A_{-i}^{\prime}$. Let $h_{i} \in H_{i}$ be a type with $\operatorname{marg}_{\Theta} \psi_{h_{i}} \in \Delta_{i}^{A^{\prime}}$ and $\psi_{h_{i}}\left(V_{-i}\right)=1$. Then, clearly, $R_{i}^{m+1}\left(h_{i}\right)=A_{i}^{\prime}: h_{i}$ assigns probability 1 to types that can play precisely the actions in $A_{-i}^{\prime}$, and the actions that are a best response are precisely the actions in $A_{i}^{\prime}$. It remains to show that $h_{i}$ has a neighborhood such that $R_{i}^{m+1}\left(h_{i}^{\prime}\right)=A_{i}^{\prime}$ for every type $h_{i}^{\prime}$ in the neighborhood. It is convenient to define

$$
D_{i}\left(a_{i}, a_{i}^{\prime}, a_{-i}, \theta\right):=u_{i}\left(a_{i}, a_{-i}, \theta\right)-u_{i}\left(a_{i}^{\prime}, a_{-i}, \theta\right)
$$

for $a_{i}, a_{i}^{\prime} \in A_{i}, a_{-i} \in A_{-i}$, and $\theta \in \Theta$. The definition can be extended to mixed strategies in the obvious way. Since the $(m$-) rationalizability correspondence is upper hemicontinuous (Lemma 2), it follows from the Kuratowski-Ryll-Nardzewski selection theorem (Aliprantis and Border 2006, Theorem 18.13) that for each $a_{i}^{\prime} \in A_{i}^{\prime}$, there is a (measurable) conjecture $s_{-i}^{a_{i}^{\prime}}: \Theta \times H_{-i} \rightarrow \Delta\left(A_{-i}\right)$ such that

$$
\begin{aligned}
& \operatorname{supp} s_{-i}^{a_{i}^{\prime}}\left(\theta, h_{-i}\right) \subset R_{-i}^{m-1}\left(h_{-i}\right) \quad \text { for all } \theta, h_{-i}, \\
& \int D_{i}\left(a_{i}^{\prime}, a_{i}, s_{-i}^{a_{i}^{\prime}}(\theta, h), \theta\right) \mathrm{d} \psi_{h_{i}}>0 \text { for all } a_{i} \neq a_{i}^{\prime}, \\
& s_{-i}^{a_{i}^{\prime}}\left(\theta, h_{-i}\right)=q_{-i}\left(\theta \mid a_{i}^{\prime}, A_{-i}^{\prime}\right) \text { for } h_{-i} \in V_{-i}^{m-1},
\end{aligned}
$$

where $q_{-i}\left(\theta \mid a_{i}^{\prime}, A_{-i}^{\prime}\right)$ was defined in the proof of Lemma 3. Likewise, for $a_{i}^{\prime \prime} \notin A_{i}^{\prime}$, for every measurable conjecture $s_{-i}^{a_{i}^{\prime \prime}}: \Theta \times H_{-i} \rightarrow \Delta\left(A_{-i}\right)$ such that $\operatorname{supp} s_{-i}^{a_{i}^{\prime \prime}}\left(\theta, h_{-i}\right) \subset$ $R_{-i}^{m-1}\left(h_{-i}\right)$, there is $a_{i} \neq a_{i}^{\prime \prime}$ such that

$$
\int D_{i}\left(a_{i}^{\prime \prime}, a_{i}, s_{-i}^{a_{i}^{\prime \prime}}(\theta, h), \theta\right) \mathrm{d} \psi_{h_{i}}<0 .
$$


For $v>0$, define

$$
O_{i}\left(h_{i} ; v\right):=\left\{h_{i}^{\prime} \in H_{i}: \psi_{h_{i}^{\prime}}\left(V_{-i}\right)>\psi_{h_{i}}\left(V_{-i}\right)-v\right\} .
$$

This set is open (Billingsley 1968, Appendix III) and it contains $h_{i}$. For $\eta>0$ and $\delta=$ $\left(\delta a_{a_{i}}\right)_{a_{i}, \tilde{a}_{i} \in A_{i}}$ with $\delta_{a_{i}}^{\tilde{a}_{i}}>0$ for $a_{i}, \tilde{a}_{i}$, define

$$
O_{i}\left(h_{i} ; \eta, \delta\right):=\left\{h_{i}^{\prime} \in H_{i}: \operatorname{marg}_{\Theta} \psi_{h_{i}^{\prime}} \in O_{i}\left(\operatorname{marg}_{\Theta} \psi_{h_{i}} ; \eta, \delta\right)\right\},
$$

where we have again used the notation from Lemma 3. Again, this set is open and contains $h_{i}$. Consequently, the set

$$
O_{i}\left(h_{i} ; \eta, \delta, v\right):=O_{i}\left(h_{i} ; v\right) \cap O_{i}\left(h_{i} ; \eta, \delta\right)
$$

is nonempty and open. By a similar argument as in the proof of Lemma 3, if we choose $\hat{\eta}, \hat{v}, \hat{\delta}>0$ sufficiently close to 0 , the actions in $A_{i}^{\prime}$ are (strictly) $m$-rationalizable for the types in $O_{i}\left(h_{i} ; \hat{\eta}, \hat{\delta}, \hat{v}\right)$, and no other actions are $m$-rationalizable for these types (i.e., $R_{i}^{m}\left(h_{i}^{\prime}\right)=A_{i}^{\prime}$ for $\left.h_{i}^{\prime} \in O_{i}\left(h_{i} ; \hat{\eta}, \hat{\delta}, \hat{v}\right)\right)$. Let $O\left(h_{i}\right):=O_{i}\left(h_{i} ; \hat{\eta}, \hat{\delta}, \hat{v}\right)$.

\section{D.5 Proof of Lemma 5}

By Lemma 3, the set $S_{i}^{1}:=\left\{h_{i} \in H_{i}: R_{i}^{1}\left(h_{i}\right)=A_{i}^{\prime}\right\}$ is nonempty and open. In particular, the interior $U_{i}^{1}:=S_{i}^{1}$ of $S_{i}^{1}$ is nonempty. It follows that $A_{i}^{\prime}$ is robustly 1-rationalizable for the types in $U_{i}^{1}$. Since $\mathcal{T}^{*}$ is complete (Appendix B), there is a type $h_{i} \in U_{i}^{1}$ that assigns probability 1 to $U_{-i}^{1}$.

For $m>1$, suppose that the set $S_{i}^{m-1}:=\left\{h_{i} \in H_{i}: R_{i}^{m-1}\left(h_{i}\right)=A_{i}^{\prime}\right\}$ has a nonempty open subset $U_{i}^{m-1}$ and that there is a type $h_{i} \in U_{i}^{m-1}$ that assigns probability 1 to $U_{-i}^{m-1}$. By construction, $h_{i}$ has a belief in the multiplicity set (i.e., $h_{i} \in \Delta_{i}^{A^{\prime}}$ ). By Lemma $4, h_{i}$ has a neighborhood $O\left(h_{i}\right)$ such that $R_{i}^{m}\left(h_{i}^{\prime}\right)=A_{i}^{\prime}$ for $h_{i}^{\prime} \in O\left(h_{i}\right)$. Consequently, $A_{i}^{\prime}$ is robustly $m$-rationalizable for $h_{i}$. Let $U_{i}^{m}$ be the union of such neighborhoods $O\left(h_{i}\right)$ (where $h_{i}$ ranges over the types in $U_{i}^{m-1}$ that assign probability 1 to $U_{-i}^{m-1}$ ). So $U_{i}^{m}$ is a nonempty open subset of $S_{i}^{m}:=\left\{h_{i} \in H_{i}: R_{i}^{\infty}\left(h_{i}\right)=A_{i}^{\prime}\right\}$. Again, by completeness, there exist types in $U_{i}^{m}$ that assign probability 1 to $U_{-i}^{m}$.

\section{D.6 Proof of Proposition 4 (cont.)}

Consider a type $h_{i}^{0} \in B_{i}^{0}$ with beliefs in the multiplicity set $\Delta_{i}^{A^{\prime}}$ that assigns probability 1 to $\bigcup_{m} V_{-i}^{m}$. (Such a type exists since $\mathcal{T}^{*}$ is complete; see Appendix B.) By Lemma 4, $h_{i}^{0}$ has a neighborhood $O\left(h_{i}^{0}\right)$ such that $R_{i}^{\infty}\left(h_{i}^{\prime}\right)=A_{i}^{\prime}$ for $h_{i}^{\prime} \in O\left(h_{i}^{0}\right)$. It follows that $A_{i}^{\prime}$ is robustly rationalizable for $h_{i}^{0}$. For $n>0$, suppose that there is a type $h_{-i}^{n-1} \in B_{-i}^{n-1}$ such that $A_{-i}^{\prime}$ is robustly rationalizable for $h_{-i}^{n-1}$. That is, $h_{-i}^{n-1}$ has a neighborhood $O\left(h_{-i}^{n-1}\right)$ such that $R_{-i}^{\infty}\left(h_{-i}\right)=A_{-i}^{\prime}$. Consider a type $h_{i}^{n} \in B_{i}^{n}$ that assigns probability 1 to $O\left(h_{-i}^{n-1}\right)$. Then, by Lemma $4, A_{i}^{\prime}$ is robustly rationalizable for $h_{i}$. 


\section{D.7 Proof of Lemma 6}

We use the following auxiliary result.

CLAim 1. There exist $\underline{x}_{1}^{\varepsilon}, \underline{x}_{2}^{\varepsilon}, \ldots$ and $\underline{x}_{1}^{\varepsilon}, \underline{x}_{2}^{\varepsilon}, \ldots$ such that for every $k, 0 \leq \underline{x}_{k}^{\varepsilon}<\frac{1}{2}<\bar{x}_{k}^{\varepsilon} \leq 1$ and for every depth-k type $h_{i}$ in $T^{\varepsilon}$ with signal $x_{i} \in X_{i}^{\varepsilon}$,

- not investing is the unique rationalizable action whenever $x_{i}<\underline{x}_{k}^{\varepsilon}$,

- investing is the unique rationalizable actions whenever $x_{i}>\bar{x}_{k}^{\varepsilon}$,

- both actions are rationalizable whenever $x_{i} \in\left[\underline{x}_{k}^{\varepsilon}, \bar{x}_{k}^{\varepsilon}\right]$.

Moreover, for every $k, \underline{x}_{k}^{\varepsilon} \rightarrow 0$ and $\bar{x}_{k}^{\varepsilon} \rightarrow 1$ as $\varepsilon \rightarrow 0$.

Proof. Consider a depth-1 type $h_{i}$ in $T^{\varepsilon}$. Then $N I$ is the unique rationalizable action for $h_{i}$ if and only if its signal $x_{i}$ is less than 0 , and $I$ is its unique rationalizable action if and only if $x_{i}>1$. Let $x_{1}^{\varepsilon}:=0$ and $\bar{x}_{1}^{\varepsilon}:=1$. For $k>1$, suppose the claim is true for $k-1$. If the game has complete information (i.e., $\varepsilon=0$ ), then we can set $\underline{x}_{k}=\underline{x}_{k-1}$ and $\bar{x}_{k}=\bar{x}_{k-1}$, and we are done. So suppose $\varepsilon>0$. Consider a type in $T^{\varepsilon}$ with signal $x_{i} \in[-1+\varepsilon, 2-\varepsilon]$, and fix $z \in\left[x_{i}-\varepsilon, x_{i}\right]$. The posterior probability that the type assigns to the opponent having signal $x_{-i} \leq z$ is then

$$
\pi^{\varepsilon}\left(z ; x_{i}\right)=\int_{x_{i}-\varepsilon}^{z+\varepsilon}\left(\int_{\theta-\varepsilon}^{z} \frac{\mathrm{d} x_{-i}}{2 \varepsilon}\right) \frac{\mathrm{d} \theta}{2 \varepsilon}=\frac{1}{8 \varepsilon^{2}}\left(z-x_{i}+2 \varepsilon\right)^{2} .
$$

If the type has depth $k$, its expected payoff to $I$ is at most

$$
\left(1-\pi^{\varepsilon}\left(\underline{x}_{k-1}^{\varepsilon} ; x_{i}\right)\right) \cdot x_{i}+\pi^{\varepsilon}\left(\underline{x}_{k-1}^{\varepsilon} ; x_{i}\right) \cdot\left(x_{i}-1\right) \text {, }
$$

and it is at least

$$
\left(1-\pi^{\varepsilon}\left(\bar{x}_{k-1}^{\varepsilon} ; x_{i}\right)\right) \cdot x_{i}+\pi^{\varepsilon}\left(\bar{x}_{k-1}^{\varepsilon} ; x_{i}\right) \cdot\left(x_{i}-1\right) .
$$

Consequently, not investing is the unique rationalizable action for the type if $x_{i}<\underline{x}_{k}^{\varepsilon}$ and investing is the unique rationalizable action if $x_{i}>\bar{x}_{k}^{\varepsilon}$, where $x_{k}^{\varepsilon}=\underline{x}_{k}^{\varepsilon}, \bar{x}_{k}^{\varepsilon}$ solves $x_{k}^{\varepsilon}=$ $\pi^{\varepsilon}\left(x_{k-1}^{\varepsilon} ; x_{k}^{\varepsilon}\right)$, that is,

$$
x_{k}^{\varepsilon}=4 \varepsilon^{2}+2 \varepsilon+x_{k-1}^{\varepsilon}-4 \varepsilon \sqrt{\varepsilon^{2}+\frac{1}{2} x_{k-1}^{\varepsilon}+\varepsilon} .
$$

The function $f^{\varepsilon}(z)=4 \varepsilon^{2}+2 \varepsilon+z-4 \varepsilon \sqrt{\varepsilon^{2}+\frac{1}{2} z+\varepsilon}$ is increasing in $z$, with $f^{\varepsilon}(0)>0$ and $f^{\varepsilon}(1)<1$. Finally, the equation $f^{\varepsilon}(z)=z$ has a unique solution at $z=\frac{1}{2}$. This proves the first part of the claim.

We next show that the thresholds $\underline{x}_{k}^{\varepsilon}$ and $\bar{x}_{k}^{\varepsilon}$ converge to 0 and 1 , respectively, as $\varepsilon \rightarrow 0$. To show this, note that $\lim _{\varepsilon \rightarrow 0} f^{\varepsilon}\left(\underline{x}_{1}^{\varepsilon}\right)=0$ and $\lim _{\varepsilon \rightarrow 0} f^{\varepsilon}\left(\bar{x}_{1}^{\varepsilon}\right)=1$. For $k>1$, suppose, inductively, that $\lim _{\varepsilon \rightarrow 0} f^{\varepsilon}\left(\underline{x}_{k-1}^{\varepsilon}\right)=0$ and $\lim _{\varepsilon \rightarrow 0} f^{\varepsilon}\left(\bar{x}_{k-1}^{\varepsilon}\right)=1$. Then it follows directly that $\lim _{\varepsilon \rightarrow 0} f^{\varepsilon}\left(\underline{x}_{k}^{\varepsilon}\right)=0$ and $\lim _{\varepsilon \rightarrow 0} f^{\varepsilon}\left(\bar{x}_{k}^{\varepsilon}\right)=1$. 
By Claim 1, both actions are strictly rationalizable for a depth- $k$ type in $T^{\varepsilon}$ whenever its signal $x_{i}$ lies strictly between $\underline{x}_{k}^{\varepsilon}$ and $\bar{x}_{k}^{\varepsilon}$. It remains to show that multiplicity is robust. Consider a depth-1 type $h_{i}$ in $T^{\varepsilon}$ with $x_{i} \in\left(\underline{x}_{1}^{\varepsilon}, \bar{x}_{1}^{\varepsilon}\right)$. For $\delta>0$, define

$$
O_{i}\left(x_{i}, 1 ; \delta\right):=\left\{h_{i} \in H_{i}^{1}: \int \theta \mathrm{d} \psi_{h_{i}} \in\left(x_{i}-\delta, x_{i}+\delta\right)\right\} .
$$

Then $O_{i}\left(x_{i}, 1 ; \delta\right)$ contains $h_{i}$ and is open in $H_{i}$ (Billingsley 1968, Appendix III). If we choose $\delta^{*}>0$ sufficiently small, then both actions are strictly rationalizable for the types in $O_{i}\left(x_{i}, 1 ; \delta^{*}\right)$. Define $O_{x_{i}, 1}:=O_{i}\left(x_{i}, 1 ; \delta^{*}\right)$. It follows that multiplicity is robust for $h_{i}$.

For $k>1$, the proof is a little more involved because depth- $k$ types have nontrivial beliefs about the rationalizable strategies of the opponent. Fix a depth- $k$ type $h_{i}$ in $T^{\varepsilon}$ with signal $x_{i} \in\left(\underline{x}_{k}^{\varepsilon}, \bar{x}_{k}^{\varepsilon}\right)$ and write $p_{x_{i}, k}$ for the probability that $h_{i}$ assigns to the opponent having a signal in $\left(\underline{x}_{k-1}^{\varepsilon}, \bar{x}_{k-1}^{\varepsilon}\right)$. Since both actions are strictly rationalizable for $h_{i}$, we have $p_{x_{i}, k}>x_{i}, 1-x_{i}$. Since $\psi_{h_{i}}$ is regular, for every $\eta>0$, there is a compact subset $K_{\eta}$ of the set of types in $T_{-i}^{\varepsilon, k-1}$ with signal $x_{-i} \in\left(\underline{x}_{k-1}^{\varepsilon}, \bar{x}_{k-1}^{\varepsilon}\right)$ such that $\psi_{h_{i}}\left(K_{\eta}\right)>$ $p_{x_{i}, k}-\eta$. Since $K_{\eta}$ is compact, it has a (finite) open cover $V_{x_{i}, k, \eta}:=\bigcup_{m=1}^{\ell} O_{x_{i}^{m}, k-1}$, where $x_{i}^{m} \in\left(\underline{x}_{k-1}^{\varepsilon}, \bar{x}_{k-1}^{\varepsilon}\right)$. Therefore, $\psi_{h_{i}}\left(V_{x_{i}, k, \eta}\right)>p_{x_{i}, k}-\eta$. For $\xi, \delta>0$, define

$$
\begin{aligned}
O_{i}\left(x_{i}, k ; \eta, \xi, \delta\right):= & \left\{h_{i}^{\prime} \in H_{i}: \psi_{h_{i}^{\prime}}\left(V_{x_{i}, k, \eta}\right)>p_{x_{i}, k}-\eta-\xi\right\} \\
& \cap\left\{h_{i}^{\prime} \in H_{i}: \int \theta \mathrm{d} \psi_{h_{i}^{\prime}} \in\left(x_{i}-\delta, x_{i}+\delta\right)\right\} .
\end{aligned}
$$

Then $O_{i}\left(x_{i}, k ; \xi, \delta\right)$ clearly contains $h_{i}$; moreover, it is open (Billingsley 1968, Appendix III). By choosing $\eta^{*}, \xi^{*}, \delta^{*}>0$ sufficiently close to 0 , we can ensure that both actions are strictly rationalizable for the types in $O_{i}\left(x_{i}, k ; \eta^{*}, \xi^{*}, \delta^{*}\right)$. Let $O_{x_{i}, k}:=$ $O_{i}\left(x_{i}, k ; \eta^{*}, \xi^{*}, \delta^{*}\right)$. Again, multiplicity is robust for any $\varepsilon \in\left[0, \frac{1}{2}\right)$.

\section{D.8 Proof of Proposition 5}

Each type in $T^{\varepsilon}$ is characterized completely by its signal $x_{i}$ and its reasoning ability (i.e., its depth of reasoning and higher-order beliefs about players' depth of reasoning). We can quantify the latter by assigning a rank to each type. For $n<\infty$, the rank of a type $h_{i} \in T_{i}^{\varepsilon, n}$ is just its depth $n$. The rank of the other types can be assigned using transfinite ordinals. Define the rank of a type $h_{i}$ in $T_{i}^{\varepsilon, \infty}$ to be $\omega$ (where $\omega$ is the first countable ordinal), and for $n>0$, let the rank of a type in $T^{\varepsilon, \infty+n}$ be $\omega+n$. Types with the same rank have the same depth of reasoning and the same higher-order beliefs about depth of reasoning.

By Lemma 6, for every finite $\alpha$ and any $\varepsilon \in\left[0, \frac{1}{2}\right)$, there is $\underline{x}_{\alpha}^{\varepsilon} \in\left(0, \frac{1}{2}\right)$ and $\bar{x}_{\alpha}^{\varepsilon} \in\left(\frac{1}{2}, 1\right)$ such that for every type $h_{i}$ in $T^{\varepsilon}$ with rank $\alpha$ and signal $x_{i} \in\left(\underline{x}_{\alpha}^{\varepsilon}, \bar{x}_{\alpha}^{\varepsilon}\right)$, both actions are robustly rationalizable for $h_{i}$.

Let $\alpha=\omega$ and fix $z \in\left(\frac{1}{2}, 1\right)$. Then there is finite $k_{z}$ such that any type in $T^{\varepsilon}$ with rank $\alpha$ assigns probability less than $z$ to types with rank greater than $k_{z}$. By construction, $k_{z}$ does not depend on $\varepsilon$.

For ease of notation, write $\underline{x}:=\underline{x}_{k_{z}}^{\varepsilon}$ and $\bar{x}:=\bar{x}_{k_{z}}^{\varepsilon}$. Fix $\tilde{x}_{i} \in\left(0, \frac{1}{2}\right)$ such that $\tilde{x}_{i}>1-z, \underline{x}$ and hence $\tilde{x}_{i}<z, \bar{x}$ (as $z, \bar{x}>\frac{1}{2}$ ). (Such a signal $\tilde{x}_{i}$ exists, since $1-z, \underline{x}<\frac{1}{2}$.) 
Then there is $\varepsilon_{\tilde{x}_{i}}>0$ such that if $\varepsilon \leq \varepsilon_{\tilde{x}_{i}}$, a type in $T^{\varepsilon}$ with rank $\alpha$ and signal $y_{i} \in\left[\tilde{x}_{i}, \frac{1}{2}\right]$ assigns probability 1 to the opponent having a signal in $(\underline{x}, \bar{x})$. If the type conjectures that the opponent invests whenever his rank is $k \leq k_{z}$ and his signal is in $\left(\underline{x}_{k}^{\varepsilon}, \bar{x}_{k}^{\varepsilon}\right) \supset$ $(\underline{x}, \bar{x})$, then the expected payoff to $I$ is at least $(1-z) \cdot y_{i}+z \cdot\left(y_{i}-1\right)>0$. Under the conjecture that the opponent does not invest whenever his rank is $k \leq k_{z}$ and his signal is in $\left(\underline{x}_{k}^{\varepsilon}, \bar{x}_{k}^{\varepsilon}\right) \supset(\underline{x}, \bar{x})$, the expected payoff to $I$ is less than $z \cdot \frac{1}{2}-(1-z) \cdot \frac{1}{2}=0$. Thus, if $\varepsilon<\varepsilon_{x_{i}}$, then both actions are strictly rationalizable for a type in $T^{\varepsilon}$ with rank $\alpha$ and signal $y_{i} \in\left[\tilde{x}_{i}, \frac{1}{2}\right]$. Likewise, for $\tilde{x}_{i}^{\prime} \in\left(\frac{1}{2}, 1\right)$, there is $\varepsilon_{\tilde{x}_{i}^{\prime}}>0$ such that both actions are strictly rationalizable for a type in $T^{\varepsilon}$ with rank $\alpha$ and signal $y_{i} \in\left[\frac{1}{2}, \tilde{x}_{i}^{\prime}\right]$. Define $\underline{x}_{\alpha}:=\tilde{x}_{i}$, $\bar{x}_{\alpha}:=\tilde{x}_{i}^{\prime}$, and $\tilde{\varepsilon}:=\min \left\{\varepsilon_{\tilde{x}_{i}}, \varepsilon_{\tilde{x}_{i}^{\prime}}\right\}$.

For $n>0$, suppose that for $\gamma=\omega, \omega+1, \ldots, \omega+n-1$, there exist $\varepsilon_{\gamma}>0, \underline{x}_{\gamma}^{\varepsilon}<\frac{1}{2}$, and $\bar{x}_{\gamma}^{\varepsilon}>\frac{1}{2}$ such that for any rank- $\gamma$ type in $T^{\varepsilon}$, both actions are strictly rationalizable whenever its signal lies strictly between $\underline{x}_{\gamma}^{\varepsilon}$ and $\bar{x}_{\gamma}^{\varepsilon}$, and $\varepsilon<\varepsilon_{\gamma}$. Fix $\tilde{x}_{i} \in\left(x_{\omega+n-1}^{\varepsilon}, \frac{1}{2}\right)$. Then there is $\varepsilon_{\tilde{x}_{i}}>0$ such that if $\varepsilon<\varepsilon_{\tilde{x}_{i}}$, a type in $T^{\varepsilon}$ assigns probability 1 to the opponent having a type in $\left(\underline{x}_{\omega+n-1}^{\varepsilon}, \bar{x}_{\omega+n-1}^{\varepsilon}\right)$. Hence, if $\varepsilon<\min \left\{\varepsilon_{\omega}, \ldots, \omega_{\omega+n-1}, \varepsilon_{\tilde{x}_{i}}\right\}$, both actions are strictly rationalizable for a rank- $(\omega+n)$ type in $T^{\varepsilon}$ with signal $\tilde{x}_{i}$. By a similar argument, for a fixed $\tilde{x}_{i}^{\prime} \in\left(\frac{1}{2}, x_{\omega+n-1}^{\varepsilon}\right)$, we can find $\varepsilon_{\tilde{x}_{i}^{\prime}}>0$ such that both actions are strictly rationalizable for any rank- $(\omega+n)$ type in $T^{\varepsilon}$ with signal $\tilde{x}_{i}^{\prime}$ whenever $\varepsilon<\min \left\{\varepsilon_{\omega}, \ldots, \omega_{\omega+n-1}, \varepsilon_{\tilde{x}_{i}^{\prime}}\right\}$. Define $\underline{x}_{\alpha}^{\varepsilon}:=\tilde{x}_{i}, \bar{x}_{\alpha}^{\varepsilon}:=\tilde{x}_{i}^{\prime}$, and $\varepsilon_{\omega+n}:=$ $\min \left\{\varepsilon_{\omega}, \ldots, \omega_{\omega+n-1}, \varepsilon_{\tilde{x}_{i}}, \varepsilon_{\tilde{x}_{i}^{\prime}}\right\}$.

Accordingly, for every infinite rank $\alpha$, there exist $\varepsilon_{\alpha}>0$ and bounds $\underline{x}_{\alpha}^{\varepsilon}<\frac{1}{2}$ and $\bar{x}_{\alpha}^{\varepsilon}>\frac{1}{2}$ such that both actions are strictly rationalizable for a rank- $\alpha$ type in $T^{\varepsilon}$ with a signal strictly between these bounds whenever $\varepsilon<\varepsilon_{\alpha}$. We next show that this multiplicity is robust. Recall that by Lemma 6, for every finite rank $\gamma$, there exist $\underline{x}_{\gamma}^{\varepsilon} \in\left(0, \frac{1}{2}\right)$ and $\bar{x}_{\gamma}^{\varepsilon} \in\left(\frac{1}{2}, 1\right)$ such that every rank- $\gamma$ type in $T^{\varepsilon}$ with signal $x_{i} \in\left(\underline{x}_{\gamma}^{\varepsilon}, \bar{x}_{\gamma}^{\varepsilon}\right)$ has a neighborhood $O_{x_{i}, \gamma}$ such that both actions are rationalizable for the types in $O_{x_{i}, \gamma}$.

Suppose $\varepsilon<\varepsilon_{\omega}$, and fix a type $h_{i}$ in $T^{\varepsilon}$ with rank $\omega$ and signal $x_{i} \in\left(\underline{x}_{\omega}^{\varepsilon}, \bar{x}_{\omega}^{\varepsilon}\right)$. By construction, $h_{i}$ assigns probability 1 to the opponent having a signal in $\left(\underline{x}_{k_{z}}^{\varepsilon}, \bar{x}_{k_{z}}^{\varepsilon}\right)$ and probability less than $z$ to types with rank greater than $k_{z}$. Since $\psi_{h_{i}}$ is a regular probability measure, for every $\eta>0$, there is a compact subset $K_{\eta}$ of types that have rank at most $k_{z}$ and whose signal is in $\left(\underline{x}_{k_{z}}^{\varepsilon}, \bar{x}_{k_{z}}^{\varepsilon}\right)$ such that $\psi_{h_{i}}\left(K_{\eta}\right)>1-z-\eta$. Moreover, $K_{\eta}$ has a (finite) open cover $V_{x_{i}, \omega, \eta}:=\bigcup_{m=1}^{\ell} O_{x_{i}^{m}, \gamma^{m}}$, where $\gamma^{m} \leq k_{z}$ and $x_{i}^{m} \in\left(\underline{x}_{\gamma^{m}}^{\varepsilon}, \bar{x}_{\gamma^{m}}^{\varepsilon}\right)$. Then, by a similar argument as before, we can construct a neighborhood $O_{x_{i}, \omega}$ of $h_{i}$ such that both actions are strictly rationalizable across all types in the neighborhood. The proof for $\alpha=\omega+1, \omega+2, \ldots$ is now straightforward and thus is omitted.

\section{D.9 Proof of Proposition 6}

Let $\hat{H}_{i}$ be the set of types from finite models. ${ }^{26}$ Fix a finite model $M=(\widetilde{\Theta}, T)$ consistent with common belief in an infinite depth, and let $h_{i}^{*} \in T_{i}$ be a type with multiple rationalizable actions. That is, $R_{i}^{\infty}\left(h_{i}^{*}\right) \supset\{a, b\}$ for two distinct actions $a, b \in A_{i}$.

${ }^{26}$ That is, $h_{i} \in \hat{H}_{i}$ if and only if there is a finite model $M^{\prime \prime}=\left(\widetilde{\Theta}^{\prime \prime}, T^{\prime \prime}\right)$ such that $h_{i} \in T_{i}^{\prime \prime}$. 
Fix $\eta>0$ and $\kappa<\infty$. By Corollary 2 of Weinstein and Yildiz (2007), every $(\eta, \kappa)$-ball $O_{\eta, \kappa}\left(h_{i}^{*}\right)$ of $h_{i}^{*}$ has a nonempty intersection with the sets $U_{i}^{a}:=\left\{h_{i} \in \hat{H}_{i}: R_{i}^{\infty}\left(h_{i}\right)=\{a\}\right\}$ and $U_{i}^{b}:=\left\{h_{i} \in \hat{H}_{i}: R_{i}^{\infty}\left(h_{i}\right)=\{b\}\right\}$ of types for whom $a$ and $b$, respectively, are the unique rationalizable action. Let $h_{i}^{a} \in U_{i}^{a} \cap O_{\varepsilon}\left(h_{i}^{*}\right)$ and $h_{i}^{b} \in U_{i}^{b} \cap O_{\varepsilon}\left(h_{i}^{*}\right)$, so $R_{i}^{\infty}\left(h_{i}^{a}\right)=\{a\}$ and $R_{i}^{\infty}\left(h_{i}^{b}\right)=\{b\}$.

Define the model $\left(M^{e}, \tau^{e}\right), e=a, b$, as follows. For $e=a, b$, let $\tilde{M}^{e}=\left(\widetilde{\Theta}^{e}, \tilde{T}^{e}\right)$ be a finite model that contains $h_{i}^{e}$. (Such a model exists, since $h_{i}^{e} \in \hat{H}_{i}$.) Then let $M^{e}=$ $\left(\widetilde{\Theta} \cup \widetilde{\Theta}^{e}, T^{e}\right)$, where $T_{i}^{e}:=T_{i} \cup \tilde{T}_{i}^{e}$ for $i=1,2$. Define $\tau^{e}: T \rightarrow T^{e}$ by $\tau_{i}^{e}\left(h_{i}^{*}\right):=h_{i}^{e}$ and define $\tau_{j}^{e}\left(h_{j}\right)=h_{j}$ for $h_{j} \neq h_{i}^{*}$. Then $\left(M^{a}, \tau^{a}\right)$ and $\left(M^{b}, \tau^{b}\right)$ are $(\eta, \kappa)$ perturbations of $M$.

If an equilibrium $\sigma$ is $(\eta, \kappa)$-robust, then there is a Bayesian-Nash equilibrium $\sigma^{a}$ for $M^{a}$ and a Bayesian-Nash equilibrium $\sigma^{b}$ for $M^{b}$ such that

$$
\sigma_{i}^{a}\left(h_{i}^{a}\right)=\sigma_{i}^{a}\left(\tau_{i}^{a}\left(h_{i}^{*}\right)\right)=\sigma_{i}\left(h_{i}^{*}\right)=\sigma_{i}^{b}\left(\tau_{i}^{b}\left(h_{i}^{*}\right)\right)=\sigma_{i}^{b}\left(h_{i}^{b}\right) .
$$

But, as equilibrium refines rationalizability, $\sigma_{i}^{a}\left(h_{i}^{a}\right)=a$ and $\sigma_{i}^{b}\left(h_{i}^{b}\right)$. So $\sigma$ is not $(\eta, \kappa)$ robust. Since a similar argument holds for any Bayesian-Nash equilibrium and $\eta>0$, $\kappa<\infty$, there is no robust equilibrium for $M$.

\section{D.10 Proof of Proposition 7}

As noted in the main text, we take $X_{i}$ to be a singleton here. It should be clear how to extend the result to the general case.

We construct a set of models, one model $M^{A^{\prime}}$ with beliefs in the multiplicity set $\Delta^{A^{\prime}}$ and one model $M^{a}$ for each action profile $a^{\prime}$ (possibly empty). We then use these models to define a larger model $M$. Considering the models $M^{a}$ is not necessary to prove the result. However, it is instructive to compare the robustness proof for the types with multiplicity (in $M^{A^{\prime}}$ ) with the proof for the types with a dominant action (in $M^{a}$ ).

Step 1. Defining the model $M^{A^{\prime}}$. Fix $k^{A^{\prime},<\infty}=0,1, \ldots$ and $k^{A^{\prime}, \infty}=1,2, \ldots$ Let $T_{i}^{A^{\prime}, 0}:=H_{i}^{0}$ and, for $m=1, \ldots, k^{A^{\prime},<\infty}$, let $T_{i}^{A^{\prime}, m}$ be a finite set of depth- $m$ types with belief in $\Delta_{i}^{A^{\prime}}$ that assign probability 1 to $T_{-i}^{A^{\prime}, m-1}$. For $m=1, \ldots, k^{A^{\prime}, \infty}$, let $T_{i}^{A^{\prime}, k^{<\infty}+m}$ be a finite set of infinite-depth types with belief in $\Delta_{i}^{A^{\prime}}$ that assign probability 1 to $\bigcup_{\ell=1}^{k^{A^{\prime},<\infty}+m-1} T_{-i}^{A^{\prime}, \ell}$.

Let $T_{i}^{A^{\prime}}:=\bigcup_{\ell=0}^{k^{A^{\prime},<\infty}+k^{A, \infty}} T_{i}^{A^{\prime}, \ell}$. Then $M^{A^{\prime}}:=\left(\widetilde{\Theta}^{A^{\prime}}, T^{A^{\prime}}\right)$ is a finite model. Every type in $T_{i}^{A^{\prime}, m}$ assigns probability 1 to types in $\bigcup_{\ell<m} T_{-i}^{A^{\prime}, \ell}$. Say that the type rank of a type $t_{i} \in T_{i}^{A^{\prime}, m}$ is $m$. By construction, each type in $M^{A^{\prime}}$ has a unique type rank. The model $M^{A^{\prime}}$ has level- $k$ beliefs; however, this is immaterial for our results.

Step 2. Defining the model $M^{a}$ for $a \in A$. Fix an action profile $a \in A$. If there is a player $i$ for whom there is no state at which $a_{i}$ is strictly dominant, then we define $T^{a}=\varnothing, \widetilde{\Theta}^{a}=$ $\varnothing$. (Of course, this is ruled out if Assumption R-Dom is satisfied.) Otherwise, for $i=1,2$, let $\theta^{a_{i}}$ be a state for which $a_{i}$ is strictly dominant for $i$. Fix $k^{a,<\infty}=0,1, \ldots$ and $k^{a, \infty}=$ $1,2, \ldots$ Let $T_{i}^{a, 0}:=H_{i}^{0}$ and, for $m=1, \ldots, k^{a,<\infty}$, let $T_{i}^{a, m}=\left\{t_{i}^{a, m}\right\}$ be the depth- $m$ type that assigns probability 1 to $\theta^{a_{i}}$ and to $T_{-i}^{a, m-1}$. For $m=1, \ldots, k^{a, \infty}$, let $T_{i}^{a, k^{<\infty}+m}$ be a finite set of infinite-depth types that assign probability 1 to $\theta^{a_{i}}$ and to $\bigcup_{\ell=1}^{k^{a,<\infty}+m-1} T_{-i}^{a, \ell}$. 
Let $T_{i}^{a}:=\bigcup_{\ell=0}^{k^{a,<\infty}+k^{a, \infty}} T_{i}^{a, \ell}$. Then $M^{a}:=\left(\widetilde{\Theta}^{a}, T^{a}\right)$ is a finite model. Again, each type in $M^{a}$ has a unique type rank.

Step 3. Defining the model $M$. Let $\widetilde{\Theta}:=\widetilde{\Theta}^{A^{\prime}} \cup \bigcup_{a} \widetilde{\Theta}^{a}$ and $T_{i}:=T_{i}^{A^{\prime}} \cup \bigcup_{a} T_{i}^{a}$. Then $M:=(\widetilde{\Theta}, T)$ is a finite (nonempty) model.

Step 4. Robustness for $M^{A^{\prime}}$. We next show that every strict Bayesian-Nash equilibrium of $M^{A^{\prime}}$ is robust. That is, let $\sigma$ be a strict Bayesian-Nash equilibrium of $M^{A^{\prime}}$. Then we claim that there is $\eta>0$ and $\kappa<\infty$ such that for every $(\eta, \kappa)$ perturbation $\left(M^{\prime}, \tau\right)$ of $M^{A^{\prime}}$, where $M^{\prime}=\left(\widetilde{\Theta}^{\prime}, T^{\prime}\right)$, there is a Bayesian-Nash equilibrium $\sigma^{\prime}$ for $M^{\prime}$ that coincides with $\sigma$ on $\tau\left(T^{A^{\prime}}\right)$.

To show this, let $\sigma$ be a strict Bayesian-Nash equilibrium for $M^{A^{\prime}}$. Since $\sigma$ is a strict Bayesian-Nash equilibrium for $M^{A^{\prime}}$, there is $z>0$ such that for every $i=1,2, t_{i} \in T_{i}^{A^{\prime}}$, and $a_{i}^{\prime} \neq \sigma_{i}\left(t_{i}\right)$,

$$
\int_{\Theta \times T_{-i}^{A^{\prime}}} u_{i}\left(\sigma_{i}\left(t_{i}\right), \sigma_{-i}\left(t_{-i}\right), \theta\right) \mathrm{d} \psi_{t_{i}}-\int_{\Theta \times T_{-i}^{A^{\prime}}} u_{i}\left(\sigma_{i}\left(t_{i}\right), \sigma_{-i}\left(t_{-i}\right), \theta\right) \mathrm{d} \psi_{t_{i}} \geq z
$$

Also note that since $u_{i}$ is a continuous function defined on a compact space, there is $c>0$ such that $u_{i}\left(a_{i}, a_{-i}, \theta\right) \in\left[-\frac{1}{2} c, \frac{1}{2} c\right]$ for all $i, a_{i}, a_{-i}$, and $\theta$.

Let $\kappa=k^{A^{\prime},<\infty}+k^{A^{\prime}, \infty}+1$. Fix $\eta \in(0,1)$ such that the $(\eta, \kappa)$ balls of the types in $M^{A^{\prime}}$ are disjoint (i.e., if $t_{i}$ and $t_{i}^{\prime}$ are distinct types in $T_{i}^{A^{\prime}}$, then $\left.O_{\eta, \kappa}\left(t_{i}\right) \cap O_{\eta, \kappa}\left(t_{i}^{\prime}\right)=\varnothing\right)$. (Such an $\eta$ exists since $M^{A^{\prime}}$ is finite.) Since $\eta<1$, if $t_{i}$ has a finite depth, then any type in $O_{\eta, \kappa}\left(t_{i}\right)$ has the same depth as $t_{i}$ (and thus the same type rank).

Let $\left(M^{\prime}, \tau\right)$, with $M^{\prime}=\left(\widetilde{\Theta}^{\prime}, T^{\prime}\right)$, be an $(\eta, \kappa)$ perturbation of $M^{A^{\prime}}$. We define auxiliary profiles $\sigma^{\prime \prime}$ and $\sigma^{\prime \prime \prime}$. Let $T_{i}^{\prime \prime}$ be the set of types $t_{i}^{\prime} \in T_{i}^{\prime}$ such that there is $t_{i} \in T_{i}^{A^{\prime}}$ such that $t_{i}^{\prime}=\tau_{i}\left(t_{i}\right), t_{i}^{\prime}=t_{i}$, or $t_{i}^{\prime} \in O_{\eta, \kappa}\left(t_{i}\right)$. (Note that these possibilities are not mutually exclusive.) Define the strategy profile $\sigma^{\prime \prime}$ for the types in $T^{\prime \prime}$ as follows. For every $t_{i}^{\prime} \in T_{i}^{\prime}$ such that $t_{i}^{\prime}=\tau_{i}\left(t_{i}\right)$ for some $t_{i} \in T_{i}^{A^{\prime}}$, let $\sigma_{i}^{\prime \prime}\left(t_{i}^{\prime}\right)=\sigma_{i}\left(t_{i}\right)$. Otherwise, if $t_{i}^{\prime} \in T_{i}^{\prime} \cap T_{i}^{A^{\prime}}$, let $\sigma_{i}^{\prime \prime}\left(t_{i}^{\prime}\right)=\sigma_{i}\left(t_{i}^{\prime}\right)$. Otherwise, if $t_{i}^{\prime} \in O_{\eta, \kappa}\left(t_{i}\right)$ for some $t_{i} \in T_{i}^{A^{\prime}}$, then let $\sigma_{i}^{\prime \prime}\left(t_{i}^{\prime}\right)=\sigma_{i}\left(t_{i}\right)$. (By our choice of $\eta$, there is a unique such type $t_{i}$.)

Let $T_{i}^{\prime \prime \prime}:=T_{i}^{\prime} \backslash T_{i}^{\prime \prime}$ and, for $i=1,2$ and $t_{i}^{\prime} \in T_{i}^{\prime \prime \prime}$, let $\sigma_{i}^{\prime \prime \prime}\left(t_{i}^{\prime}\right)$ be a best response to the belief that the opponent plays according to $\sigma_{-i}^{\prime \prime}$ if his type is in $T_{i}^{\prime \prime}$ and according to $\sigma_{-i}^{\prime \prime \prime}$ otherwise. (Such a $\sigma^{\prime \prime \prime}$ exists by standard equilibrium existence arguments.)

Then define the strategy profile $\sigma^{\prime}$ as follows: let $\sigma_{i}^{\prime}\left(t_{i}^{\prime}\right)=\sigma_{i}^{\prime \prime}\left(t_{i}^{\prime}\right)$ if $t_{i}^{\prime} \in T_{i}^{\prime \prime}$ and let $\sigma_{i}^{\prime}\left(t_{i}^{\prime}\right)=\sigma_{i}^{\prime \prime \prime}\left(t_{i}^{\prime}\right)$ otherwise. That is, $\sigma_{i}^{\prime}\left(t_{i}^{\prime}\right)$ is derived from $\sigma_{i}$ if $t_{i}^{\prime}$ is close to a type in $T_{i}$ and is a best response to $\sigma_{-i}^{\prime}$ otherwise. Moreover, since $M^{A^{\prime}}$ is a model and $\sigma$ is a (strict) Bayesian-Nash equilibrium for $M^{A^{\prime}}$, the types in $T \cap T^{\prime}$ also play a best response under $\sigma^{\prime}$. (This follows from a standard "pullback" property; see Friedenberg and Meier 2017.) This means that to check whether $\sigma^{\prime}$ is a Bayesian-Nash equilibrium for $M^{\prime}$, we need only to check the types in $T^{\prime \prime} \backslash T$. These are precisely the types in $T^{\prime}$ that are in the $(\eta, \kappa)$-neighborhoods of the types in $T^{A^{\prime}}$ but not in $T^{A^{\prime}}$ itself.

Consider a type $t_{i}^{\prime} \in T_{i}^{\prime \prime} \backslash T_{i}^{A^{\prime}}$ of type-rank 1 . Then there is $t_{i} \in T_{i}^{A^{\prime}}$ with $\sigma_{i}^{\prime}\left(t_{i}^{\prime}\right)=$ $\sigma_{i}\left(t_{i}\right)$. Moreover, $t_{i}^{\prime}$ assigns probability 1 to $\tau\left(T_{-i}^{A^{\prime}, 0}\right)=T_{-i}^{A^{\prime}, 0}$. By construction, $\sigma_{-i}^{\prime}\left(t_{-i}\right)=$ 
$\sigma_{-i}\left(t_{-i}\right)$ for $t_{-i} \in T_{-i}^{A^{\prime}, 0}$. Also, $t_{i}^{\prime}$ 's belief about $\Theta$ is $\eta$-close to $t_{i}$ 's belief about $\Theta$. Consequently, for every $a_{i} \in A_{i}$,

$$
\left|\int_{\widetilde{\Theta}^{\prime} \times T_{-i}^{\prime}} u_{i}\left(a_{i}, \sigma_{-i}^{\prime}\left(t_{-i}^{\prime}\right), \theta^{\prime}\right) \mathrm{d} \psi_{t_{i}^{\prime}}-\sum_{\widetilde{\Theta}^{A^{\prime} \times T_{-i}^{A^{\prime}}}} u_{i}\left(a_{i}, \sigma_{-i}\left(t_{-i}\right), \theta\right) \mathrm{d} \psi_{t_{i}}\right|<\eta c .
$$

Hence, for every $a_{i} \in A_{i}$,

$$
\int_{\widetilde{\Theta}^{\prime} \times T_{-i}^{\prime}} u_{i}\left(\sigma_{i}^{\prime}\left(t_{i}^{\prime}\right), \sigma_{-i}^{\prime}\left(t_{-i}^{\prime}\right), \theta^{\prime}\right) \mathrm{d} \psi_{t_{i}^{\prime}}-\int_{\widetilde{\Theta}^{\prime} \times T_{-i}^{\prime}} u_{i}\left(a_{i}, \sigma_{-i}^{\prime}\left(t_{-i}^{\prime}\right), \theta^{\prime}\right) \mathrm{d} \psi_{t_{i}^{\prime}} \geq z-2 \eta c .
$$

Next consider a type $t_{i}^{\prime} \in T_{i}^{\prime \prime} \backslash T_{i}^{A^{\prime}}$ of type-rank $m=2, \ldots, k^{A^{\prime},<\infty}$. As before, there is $t_{i} \in T_{i}^{A^{\prime}}$ with $\sigma_{i}^{\prime}\left(t_{i}^{\prime}\right)=\sigma_{i}\left(t_{i}\right)$. Fix $a_{i} \in A_{i}$. Since

$$
\begin{aligned}
& \int_{\widetilde{\Theta}^{\prime} \times T_{-i}^{\prime}} u_{i}\left(a_{i}, \sigma_{-i}^{\prime}\left(t_{-i}^{\prime}\right), \theta^{\prime}\right) \mathrm{d} \psi_{t_{i}^{\prime}} \\
& \quad=\int_{\widetilde{\Theta}^{\prime} \times T_{-i}^{\prime \prime}} u_{i}\left(a_{i}, \sigma_{-i}^{\prime}\left(t_{-i}^{\prime \prime}\right), \theta^{\prime}\right) \mathrm{d} \psi_{t_{i}^{\prime}}+\int_{\widetilde{\Theta}^{\prime} \times T_{-i}^{\prime \prime \prime}} u_{i}\left(a_{i}, \sigma_{-i}^{\prime}\left(t_{-i}^{\prime \prime \prime}\right), \theta^{\prime}\right) \mathrm{d} \psi_{t_{i}^{\prime}},
\end{aligned}
$$

and because for every type $t_{-i}^{\prime \prime} \in T_{-i}^{\prime \prime}$, there is $t_{-i} \in T_{-i}$ with $t_{-i}^{\prime \prime} \in O_{\eta, \kappa}\left(t_{-i}\right)$ and $\sigma_{-i}^{\prime}\left(t_{-i}^{\prime \prime}\right)=$ $\sigma_{-i}\left(t_{-i}\right)$, we have

$$
\left|\int_{\widetilde{\Theta}^{\prime} \times T_{-i}^{\prime}} u_{i}\left(a_{i}, \sigma_{-i}^{\prime}\left(t_{-i}^{\prime}\right), \theta^{\prime}\right) \mathrm{d} \psi_{t_{i}^{\prime}}-\int_{\widetilde{\Theta}^{A^{\prime}} \times T_{-i}^{A^{\prime}}} u_{i}\left(a_{i}, \sigma_{-i}\left(t_{-i}\right), \theta\right) \mathrm{d} \psi_{t_{i}}\right|<(1-\eta) \cdot \eta c+\eta c .
$$

Hence, for every $a_{i} \in A_{i}$,

$$
\int_{\widetilde{\Theta}^{\prime} \times T_{-i}^{\prime}} u_{i}\left(\sigma_{i}^{\prime}\left(t_{i}^{\prime}\right), \sigma_{-i}^{\prime}\left(t_{-i}^{\prime}\right), \theta^{\prime}\right) \mathrm{d} \psi_{t_{i}^{\prime}}-\int_{\widetilde{\Theta}^{\prime} \times T_{-i}^{\prime}} u_{i}\left(a_{i}, \sigma_{-i}^{\prime}\left(t_{-i}^{\prime}\right), \theta^{\prime}\right) \mathrm{d} \psi_{t_{i}^{\prime}} \geq z-2 \eta c \cdot(2-\eta) .
$$

We next consider the $(\eta, \kappa)$ perturbations of the infinite-depth types in $M^{A^{\prime}}$, that is, the types $t_{i}^{\prime} \in T_{i}^{\prime \prime} \backslash T_{i}^{A^{\prime}}$ such that there is $t_{i} \in T_{i}$ with type-rank $m=k^{A^{\prime},<\infty}+1, \ldots, k^{A^{\prime}, \infty}$. Suppose $t_{i}^{\prime}$ is an $(\eta, \kappa)$ perturbation of a type $t_{i} \in T_{i}$ with type-rank $k^{A^{\prime},<\infty}+1$. The critical observation is that while the depth of reasoning of $t_{i}^{\prime}$ can be arbitrarily high (in fact, its depth could be infinite), its belief $\psi_{t_{i}^{\prime}}$ is largely determined by its $\left(k^{A^{\prime},<\infty}+1\right)$ th-order belief: since $t_{i}$ assigns probability 1 at order $k^{A^{\prime},<\infty}+1$ to the opponent having a type in $\bigcup_{\ell=1}^{k^{A^{\prime},<\infty}} T_{-i}^{A^{\prime}, \ell}$ and $t_{i}^{\prime}$ is in the $(\eta, \kappa)$ neighborhood of $t_{i}$ (for $\kappa \geq k^{A^{\prime},<\infty}+1$ ), $t_{i}^{\prime}$ assigns probability $1-\eta$ at order $k^{A^{\prime},<\infty}+1$ to the $(\eta, \kappa)$ neighborhood of $\bigcup_{\ell=1}^{k^{A^{\prime},<\infty}} T_{-i}^{A^{\prime}, \ell}$. So mass $1-\eta$ of $\psi_{t_{i}^{\prime}}$ is determined at order $k^{A^{\prime},<\infty}$. A similar argument applies for infinitedepth types with type-rank $m>k^{A^{\prime},<\infty}+1$ (given that $\kappa=k^{A^{\prime},<\infty}+k^{A^{\prime}, \infty}+1$ ).

Hence, the argument for the infinite-depth case is the same as for the finite-depth case: for every $m=k^{A^{\prime},<\infty}+1, \ldots, k^{A^{\prime}, \infty}$, every type in $t_{i}^{\prime} \in T_{i}^{\prime \prime} \backslash T_{i}^{A^{\prime}}$ that is an $(\eta, \kappa)$ perturbation of a type in $T_{i}^{A^{\prime}}$ with type-rank $m$, and for every $a_{i} \in A_{i}$.

$$
\int_{\widetilde{\Theta}^{\prime} \times T_{-i}^{\prime}} u_{i}\left(\sigma_{i}^{\prime}\left(t_{i}^{\prime}\right), \sigma_{-i}^{\prime}\left(t_{-i}^{\prime}\right), \theta^{\prime}\right) \mathrm{d} \psi_{t_{i}^{\prime}}-\int_{\widetilde{\Theta}^{\prime} \times T_{-i}^{\prime}} u_{i}\left(a_{i}, \sigma_{-i}^{\prime}\left(t_{-i}^{\prime}\right), \theta^{\prime}\right) \mathrm{d} \psi_{t_{i}^{\prime}} \geq z-2 \eta c \cdot(2-\eta) .
$$


To summarize, if $\eta>0$ is sufficiently small so that $z>2 \eta c \cdot(2-\eta)$ and the $(\eta, \kappa)$ balls around the types in $M$ are disjoint, then $\sigma^{\prime}$ is a Bayesian-Nash equilibrium. This conclusion does not depend on the particular $(\eta, \kappa)$ perturbation that we considered: for any $\eta>0$ such that $z>2 \eta c \cdot(2-\eta)$ and the $(\eta, \kappa)$ balls around the types in $M$ are disjoint, any $(\eta, \kappa)$ perturbation has a Bayesian-Nash equilibrium that coincides with $\sigma$ on the image of $M^{A^{\prime}}$. Accordingly, if $z>2 \eta c \cdot(2-\eta)$, then $\sigma$ is $(\eta, \kappa)$-robust.

Step 4. Robustness for $M^{a}, a \in A$. For any nonempty model $M^{a}$, every strict BayesianNash equilibrium of $M^{a}$ is robust. While this can be shown using an argument similar to the one in Step 3, there is a much easier and much more direct proof: every type in $M^{a}$ has a strictly dominant action, so any type whose beliefs about $\Theta$ are $\eta$-close to one of the types in $M^{a}$ has a unique rationalizable action for $\eta>0$ sufficiently small (under the usual weak topology on $\Delta(\Theta)$ ). In this case, the beliefs of types about the opponent's action, or their beliefs about their opponent's belief about their opponent's actions, and so on, are all immaterial.

Step 5. Robustness for $M$. The proof that any strict Bayesian-Nash equilibrium for $M$ is robust is essentially a combination of Steps 3 and 4, and is thus omitted. (It does not immediately follow from the proofs in Steps 3 and 4 in isolation, however. This is because even though $M^{A^{\prime}}$ and $M^{a}$ are disjoint, some of their perturbations may not be (even if $\eta$ is small and $\kappa$ is large): indeed, as perturbed models relax the commonknowledge restrictions embodied in the original model, they tend to be large.)

The model $M$ is consistent with $\left(k^{A^{\prime}, \infty}-1\right)$ th-order belief in an infinite depth. Hence, by choosing $k^{A^{\prime}, \infty}$ appropriately, we obtain a model that is consistent with $m$ thorder belief in an infinite depth for arbitrary depth.

It is easy to extend the construction: the result would also hold if we had added types to $M$ that assign positive probability to $M^{A^{\prime}}$ and to $M^{a}, a \in A$ (assuming the latter is nonempty for some $a$ ), as long as incentives remain strict and the equilibrium actions of types with multiplicity are pinned down by the equilibrium actions of types with a lower level of sophistication (as given by their type-rank).

\section{D.11 Proof of Proposition 8}

Take $\widetilde{\Theta}:=\left\{\theta^{*}, \theta^{\tilde{a}}, \theta^{\tilde{b}}\right\}$, where $\theta^{*}$ is a state at which the complete-information game has two strict Nash equilibria, and $\theta^{\tilde{a}}$ and $\theta^{\tilde{b}}$ are states at which $\tilde{a}$ and $\tilde{b}$ are strictly dominant for both players. (Such states exist by the definition of global games and the assumption of symmetry.)

Define the model $M=(\widetilde{\Theta}, T)$ as in the proof of Proposition 7, except that the types in $T_{i}^{A^{\prime}, m}$ assign probability 1 to $\theta^{*}$ and that we take $T_{a}=\varnothing$ if $a \neq(\tilde{a}, \tilde{a}),(\tilde{b}, \tilde{b})$. Then $M$ is a finite model with complete information.

Define the strategy profile $\sigma^{\tilde{a}}$ as follows. For any type $h_{i}$ in $M^{(\tilde{a}, \tilde{a})}$ (where $\tilde{a}$ is strictly dominant) or in $M^{A^{\prime}}$ (where the complete-information game has two strict Nash equilibria), define $\sigma_{i}^{\tilde{a}}\left(h_{i}\right)=\tilde{a}$. For any type $h_{i}$ in $M^{(\tilde{b}, \tilde{b})}$ (where $\tilde{b}$ is strictly dominant), define $\sigma_{i}^{\tilde{a}}\left(h_{i}\right)=\tilde{b}$. 
Define $\sigma^{\tilde{b}}$ analogously: for any type $h_{i}$ in $M^{(\tilde{b}, \tilde{b})}$ (where $\tilde{b}$ is strictly dominant) or in $M^{A^{\prime}}$ (where the complete-information game has two strict Nash equilibria), define $\sigma_{i}^{\tilde{b}}\left(h_{i}\right)=\tilde{b}$. For any type $h_{i}$ in $M^{(\tilde{a}, \tilde{a})}$ (where $\tilde{a}$ is strictly dominant), define $\sigma_{i}^{\tilde{b}}\left(h_{i}\right)=\tilde{a}$.

It is easy to check that $\sigma^{\tilde{a}}$ and $\sigma^{\tilde{b}}$ are strict Bayesian-Nash equilibria. As such, they are robust (Proposition 7). In particular, the predictions remain valid if we introduce a small amount of information about payoffs.

\section{D.12 Proof of Lemma 7}

Clearly, $R_{i}^{T, 0}\left(t_{i}\right)=R_{i}^{0}\left(h_{i}^{T}\left(t_{i}\right)\right)$. For $m>0$, suppose that for all $n \leq m-1, R_{i}^{T, n}=R_{i}^{n} \circ h_{i}^{T}$. As in the proof of Lemma 12, if $a_{i} \in R_{i}^{m}\left(h_{i}^{T}\left(t_{i}\right)\right)$, then there is a measurable conjecture $s_{-i}: \Theta \times H_{-i} \rightarrow \Delta\left(A_{-i}\right)$ such that $a_{i}$ is a best response for $h_{i}^{T}\left(t_{i}\right)$ under the conjecture $s_{-i}$. Then $s_{-i} \circ h_{-i}^{T}$ is a measurable conjecture such that $a_{i}$ is a best response for $t_{i}$ under the conjecture, so $a_{i} \in R_{i}^{T, m}\left(t_{i}\right)$. Conversely, suppose $a_{i} \in R_{i}^{T, m}\left(t_{i}\right)$. Then there is a belief $\mu_{t_{i}} \in \Delta\left(\Theta \times \operatorname{Gr}\left(R_{-i}^{T, m-1}\right)\right)$ so that $a_{i}$ is a best response to $\mu_{t_{i}}$. The belief $\mu_{t_{i}}$ defines a belief $\mu_{h_{i}} \in \Delta\left(\Theta \times \operatorname{Gr}\left(R_{-i}^{m-1}\right)\right)$ in the obvious way. Then, by the Kuratowski-Ryll-Nardzweski selection theorem, there is a measurable conjecture $s_{-i}: \Theta \times H_{-i} \rightarrow \Delta\left(A_{-i}\right)$ such that $a_{i}$ is a best response against $s_{-i}$ for $h_{i}^{T}\left(t_{i}\right)$, so $a_{i} \in R_{i}^{m}\left(h_{i}^{T}\left(t_{i}\right)\right)$. It is now immediate that $R_{i}^{T, \infty}\left(t_{i}\right)=R_{i}^{\infty}\left(h_{i}^{T}\left(t_{i}\right)\right)$.

\section{D.13 Proof of Lemma 8}

The proof follows from a number of lemmas.

Lemma 13. For $i=1,2$ and $k \in \mathbb{N}, \widetilde{\Omega}_{i}^{k}, \Omega_{i}^{k}, \widetilde{H}_{i}^{k}$, and $H_{i}^{k}$ are compact metric.

Proof. The proof is by induction. Clearly, $\widetilde{H}_{i}^{0}$ and $H_{i}^{0}$ are compact metric, so that $\widetilde{\Omega}_{i}^{0}$, $\Omega_{i}^{0}, \widetilde{H}_{i}^{1}$, and $H_{i}^{1}$ are also compact metric. Suppose $\widetilde{\Omega}_{i}^{\ell}, \Omega_{i}^{\ell}, \widetilde{H}_{i}^{\ell+1}$, and $H_{i}^{\ell+1}$ are compact metric for each $i=1,2$ and $\ell \leq k-1$. Then $\widetilde{\Omega}_{i}^{k}$ and $\Omega_{i}^{k}$ are compact metric. It remains to show that $\widetilde{H}_{i}^{k+1}$ and $H_{i}^{k+1}$ are compact metric. As $\Delta\left(\widetilde{\Omega}_{i}^{k}\right)$ and $\Delta\left(\Omega_{i}^{k}\right)$ are compact metric, we need to show that $\widetilde{H}_{i}^{k+1}$ and $H_{i}^{k+1}$ are a closed subset of $\widetilde{H}_{i}^{k} \times \Delta\left(\widetilde{\Omega}_{i}^{k}\right)$ and $\widetilde{H}_{i}^{k} \times \Delta\left(\Omega_{i}^{k}\right)$, respectively. We prove the claim for $\widetilde{H}_{i}^{k+1}$; the proof for $H_{i}^{k+1}$ is similar. Let $h_{i}=\left(x_{i}, \mu_{i}^{0}, \ldots, \mu_{i}^{k+1}\right) \in \widetilde{H}_{i}^{k} \times \Delta\left(\widetilde{\Omega}_{i}^{k}\right)$ and suppose there is a sequence $\left(h_{i}^{n}\right)_{n \in \mathbb{N}}$ in $\widetilde{H}_{i}^{k+1}$, where $h_{i}^{n}=\left(x_{i}^{n}, \mu_{i}^{0, n}, \mu_{i}^{2, n}, \ldots, \mu_{i}^{k+1, n}\right)$, such that $h_{i}^{n} \rightarrow h_{i}$. It is sufficient to show that $h_{i} \in \widetilde{H}_{i}^{k}$. If we show that

$$
\operatorname{marg}_{\widetilde{\Omega}_{i}^{k-1}} \mu_{i}^{k+1, n} \rightarrow \operatorname{marg}_{\widetilde{\Omega}_{i}^{k-1}} \mu_{i}^{k+1}
$$

and

$$
\mu_{i}^{k, n} \rightarrow \mu_{i}^{k},
$$

the proof is complete: Because $h_{i}^{n} \in \widetilde{H}_{i}^{k+1}$ for all $n$, it follows that

$$
\operatorname{marg} \widetilde{\Omega}_{i}^{k-1} \mu_{i}^{k+1}=\mu_{i}^{k},
$$


so that $h_{i} \in \widetilde{H}_{i}^{k+1}$. But using that $\widetilde{H}_{i}^{k} \times \Delta\left(\widetilde{\Omega}_{i}^{k}\right)$ is endowed with the product topology, (D.1) and (D.2) follow immediately from the assumption that $h_{i}^{n} \rightarrow h_{i}$.

Lemma 14 (Heifetz 1993, Theorem 6). For any $\left(x_{i}, \mu_{i}^{0}, \ldots, \mu_{i}^{k}\right) \in \widetilde{H}_{i}^{k}$, there exists $\mu_{i}^{k+1} \in$ $\Delta\left(\widetilde{\Omega}_{i}^{k}\right)$ such that $\left(x_{i}, \mu_{i}^{0}, \ldots, \mu_{i}^{k}, \mu_{i}^{k+1}\right) \in \widetilde{H}_{i}^{k+1}$.

The proof is similar to the proof of Theorem 6 of Heifetz (1993) and thus is omitted. We are now ready to prove Lemma 8 . By Lemma $14, \widetilde{H}_{i}^{k}$ is nonempty. Also, the projection function from $\widetilde{H}_{i}^{k}$ into $\widetilde{H}_{i}^{k-1}$ is surjective. By standard arguments, the inverse limit space $H_{i}^{\infty}$ is nonempty. Since $H_{i}^{\infty}$ is a closed subset of the compact metric space $\widetilde{H}_{i}^{0} \times \prod_{k=0}^{\infty} \Delta\left(\Omega_{i}^{k}\right)$, it is compact metric. Finally, $H_{i}$ is Polish since it is the disjoint union of a countable family of compact metric (and thus Polish) spaces.

\section{D.14 Proof of Lemma 9}

We first prove the first claim. By Lemma 8 , the space $\Theta \times H_{-i}^{\infty}$ is a nonempty Polish space for every player $i$. By a version of the Kolmogorov consistency theorem, for each belief hierarchy $h_{i}^{\infty}=\left(x_{i}, \mu_{i}^{0}, \mu_{i}^{1}, \ldots\right) \in H_{i}^{\infty}$ of infinite depth, there exists a unique Borel probability measure $\mu_{i}^{\infty}$ on $\Theta \times H_{-i}$ such that

$$
\operatorname{marg} \widetilde{\Omega}_{i}^{k} \mu_{i}^{\infty}=\mu_{i}^{k+1}
$$

for all $k$, i.e., the mapping is canonical. The last claim follows immediately by associating the belief $\mu_{i}^{k}$ to the finite hierarchy $h_{i}^{k}=\left(x_{i}, \mu_{i}^{0}, \ldots, \mu_{i}^{k-1}, \mu_{i}^{k}\right) \in \widetilde{H}_{i}^{k}$.

\section{D.15 Proof of Proposition 9}

First consider the infinite-depth hierarchies. Lemma 9 shows that each infinite belief hierarchy $h_{i}^{\infty}=\left(x_{i}, \mu_{i}^{0}, \mu_{i}^{1}, \ldots\right) \in H_{i}^{\infty}$ corresponds to a unique Borel probability measure on $\Theta \times H_{-i}$, and the mapping is canonical. Moreover, the signal $x_{i}$ associated with $h_{i}^{\infty}$ is obtained by projecting $h_{i}^{\infty}$ onto $X_{i}$. Denote the function that maps $H_{i}^{\infty}$ into $X_{i} \times \Delta\left(\Theta \times H_{-i}\right)$ in this way by $\tilde{\psi}_{i}^{\infty}$. Conversely, let $r_{i}^{\infty}: X_{i} \times \Delta\left(\Theta \times H_{-i}\right) \rightarrow$ $H_{i}^{\infty}$ be the mapping that assigns to each $\left(x_{i}, \mu_{i}\right) \in X_{i} \times \Delta\left(\Theta \times H_{-i}\right)$ the hierarchy $\left(x_{i}, \operatorname{marg}_{\Theta} \mu_{i}, \operatorname{marg}_{\widetilde{\Omega}_{i}^{0}} \mu_{i}, \operatorname{marg}_{\widetilde{\Omega}_{i}^{1}} \mu_{i}, \ldots\right) \in X_{i} \times \Delta(\Theta) \times \prod_{k \geq 0} \Delta\left(\widetilde{\Omega}_{i}^{k}\right)$. The function $r_{i}^{\infty}$ is the inverse of $\tilde{\psi}_{i}^{\infty}$; it remains to show that $\tilde{\psi}_{i}^{\infty}$ and $r_{i}^{\infty}$ are continuous. The function $\tilde{\psi}_{i}^{\infty}$ is continuous if and only if $h_{i}^{n} \rightarrow h_{i}$ in $H_{i}^{\infty}$ implies $\psi_{i}^{\infty}\left(h_{i}^{n}\right) \rightarrow \psi_{i}^{\infty}\left(h_{i}\right)$ in $X_{i} \times \Delta\left(\Theta \times H_{-i}\right)$. This follows from the continuity of the projection function and the fact that the cylinders form a convergence-determining class in $\Theta \times H_{-i}$, with the value of $\tilde{\psi}_{i}^{\infty}\left(h_{i}\right)$ for $h_{i}=\left(x_{i}, \mu_{i}^{0}, \mu_{i}^{1}, \ldots\right)$ on the cylinders being given by the $\mu_{i}^{k}$ s. Finally, it follows from the continuity of the identity function and the marginal operator that $r_{i}^{\infty}$ is continuous.

For the case of finite-depth hierarchies, simply set $\psi_{i}^{k}\left(h_{i}^{k}\right):=\left(x_{i}, \mu_{i}^{k}\right)$ for each $h_{i}^{k}=$ $\left(x_{i}, \mu_{i}^{0}, \ldots, \mu_{i}^{k-1}, \mu_{i}^{k}\right) \in \widetilde{H}_{i}^{k}$. Continuity of the mapping $\psi_{i}^{k}$ is immediate. 


\section{REFERENCES}

Aliprantis, Charalambos D. and Kim C. Border (2006), Infinite Dimensional Analysis: A Hitchhiker's Guide, third edition. Springer, Berlin. [447, 449, 450, 451, 452]

Bergemann, Dirk and Stephen Morris (2005), "Robust mechanism design.” Econometrica, 73, 1771-1813. [429]

Billingsley, Patrick (1968), Convergence of Probability Measures. Wiley, New York. [451, $453,455]$

Brandenburger, Adam (2003), “On the existence of a 'complete' possibility structure.” In Cognitive Processes and Economic Behavior (Marcello Basili, Nicola Dimitri, and Itzhak Gilboa, eds.), Routledge Siena Studies in Political Economy, 30-34, Routledge, London. [446]

Carlsson, Hans and Eric van Damme (1993), "Global games and equilibrium selection." Econometrica, 61, 989-1018. [420, 421, 422, 424, 426, 438, 439, 442]

Chen, Yi-Chun (2012), "A structure theorem for rationalizability in the normal form of dynamic games." Games and Economic Behavior, 75, 587-597. [417]

Chen, Yi-Chun, Alfredo Di Tillio, Eduardo Faingold, and Siyang Xiong (2010), "Uniform topologies on types." Theoretical Economics, 5, 445-478. [417]

Chen, Yi-Chun, Alfredo Di Tillio, Eduardo Faingold, and Siyang Xiong (2017), "Characterizing the strategic impact of misspecified beliefs." Review of Economic Studies, 84, 1424-1471. [417]

Chen, Yi-Chun, Satoru Takahashi, and Siyang Xiong (2014a), "The Weinstein-Yildiz selection and robust predictions with arbitrary payoff uncertainty.” Unpublished paper, University of Bristol. [417]

Chen, Yi-Chun, Satoru Takahashi, and Siyang Xiong (2014b), "The robust selection of rationalizability." Journal of Economic Theory, 151, 448-475. [417]

Crawford, Vincent P., Miguel A. Costa-Gomes, and Nagore Iriberri (2013), "Structural models of nonequilibrium strategic thinking: Theory, evidence, and applications." Journal of Economic Literature, 51, 5-62. [416, 419, 425, 444]

Dekel, Eddie, Drew Fudenberg, and Stephen Morris (2006), “Topologies on types.” Theoretical Economics, 1, 275-309. [417]

Dekel, Eddie, Drew Fudenberg, and Stephen Morris (2007), "Interim correlated rationalizability.” Theoretical Economics, 2, 15-40. [419, 423, 433, 434, 445, 447, 448]

Dekel, Eddie and Marciano Siniscalchi (2015), “Epistemic game theory.” In Handbook of Game Theory With Economic Applications, Vol. 4 (Petyon Young and Shmuel Zamir, eds.), 619-702, North-Holland, Amsterdam. [430]

Friedenberg, Amanda and Martin Meier (2017), “The context of the game." Economic Theory, 63, 347-386. [458] 
Harsanyi, John (1967), "Games on incomplete information played by Bayesian players. Part I.” Management Science, 14, 159-182. [418, 444]

Heifetz, Aviad (1993), "The Bayesian formulation of incomplete information-The noncompact case." International Journal of Game Theory, 21, 329-338. [462]

Heifetz, Aviad and Willemien Kets (2012), "All types naive and canny.” Discussion Paper 1550, Center for Mathematical Studies in Economics and Management Science, Northwestern University. [415]

Heifetz, Aviad, Martin Meier, and Burkhard C. Schipper (2013), "Unawareness, beliefs and speculative trade." Games and Economic Behavior, 77, 100-121. [446]

Kets, Willemien (2012), “Bounded reasoning and higher-order uncertainty." Unpublished paper, Northwestern University. [444]

Kets, Willemien (2013), "Finite depth of reasoning and equilibrium play in games with incomplete information.” Discussion paper 1569, Center for Mathematical Studies in Economics and Management Science, Northwestern University. [444]

Kreps, David M., Paul Milgrom, John Roberts, and Robert Wilson (1982), "Rational cooperation in the finitely repeated prisoners' dilemma." Journal of Economic Theory, 27, 245-252. [417, 443]

Kreps, David M. and Robert Wilson (1982), "Reputation and imperfect information.” Journal of Economic Theory, 27, 253-279. [417, 443]

Lipman, Barton L. (1994), "A note on the implications of common knowledge of rationality." Games and Economic Behavior, 6, 114-129. [447]

Lubin, Arthur (1974), "Extensions of measures and the von Neumann selection theorem." Proceedings of the American Mathematical Society, 43, 118-122. [449]

Mertens, Jean-François and Shmuel Zamir (1985), "Formulation of Bayesian analysis for games with incomplete information." International Journal of Game Theory, 14, 1-29. [418, 431, 432, 444, 445]

Milgrom, Paul and John Roberts (1982), "Predation, reputation and entry deterrence." Journal of Economic Theory, 27, 280-312. [417, 443]

Morris, Stephen and Hyun Song Shin (2003), "Global games: Theory and applications.” In Advances in Economics and Econometrics, Vol. 1 (Mathias Dewatripont, Lars Peter Hansen, and Stephen J. Turnvosky, eds.), 56-114, Cambridge University Press, Cambridge. [424]

Murayama, Kota (2015), "Robust predictions under finite depth of reasoning." Unpublished paper, Northwestern University. [444]

Penta, Antonio (2012), "Higher order uncertainty and information: Static and dynamic games.” Econometrica, 80, 631-660. [417]

Penta, Antonio (2013), "On the structure of rationalizability for arbitrary spaces of uncertainty." Theoretical Economics, 8, 405-430. [417, 428] 
Rubinstein, Ariel (1989), "The electronic mail game: Strategic behavior under 'almost common knowledge'.” American Economic Review, 79, 385-391. [417, 421, 424, 444]

Strzalecki, Tomasz (2014), "Depth of reasoning and higher order beliefs.” Journal of Economic Behavior \& Organization, 108, 108-122. [417, 424, 444]

Weinstein, Jonathan and Muhamet Yildiz (2007), "A structure theorem for rationalizability with application to robust predictions of refinements." Econometrica, 75, 365-400. [415, 416, 417, 420, 421, 424, 426, 428, 434, 437, 441, 457]

Weinstein, Jonathan and Muhamet Yildiz (2013), "Robust predictions in infinite-horizon games-an unrefinable folk theorem." Review of Economic Studies, 80, 365-394. [417]

Yildiz, Muhamet (2004), “Generic uniqueness of rationalizable actions.” Unpublished paper, MIT. [448]

Co-editor George J. Mailath handled this manuscript.

Manuscript received 11 February, 2015; final version accepted 10 March, 2017; available online 20 March, 2017. 